\title{
The Population of Weak Mg II Absorbers I. A Survey of 26 QSO HIRES/Keck Spectra ${ }^{1}$
}

\author{
Christopher W. Churchill², Jane R. Rigby, Jane C. Charlton ${ }^{3}$ \\ Department of Astronomy and Astrophysics \\ The Pennsylvania State University, University Park PA 16802 \\ cwc@astro.psu.edu, jrigby@astro.psu.edu,charlton@astro.psu.edu
}

Steven S. Vogt ${ }^{2,4}$

Board of Astronomy and Astrophysics

University of California, Santa Cruz CA 95064

vogt@ucolick.org

\begin{abstract}
We present a search for "weak" MgII absorbers [those with $W_{\mathrm{r}}(2796) \leq 0.3 \AA$ ] in the HIRES/Keck spectra of 26 QSOs. We found 30, of which 23 are newly discovered. The spectra are $80 \%$ complete to $W_{\mathrm{r}}(2796)=0.02 \AA$ and have a cumulative redshift path of $\sim 17.2$ for the redshift range $0.4 \leq z \leq$ 1.4. The number of absorbers per unit redshift, $d N / d z$, is seen to increase as the equivalent width threshold is decreased; we obtained $d N / d z=1.74 \pm 0.10$ for our $0.02 \leq W_{\mathrm{r}}(2796) \leq 0.3 \AA$ sample. The equivalent width distribution follows a power law, $N(W) \propto W^{-\delta}$, with $\delta \sim 1.0$; there is no turnover down to $W_{\mathrm{r}}(2796)=0.02 \AA$ at $\langle z\rangle=0.9$. Thus, weak absorbers comprise at least $65 \%$ of the total MgII absorption population, which outnumbers Lyman limit systems (LLS) by a factor of $3.8 \pm 1.1$; the majority of weak MgII absorbers must arise in sub-LLS environments. Tentatively, we predict that $\sim 5 \%$ of the $\operatorname{Ly} \alpha$ forest clouds with $W_{\mathrm{r}}(\operatorname{Ly} \alpha) \geq 0.1 \AA$ will have detectable $\operatorname{Mg}$ II absorption to $W_{\mathrm{r}}^{\min }(2796)=0.02 \AA$ and that this is primarily a high-metallicity selection effect $\left(\left[Z / Z_{\odot}\right] \geq-1\right)$. The individual absorbing "clouds" have $W_{\mathrm{r}}(2796) \leq 0.2 \AA$ and their narrow (often unresolved) line widths imply temperatures of $\sim 25,000 \mathrm{~K}$. These facts imply that Mg II absorbing structures figure prominently as tracers of sub-LLS environments where gas has been processed by stars. We compare the number density of MgII systems with that of both high and low surface brightness galaxies and examine the range of sizes inferred for MgII absorbers with $W_{\mathrm{r}}(2796) \geq 0.02 \AA$. We discuss evidence for a range of ionization conditions (low, high, and multi-phase) in the population of systems selected by weak Mg II absorption and speculate as to the expected nature and redshift evolution of this population.
\end{abstract}

Subject headings: galaxies: interstellar medium — galaxies: evolution — quasars: absorption lines

Submitted to $A p J$

\footnotetext{
${ }^{1}$ Based in part on observations obtained at the W. M. Keck Observatory, which is jointly operated by the University of California and the California Institute of Technology.

${ }^{2}$ Visiting Astronomer at the W. M. Keck Observatory

${ }^{3}$ Center for Gravitational Physics and Geometry, Pennsylvania State University

${ }^{4} \mathrm{UCO} /$ Lick Observatories, University of California
} 


\section{Introduction}

We present a survey for "weak" MgII $\lambda \lambda 2976,2803$ absorption in the spectra of QSOs obtained with the HIRES spectrograph (Vogt et al. 1994) on the Keck I telescope. There have been several Mg II surveys over the previous decade (Lanzetta, Turnshek, \& Wolfe 1987, hereafter LTW; Tytler et al. 1987, hereafter TBSYK; Caulet 1989; Petitjean \& Bergeron 1990, hereafter PB90; Sargent, Steidel, \& Boksenberg 1988, hereafter SSB; Steidel \& Sargent 1992, hereafter SS92). These surveys were complete to a rest-frame MgII $\lambda 2796$ equivalent width, $W_{\mathrm{r}}(2796)$, of $0.3 \AA$ and above. The more comprehensive work of SS92 yielded solid statistics on the equivalent width distribution, redshift number density, large scale velocity clustering, and evolution over the redshift range $0.3 \leq z \leq 2.2$, for which the Mg II doublet can be observed with ground based telescopes.

The shape of the $W_{\mathrm{r}}(2796)$ distribution function at smaller equivalent widths has important implications for our understanding of cosmic chemical evolution and its connection to star producing environments. At $0.3 \leq z \leq 1.0, \mathrm{Mg}$ II absorption with $W_{\mathrm{r}}(2796) \geq 0.3 \AA \mathrm{Mg}$ II has been found to arise within $\sim 40 h^{-1} \mathrm{kpd}$ 同 of normal galaxies (Bergeron \& Boisse 1991: Steidel, Dickinson, \& Persson 1994). These galaxies exhibit a wide range of colors from late-type spirals to the reddest ellipticals (though more luminous galaxies are redder), and have $L_{B}$ and $L_{K}$ luminosity functions consistent with the local luminosity function (types later than Sd are absent). It has been concluded that a wide range of morphological types are contributing to the Mg II absorbing gas cross section, except that isolated low-mass $\left(L_{K} \leq 0.06 L_{K}^{*}\right)$ "faint blue galaxies" are not Steidel, Dickinson, \& Persson 1994. A rapid cut off in the equivalent width distribution would imply that this observed sample of galaxies provides a complete picture of the star forming environments that give rise to $\mathrm{Mg}$ II absorbing gas. Currently, that picture is one in which the galaxy population selected by Mg II absorption is stable, showing very little cosmological evolution from $z \sim 1$.

If, on the other hand, the number of MgII absorbers per unit redshift continues to rise for decreasing $W_{\mathrm{r}}(2796)$, it would be implied that surveys complete to $0.3 \AA$ have unveiled only a small portion of the population of metal-line systems selected by Mg II ab-

\footnotetext{
${ }^{5}$ Throughout this paper we use $q_{0}=0.05$ and express physical sizes in terms of $h=H_{0} / 100$.
}

sorption. Churchill \& Le Brun (1998) have discussed this possibility based upon the discovery of two nearsolar to super-solar metallicity MgII absorbers in the Ly $\alpha$ forest of PKS $0454+039$. As such, our current picture of the relationship between MgII absorbing gas and star forming environments may require some modification. Perhaps the observed $L_{K} \sim 0.06 L_{K}^{*}$ cut off in the luminosity function of MgII absorptionselected galaxies or the cut off at $\sim 40 h^{-1} \mathrm{kpc}$ of MgII absorption around these galaxies does not apply for $W_{\mathrm{r}}(2796) \leq 0.3 \AA$. Alternatively perhaps another population of star forming objects that preferentially give rise to weaker $\mathrm{Mg} I \mathrm{I}$ absorption is implied. It may (or may not) be that this hypothetical alternative population exhibits stochastic cosmological evolution, that the objects do not steadily form stars or maintain stable absorbing gas structures. Or, perhaps both a slight modification to the current picture of MgII absorbing galaxies and the incorporation of another population of objects would be implied. Measuring the statistical absorption properties of the weakest Mg II absorbers is a first step toward verifying or casting new light on such speculations.

Below $W_{\mathrm{r}}(2796) \leq 0.3 \AA$, BP90 and SS92 inferred a cut off in the MgII equivalent width distribution, the number of absorbers per unit redshift per unit equivalent width. However, the completeness of their data below $0.3 \AA$ dropped rapidly. Their conclusions have necessarily been based upon a comparison of the number of Mg II absorbers actually detected with $W_{\mathrm{r}}(2796) \leq 0.3 \AA$, corrected for completeness, to the number of these absorbers predicted by extrapolating the equivalent width distribution. Measurements of the equivalent width distribution, however, were somewhat inconclusive; the data were adequately described either by a power law distribution with slope $\delta=-2.0$ (TBSYK) or $\delta=-1.6$ (SS92), or by an exponential with a characteristic equivalent width of $W_{\mathrm{r}}(2796)=0.66 \AA$ (SS92; LTW). Womble (1995) and Tripp, Lu, \& Savage (1997) have tentatively concluded that the equivalent width distribution continues to rise below $0.3 \AA$. The HIRES/Keck spectra obtained for the thesis of Churchill (1997a; also see Churchill, Vogt, \& Charlton 1998) provide duplicate redshift coverage of 26 of the 103 QSO sight lines studied by SS92. The spectra are $80 \%$ complete to $W_{\mathrm{r}}^{\min }(2796)=0.02 \AA$, and provide an opportunity to directly investigate if in fact there is a paucity of MgII absorbers with $W_{\mathrm{r}}(2796) \leq 0.3 \AA$ and to measure the shape of the distribution of equivalent widths 
well below $0.3 \AA$.

Using a database of high quality high resolution spectra, we have set out to measure the statistical absorption properties of the weakest Mg II systems. The acquisition of the data and their reduction and analysis are described in $\$ 2$. In $\$ 3$, we present our adopted sample of weak Mg II systems. In 8 国 we compute the redshift path density, determine the shape of the equivalent width distribution, examine the clustering, and discuss the general absorption properties of weak MgII absorbers. We discuss the ionization conditions and some inferences about the luminous objects associated with these absorber in $\$$. Our main results are summarized in 8 . 6 .

\section{Observations and Data Analysis}

\subsection{The Sample of QSO Spectra}

A full description of the data acquisition is given in Churchill (1997a) and in Churchill, Vogt, \& Charlton (1998, hereafter CVC98). In short, 26 QSO spectra were obtained with the HIRES spectrometer Vogt et al. 1994) on the Keck I telescope. The QSOs and their emission redshifts, the UT date of the observations, the summed exposure times, and the approximate wavelength coverage are presented in Table 1 .

These spectra were originally part of a targeted survey to study the kinematics of known MgII absorbers compiled by SS92. Thus, each QSO sight line was carefully selected based upon the known presence of one or more MgII absorbers. In fact, sight lines with the largest equivalent width systems were preferentially selected. These "strong" systems were expected to reveal rich kinematic structures because of the observed correlation of the number of components with equivalent width (PB90). As such, the spectra comprise a biased sample of QSO sight lines with "strong" MgII absorbers.

The SS92 survey had a fairly uniform Mg II $\lambda 2796$ rest-frame equivalent width limit of $0.3 \AA$, where the $5 \sigma$ limit has been quoted. Therefore, the lines of sight presented by CVC98 are actually unbiased for MgII systems that have $W_{\mathrm{r}}(2796) \leq 0.3 \AA$, since nothing is known a priori about the presence of these "weak" Mg II systems ${ }^{6}$. However, it is possible that weak systems are preferentially found in sight lines along

\footnotetext{
${ }^{6}$ From this point forward, we refer to "weak" systems as those having $W_{\mathrm{r}}(2796) \leq 0.3 \AA$ and call $W_{\mathrm{r}}(2796)>0.3 \AA$ systems "strong".
}

which strong systems are present, in which case these QSO spectra would not comprise an unbiased sample. We return to this point in $\$ 4.3$.

Though the sample of spectra are well suited for an unbiased survey, they are not ideally suited. Each spectrum was obtained using a single setting of the HIRES echelle and cross disperser. Each has breaks in wavelength coverage redward of $5100 \AA$ because a single setting of the 2048X2048 CCD cannot capture the complete free spectral range beyond this wavelength. The gaps in redshift coverage begin at $z \sim 0.83$ and become more pronounced toward higher redshifts (see Figure 10 c). As discussed in $\$ 2.4$, we searched the spectra for the presence of both members of the doublet; both members had to be present in order for the system to be included in the survey.

\subsection{Data Reduction and Analysis}

The raw data frames were bias corrected, flat fielded, cosmic ray cleaned, combined, and scattered light corrected, using the standard IRAF packages following the techniques described in Churchill (1995) and CVC98. The individual spectra were extracted using the optimal extraction algorithms provided in the noao.twodspec.apextract package. The wavelength calibration was done interactively using the ecidentify task of the noao.imred.echelle package. We have not resampled the data by linearizing the wavelength as a function of pixel along the dispersion direction. Linearizing the data involves a flux conserving interpolation between adjacent pixels. This interpolation introduces correlated errors that are difficult to treat and modifies subtle shapes in absorption profiles when the signal-to-noise ratio is high.

Each full spectrum was then converted into an ASCII file and the remainder of the analysis was performed using our own software (further details are presented in Churchill 1997a). Because of the HIRES echelle format and the large size of the CCD, there is redundant wavelength coverage (at the order ends) below $5100 \AA$. There are well documented techniques available in IRAF for combining these regions using interpolation to resample and combine the data into a "single" spectrum. We have chosen to not resample and combine the regions of redundant coverage. The continuum fitting is a critical step in the analysis

\footnotetext{
${ }^{7}$ IRAF is distributed by the National Optical Astronomy Observatories, which are operated by AURA, Inc., under contract to the NSF.
} 
because it results in correlated errors between pixels. A search for weak features could easily be biased by systematics in the continuum fit. Thus, we took great care with the fitting. The adopted continuum fits, based upon the formalism of Sembach \& Savage (1992), were obtained by minimizing $\chi^{2}$ between the flux values and a smooth function, usually a Legendre polynomial.

During the extraction, the uncertainty in the flux in each pixel was computed based upon a simple variance model that accounted for Poisson noise and read noise. The uncertainty spectra are critical, as we shall discuss below, for the objective identification of absorption features, especially those that are unresolved. If the values in the uncertainty spectrum are smaller than the noise level of the nearby continuum, then too many absorption (and emission) features would be objectively identified to the desired significance level; if the uncertainty spectrum values are larger than the noise in the data, then weak but real absorption features would not be found.

While the resulting uncertainty spectra accurately depicted the relative pixel to pixel uncertainties, we found that their values were often not a good description of the noise level in the continuum. Thus, for each echelle order, we enforced a unity $\chi_{\nu}^{2}$ between the smooth fitted continuum model and the spectrum by scaling the uncertainty spectrum by a single "optimizing" multiplicative factor, $f$. To obtain $f$, we root solved the function $1-\chi_{\nu}^{2}$, where $\chi_{\nu}^{2}=V_{\text {fit }} / f V_{\sigma}$, and $V_{\text {fit }}$ and $V_{\sigma}$ are the variance in the continuum fit and the uncertainty spectrum, respectively. As a result, the uncertainty spectrum retained its overall shape and pixel to pixel structure, but the pixel values more accurately reflected the noise level of the continuum flux values. The process required an iterative convergence algorithm because it was critical to objectively mask absorption features that would otherwise bias the optimizing scaling factor (only continuum pixels were used for the root solving in each iteration).

For the objective identification of unresolved absorption (and emission) features, we have used the formalism presented by Schneider et al. (1993), who developed a technique to compute both an equivalent width spectrum and an equivalent width uncertainty spectrum based upon optimal pixel by pixel weighting with the instrumental spread function. The equivalent width uncertainty spectra provide the rest-frame limiting equivalent width of the Mg II $\lambda 2796$ transition as a function of redshift. The $5 \sigma$ rest-frame sensitiv- ity limits for detecting a $\lambda 2796$ transition as a function of redshift, are shown in Figure 1010 . Where there is no redshift coverage (interorder gaps), we have arbitrarily set the limiting equivalent width to zero.

\subsection{Notes on the Reduction of Selected QSOs}

2.3.1. $Q 0002+051 ; z_{\mathrm{em}}=1.899$

Only a single HIRES spectrum on this QSO was obtained so that removing cosmic rays, especially from the sky, was very problematic. As a consequence, the zero level of the spectrum is uncertain by $\sim 10 \%$ and the measured equivalent widths may be biased by this probable zero-point offset, which varied from echelle order to echelle order. This uncertainty has not been included in the error estimate of the quoted equivalent widths.

\subsection{2. $Q 1548+092 ; z_{\mathrm{em}}=2.749$}

The region of the QSO spectrum we observed is blueward of the CIV emission line by $\sim 100 \AA$. The Ly $\alpha$ forest compromises all transitions of metal line systems that lie blueward of $\sim 4560 \AA$. Thus, we have not searched for $\mathrm{Mg}$ II doublets in the spectrum below this wavelength; we could not confidently detect Mg II systems below $z \sim 0.62$. Observations of this QSO were plagued by 1-2 magnitudes of extinction due to cirrus cloud cover. As such, the signal-to-noise ratio, and thus detection sensitivity, is very low.

\subsection{Doublet Searching}

We have searched for redshifted MgII doublets in our 26 QSO spectra. The sensitivity of the search is not uniform. This is due to the varying observing conditions, the range in the brightness of the observed QSOs, and the wavelength (redshift) dependent sensitivity of the HIRES spectrograph. We will return to the issue of non-uniform sensitivity in $\S 3.3$ and in \$1.

The search for MgII doublets involved the following steps. First, a complete list of features were objectively defined in each QSO spectrum. All features had a $5 \sigma$ detection significance level. To locate candidate Mg II doublets, we then tested the features one by one, starting at the smallest wavelength feature and moving toward larger wavelengths. We assumed each feature was a candidate $\lambda 2796$ line with observed central wavelength $\lambda_{27}$. Then, the expected location of the $\lambda 2803$ feature was computed from 


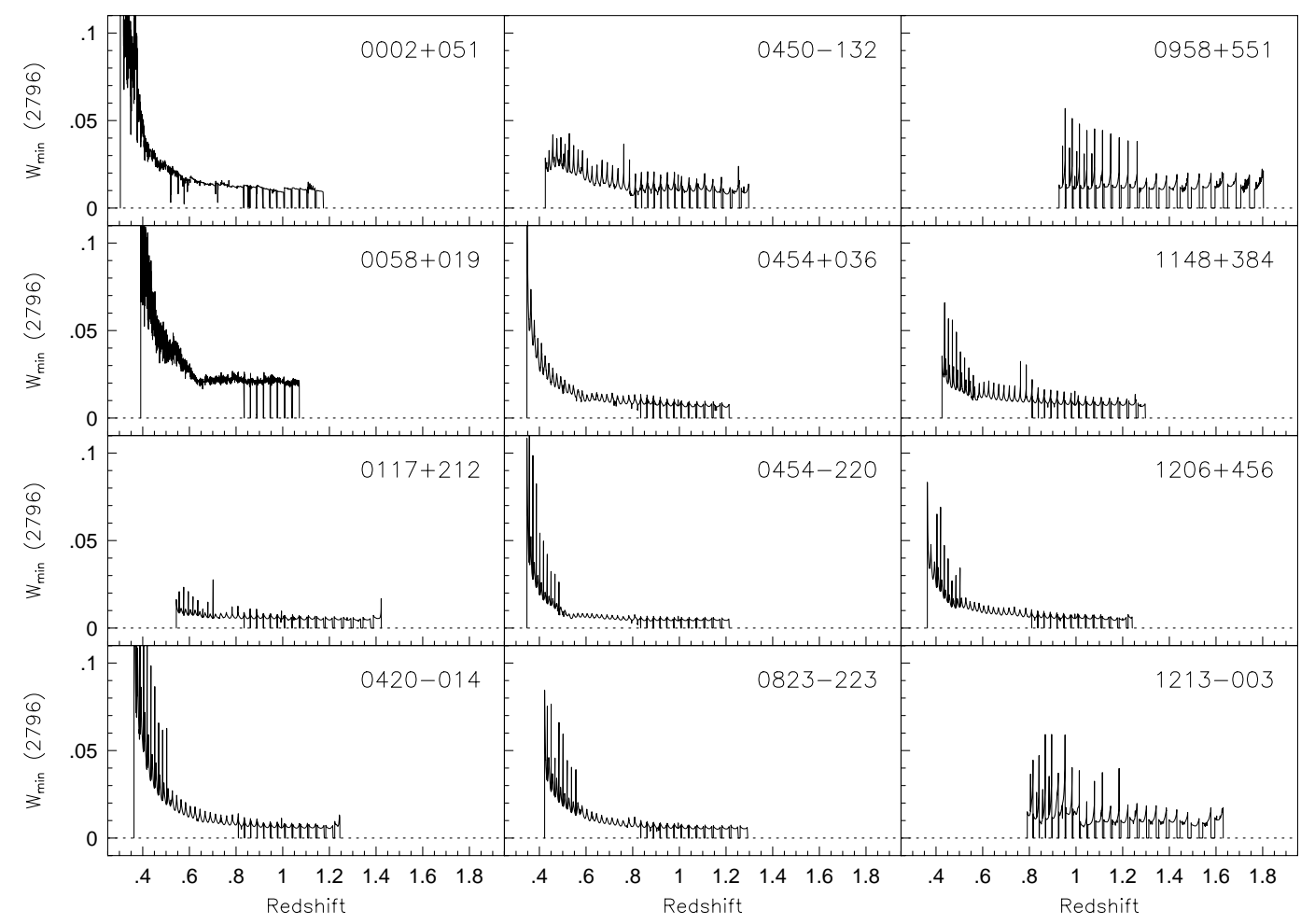

Fig. 1a.- The survey sensitivity, given as the $5 \sigma W_{\mathrm{r}}^{\min }(2796)$, is shown as a function of redshift. Breaks in the echelle spectral coverage, where there is no possibility of detecting a MgII doublet in a given redshift range, are arbitrarily assigned $W_{\mathrm{r}}^{\min }(2796)=0$.

$\lambda_{28}=2803.531\left(\lambda_{27} / 2796.352\right)$. Centered about $\lambda_{28}$, an equivalent width and its uncertainty were measured in an aperture with the same full width at the continuum as that of the candidate $\lambda 2796$ feature. These quantities were measured using the formalism of Sembach \& Savage (1992). The pair was designated as a candidate doublet when the $\lambda 2803$ detection significance was roughly equal to or greater than half that of the $\lambda 2796$ feature (given by the ratio of the $f \lambda$ ) such that the doublet ratio was consistent with $1 \leq \mathrm{DR} \leq 2$ within the $1 \sigma$ uncertainties.

We also employed a quantitative measure of the chance that the candidate $\lambda 2803$ transition could be a random feature or a transition from another system 8 along the QSO sight line. A "false alarm" probability is computed by scanning the spectrum pixel by pixel (also with an aperture given by the full width at the continuum of the candidate $\lambda 2796$ feature). The scan is performed about $50 \AA$ to both sides of the candidate $\lambda 2803$ feature. This corresponds to a to-

\footnotetext{
${ }^{8}$ We have performed an exhaustive cross-checking of line identifications from CIV and MgII redshifts reported in the literature and from those discovered in our spectra. Details of the line identifications and the list of searched transitions and redshifts will be presented in CVC98.
}

tal redshift window of $\Delta z \sim 0.02$, or $\pm 3000 \mathrm{~km} \mathrm{~s}^{-1}$ about the feature. The false alarm probability is simply the fraction of pixels with detected features (both emission and absorption) having a significance level greater than or equal to the candidate $\lambda 2803$ feature.

\subsection{Defining the Doublet Sample}

The criteria by which a candidate MgII doublet is determined is described above. In practice, the detection levels of the $\lambda 2803$ transitions for all included doublets were greater then $4.5 \sigma$. Often, other species, particularly strong FeII transitions and the MgI $\lambda 2853$ transition, were detected. We did not consider the detection of FeII or MgI as part of the selection criteria for our adopted sample.

Most bonafide Mg II doublets have false alarm probabilities of $P_{\mathrm{fa}} \leq 10^{-6}$. We found nine candidate MgII doublets with $10^{-6} \leq P_{\mathrm{fa}} \leq 0.05$. Most of these had unphysical doublet ratios or their wavelengths did not align within $3 \sigma$ of their uncertainties; thus they were removed from the candidate list. The largest false alarm probability for a Mg II doublet in our adopted sample is $P_{\mathrm{fa}} \sim 0.009$ (see discussion of $\mathrm{S} 24$ in Q1213 - 003 at $z=1.1277$ ). Visual inspection of this system reveals it to be robust; the relatively 


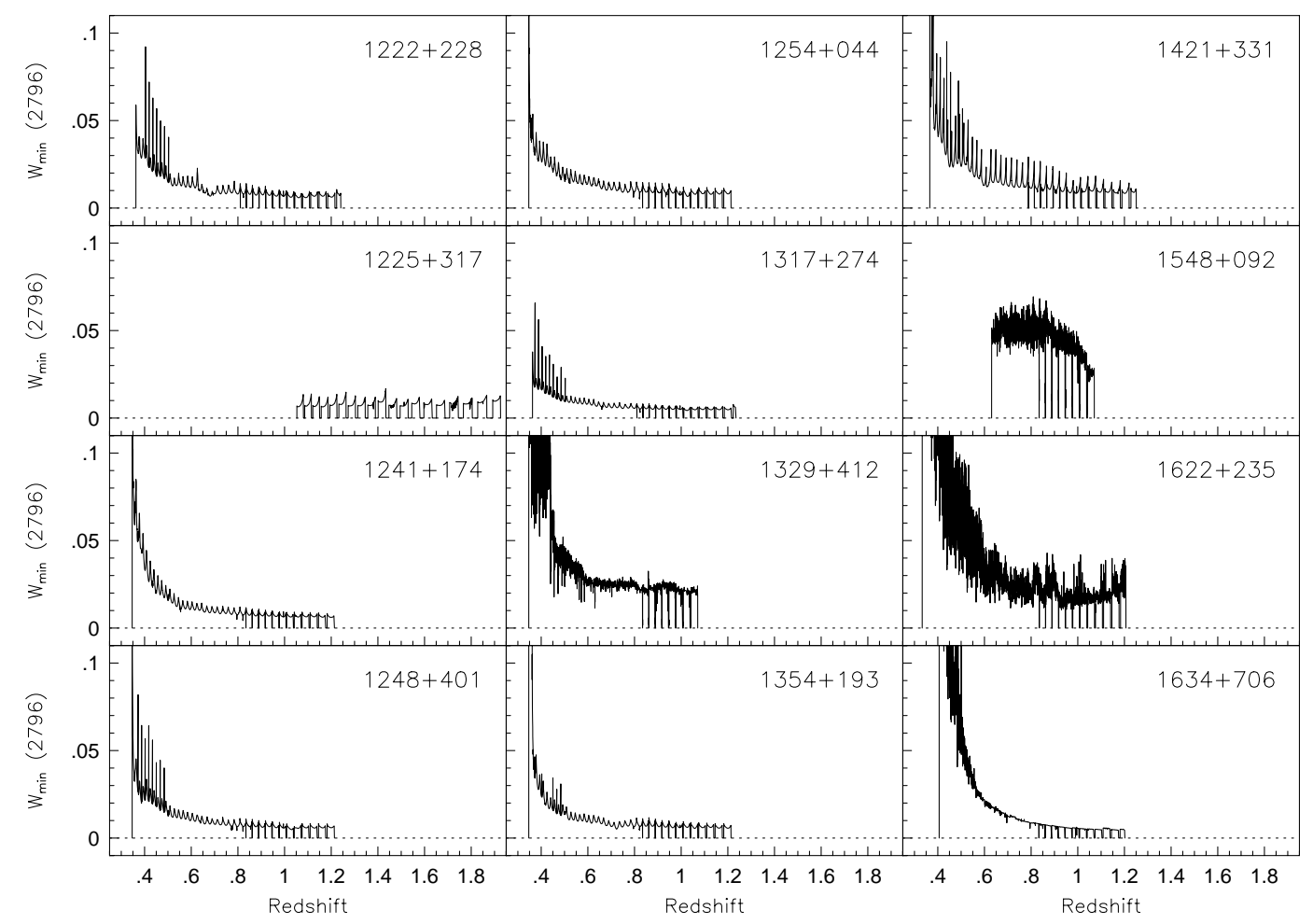

Fig. 1b. - Same as for figure 10

large $P_{\mathrm{fa}}$ is due to the number of features of equal or greater significance within the $6000 \mathrm{~km} \mathrm{~s}^{-1}$ window. Taken at face value, this implies that the S24 absorber had a $0.9 \%$ chance of being a false detection. We are quite certain that our adopted sample is not contaminated by false Mg II systems.

In addition to the above constraints, a nearest neighbor velocity separation of greater than $500 \mathrm{~km} \mathrm{~s}^{-1}$ was enforced. Of the strong MgII absorbing systems studied by CVC98, two had a maximum velocity spread slightly greater than $450 \mathrm{~km} \mathrm{~s}^{-1}$, if they are in fact single systems. The velocity filter was applied so that small equivalent width, high velocity components in strong systems would not be included in the sample. In practice, the $500 \mathrm{~km} \mathrm{~s}^{-1}$ separation criterion resulted in our dropping only a single potential weak MgII absorber from our sample (see discussion of S19 in the Q1206 + 459 spectrum). Had we applied a minimum velocity separation of $800 \mathrm{~km} \mathrm{~s}^{-1}$, we would have obtained the same sample.

\section{The Doublet Sample}

The adopted sample is presented in Table2. Tabulated are the system number, the absorption redshift, the QSO spectrum in which it was detected, the velocity width in $\mathrm{km} \mathrm{s}^{-1}$ of the $\lambda 2796$ transition, the rest-frame equivalent width of the $\lambda 2796$ transition, and the $\mathrm{Mg}$ II doublet ratio. The last column contains the cumulative redshift path, $Z\left(W_{\mathrm{r}}, \mathrm{DR}\right)$, which is the total redshift path over which the tabulated system could have been detected in this survey (see Eq. 2). The velocity widths, $\omega_{v}$, are measured directly from the flux values according to

$$
\omega_{v}^{2}=\int_{v_{-}}^{v_{+}} \tau_{a}(v)(\Delta v)^{2} d v / \int_{v_{-}}^{v_{+}} \tau_{a}(v) d v,
$$

where $\tau_{a}(v)=\ln \left[I_{\mathrm{c}}(v) / I(v)\right]$ is the apparent optical depth (Savage \& Sembach 1991), $I_{\mathrm{c}}(v)$ is the continuum flux at velocity $v, I(v)$ is the measured flux at $v$, $\Delta v=v-\langle v\rangle$, and $\langle v\rangle$ is the velocity centroid of the absorption profile. The $\omega_{v}$ are mathematically equivalent to the Gaussian width of a normal distribution.

In Table 3, we present the properties of each system, including the transition identity, the observed wavelength, and the rest-frame equivalent width or its $3 \sigma$ upper limit. The equivalent widths and their uncertainties are computed using the methods of Sembach \& Savage (1992). Because the FeII and MgI $\lambda 2853$ transitions are the strongest and most commonly found in MgII absorbers, we have presented their limits for the individual systems. Other transitions are included when they have been detected at the $3 \sigma$ level or above. 


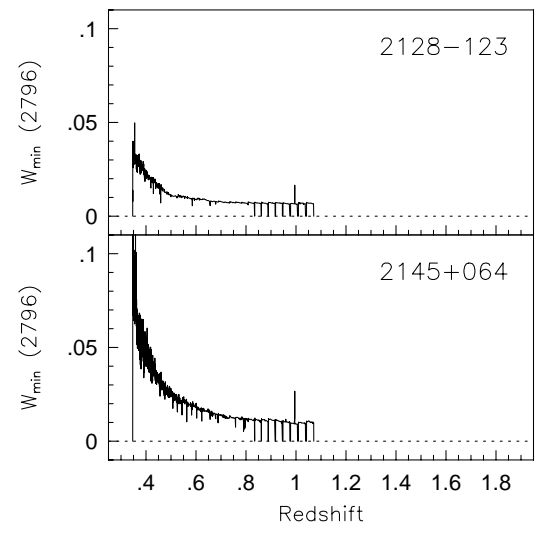

Fig. 1c.- Same as for figure 10

\subsection{Detection of Other Species}

\subsubsection{In the HIRES Spectra}

When a candidate Mg II doublet was identified, we searched for the presence of other transitions in absorption. Our search included the transitions presented in Table 1 (described in \$4.4). Since the search was based upon a priori knowledge of the MgII absorption redshift, the detection significance level was relaxed to $3 \sigma$. As seen in Figure $10-c$, the sensitivity is a strong function of redshift in the sense that we are less sensitive to lower redshift absorbers. As such, transitions with wavelengths blueward of the Mg II doublet were less likely to be detected, especially in the lower redshift systems.

\subsubsection{Literature Search and FOS/HST Archival Spec- tra}

We have searched for the CIV $\lambda \lambda 1548,1550$ doublet 9 at the redshifts of our Mg II sample. The purpose of the search was to obtain indications of high ionization in metal-line systems selected by weak Mg II absorption. From the literature, we found CIV was reported in three of the seven higher redshift systems in which it could be observed from the ground. The remaining four of the seven have not been observed.

With the kind assistance of A. Dobrzycki, we also

\footnotetext{
${ }^{9}$ We limited our search to C I. A _ more detailed search for other species will be presented in Paper II.
}

conducted a semi-rigorous search for the CIV doublet in the FOS/HST spectra of Dobrzycki et al. (1998). The $3 \sigma$ detection threshold of these spectra ranged from $W_{\mathrm{r}}^{\min }(1548)=0.15 \AA$ to $0.3 \AA$. We also searched the literature for reported CIV in FOS/HST spectra. We found CIV in six of the fifteen systems for which FOS/HST spectra were available.

In total, CIV was found or reported in nine of the 18 weak MgII systems for which information on CIV was available. Thus, it is reasonable to suggest that roughly half of all weak $\mathrm{Mg}$ II selected absorbers would show CIV absorption to the equivalent width thresholds available in existing data. We defer a discussion of the implications until $\$ 5.2$.

\subsection{Discussion of Systems}

The spectra for each of the detected transitions in the adopted MgII sample are shown in Figure 20$d d$. Only those formally detected with a greater than $3 \sigma$ level are shown. Features marked with a "*" are either members of other systems or are unidentified. In a companion paper (Churchill et al. 1998, hereafter Paper II), we will examine the detailed absorption properties on a system by system basis.

\subsection{1. $\mathrm{S} 1\left(\mathrm{Q} 1421+331 ; z_{\mathrm{abs}}=0.45642\right)$}

There is no previous report of S1 in the literature. No FeII transitions were captured by the HIRES CCD, only the MgII and MgI $\lambda 2853$ transitions with the latter not being detected. The Mg II profiles reveal a single feature that exhibits some unresolved saturation. There is a slight suggestion of blue asymmetry in the $\lambda 2803$ transition that may be due to multiple narrow components. However, the signal-to-noise ratio is low enough that the profile shape is not without some ambiguity. A FOS/HST spectrum of this QSO was not available for our CIV search. Though this field has been studied in some detail, to date there is no galaxy candidate associated with this absorber (C. Steidel, private communication).

\subsection{2. $\mathrm{S} 2\left(\mathrm{Q} 1329+412 ; z_{\mathrm{abs}}=0.500786\right)$}

S2 was first reported as a "probable" MgII doublet by SSB. The signal-to-noise ratio of the HIRES spectrum is fairly low, and only the Mg II doublet was detected. The limiting equivalent widths of the two captured FeII transitions are not very stringent. The profiles are resolved into a broad multi-component structure, particularly in the higher quality $\lambda 2796$ 


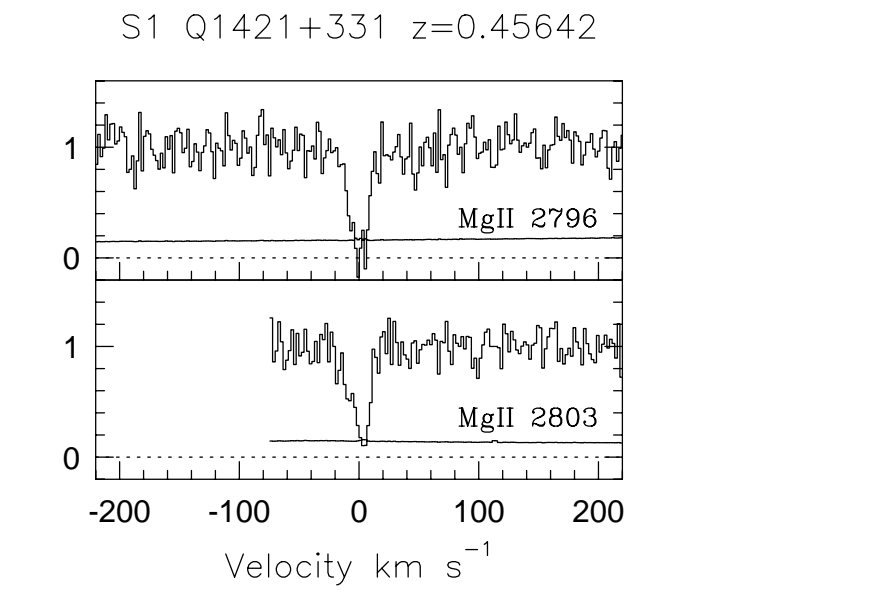

Fig. 2a.- The detected transitions of the weak Mg II systems. All detections are $5 \sigma$ unless annotated otherwise. Absorption features marked with "*" are not associated with the system being presented. In systems with mutliple components, ticks mark the components that are detected at the $3 \sigma$ level.

transition. Mg I was not detected. A FOS/HST spectrum of this QSO was not available for our C IV search. There is an associated galaxy with this absorber (C. Steidel, private communication).

$$
\text { S2 } Q 1329+412 z=0.50079
$$

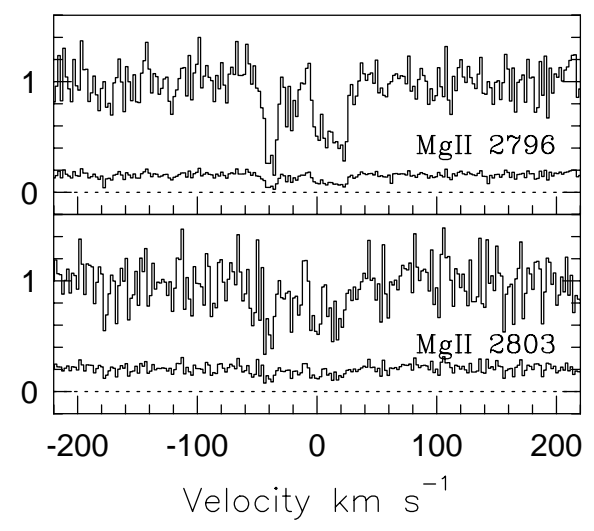

Fig. 2b.- Same as for figure 20

\subsection{3. $\mathrm{S} 3\left(\mathrm{Q} 1354+193 ; z_{\mathrm{abs}}=0.52149\right)$}

There is no previous report of $\mathrm{S} 3$ in the literature. The Mg II $\lambda 2796$ transition was coincident with a bad column in the HIRES CCD. This pixel has been re- placed by interpolating between its nearest neighbors and its uncertainty has been proportionately scaled to reflect the "correction factor". The MgII profile appears to be a single component that may be partially resolved in that there is a red--wing asymmetry in both transitions of the doublet. Though FeII and Mg I were captured by the CCD, only the MgII doublet was detected. CIV absorption was not detected in the FOS/HST spectrum (also see Jannuzi et al. 1998). Though this QSO field has about five galaxies all within $\pm 200 \mathrm{~km} \mathrm{~s}^{-1}$ of $z=0.45$, which are part of a foreground cluster, to date there is no galaxy candidate associated with this weak absorber (C. Steidel, private communication).

$$
\text { S3 Q1354+193 } z=0.52150
$$

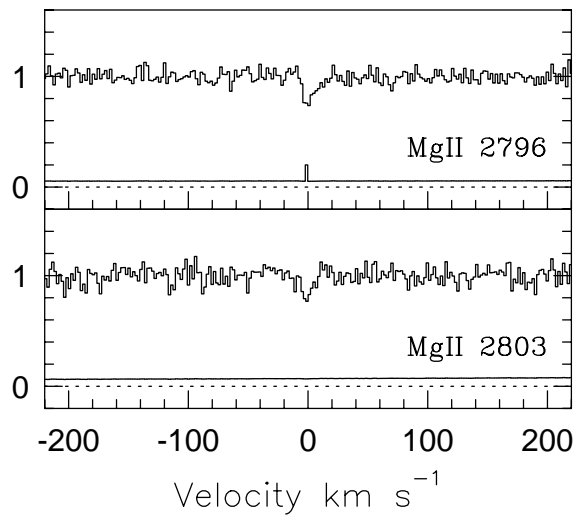

Fig. 2c.- Same as for figure 20

$$
\text { 3.2.4. } \mathrm{S} 4\left(\mathrm{Q} 1222+228 ; z_{\mathrm{abs}}=0.550202\right)
$$

There is no previous report of $\mathrm{S} 4$ in the literature. The MgII profile is well resolved with velocity dispersion of $\omega_{v} \sim 10 \mathrm{~km} \mathrm{~s}^{-1}$, and is likely comprised of multiple narrower components. It is fairly broad and shallow, such that the $\lambda 2803$ transition equivalent width could be sensitive to the continuum fit; it is possible the continuum fit could result in the doublet search algorithm missing or falsely detecting this system. Thus, the continuum fit was examined. Both a fit to the full echelle order and a more constrained fit to the region $\pm 500 \mathrm{~km} \mathrm{~s}^{-1}$ about the transition yielded $W_{\mathrm{r}}(2803)$ measurements consistent within $1 \sigma$ errors. For both fits, the $\lambda 2803$ transition had a greater than $5 \sigma$ detection and the doublet false alarm ratio was $\leq 10^{-6}$. Though FeII and MgI were captured by the CCD, only the MgII doublet was de- 
tected. However, the FeII $\lambda 2600$ transition may have been detected at the $2.7 \sigma$ level. CIV absorption was not detected in the FOS/HST spectrum (also see Impey et al. 1996). Though this field has been studied in some detail, to date there is no galaxy candidate associated with this absorber (C. Steidel, private communication).

$$
\text { S4 } Q 1222+228 z=0.55020
$$

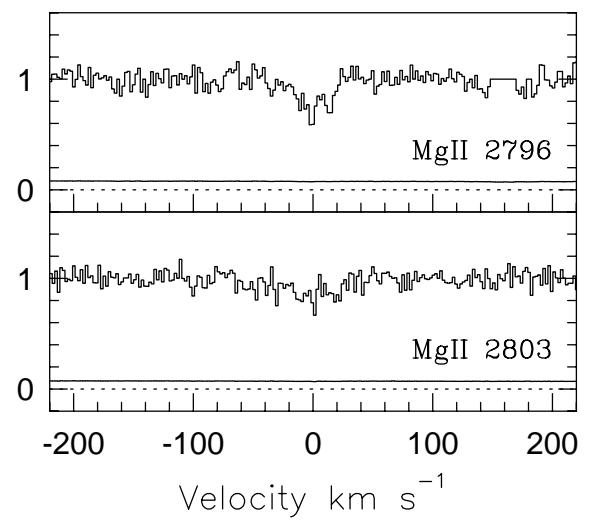

Fig. 2d.- Same as for figure 20

\subsection{5. $\mathrm{S} 5\left(\mathrm{Q} 1241+174 ; z_{\mathrm{abs}}=0.55844\right)$}

There is no previous report of $\mathrm{S} 5$ in the literature. The MgII profile is also well resolved with velocity dispersion of $\omega_{v} \sim 17 \mathrm{~km} \mathrm{~s}^{-1}$. Though FeII and Mg I were captured by the CCD, only the Mg II doublet was detected. Associated CIV absorption was tentatively detected in the FOS/HST spectrum from our search. However, the $\lambda 1548$ line may be Ly $\alpha$ Jannuzi et al. 1998). Though this field has been studied in some detail, to date there is no galaxy candidate associated with this absorber (C. Steidel, private communication).

\subsection{6. $\mathrm{S} 6\left(\mathrm{Q} 0002+051 ; z_{\mathrm{abs}}=0.59149\right)$}

S6 was discovered by C. Steidel and collaborators C. Steidel, private communication and is known to be associated with a red $\simeq 1.3 L_{K}^{*}$ galaxy with impact parameter $\simeq 24 h^{-1} \mathrm{kpc}$ Churchill, Steidel, \& Vogt 1996). The MgII profiles are partially resolved, at best, and are probably unresolved. Though multiple FeII transitions were captured by the CCD, none were detected. If $\mathrm{MgI} \lambda 2853$ is present, it was detected only at the $2 \sigma$ significance level. As mentioned above, the equivalent widths measured in this spectrum may
S5 Q1241+174 $z=0.55844$

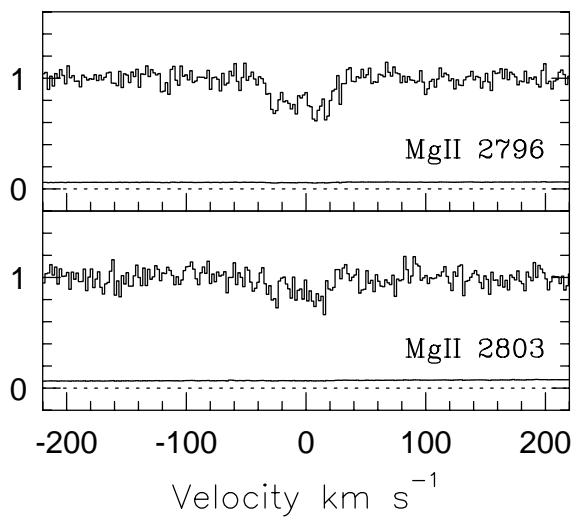

Fig. 2e.- Same as for figure 20

be biased by a zero-point uncertainty, which has not been included in the error measurement. CIV absorption was not detected in the FOS/HST spectrum (also see Jannuzi et al. 1998).

S6 Q0002+051 z=0.59149

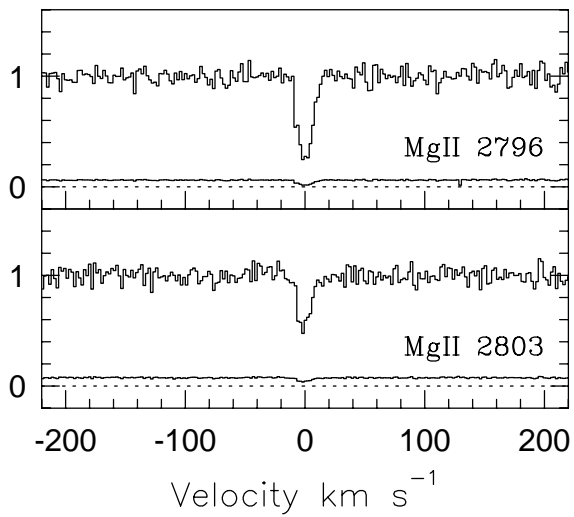

Fig. 2f.- Same as for figure 2

\subsection{7. $\mathrm{S} 7\left(\mathrm{Q} 0454+039 ; z_{\mathrm{abs}}=0.64283\right)$}

S7 was first reported by Churchill \& Le Brun (1998), who found it to have near-solar metallicity based upon photoionization modeling of MgII, FeII and the Ly $\alpha$ transition detected in the FOS/HST spectrum of Boissé et al. (1997). CIV absorption was not detected in the FOS/HST spectrum. Interestingly, there is no galaxy at this redshift in this well studied field (Churchill \& Le Brun 1998). The Mg II 
profiles, both symmetric with little structure, are apparently unresolved; there is slight unresolved saturation in the $\lambda 2796$ profile. Here, we also report a $3.3 \sigma$ detection of FeII $\lambda 2587 . \mathrm{Mg}$ I was not detected.

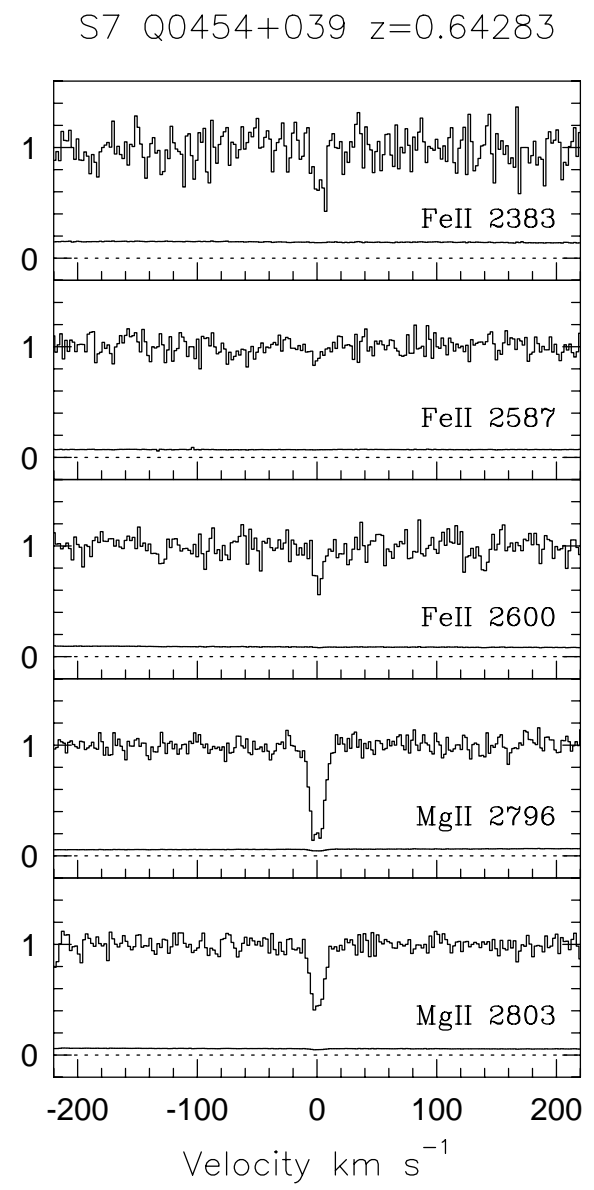

Fig. 2g.- Same as for figure 20

\subsection{8. $\mathrm{S} 8\left(\mathrm{Q} 0823-223 ; z_{\mathrm{abs}}=0.705472\right)$}

There is no previous report of S8 in the literature. The Mg II profiles reveal a single resolved feature with a small blueward asymmetry. Though FeII and Mg I were captured by the CCD, only the Mg II doublet was detected. A FOS/HST spectrum of this QSO was not available for our Civ search.

\subsection{9. $\mathrm{S} 9\left(\mathrm{Q} 0058+019 ; z_{\mathrm{abs}}=0.72518\right)$}

There is no previous report of S9 in the literature. The Mg II profiles are resolved, with $\omega_{v} \sim 11 \mathrm{~km} \mathrm{~s}^{-1}$, and saturated in their cores. This system is interesting in that relatively strong $\mathrm{Mg}_{\mathrm{I}} \lambda 2853$ was detected.
S8 Q0823-223 z=0.70547

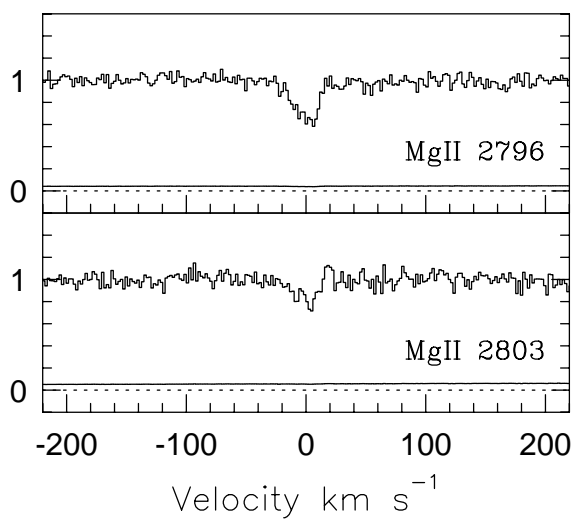

Fig. 2h.- Same as for figure 20

S9 $Q 0058+019 z=0.72518$

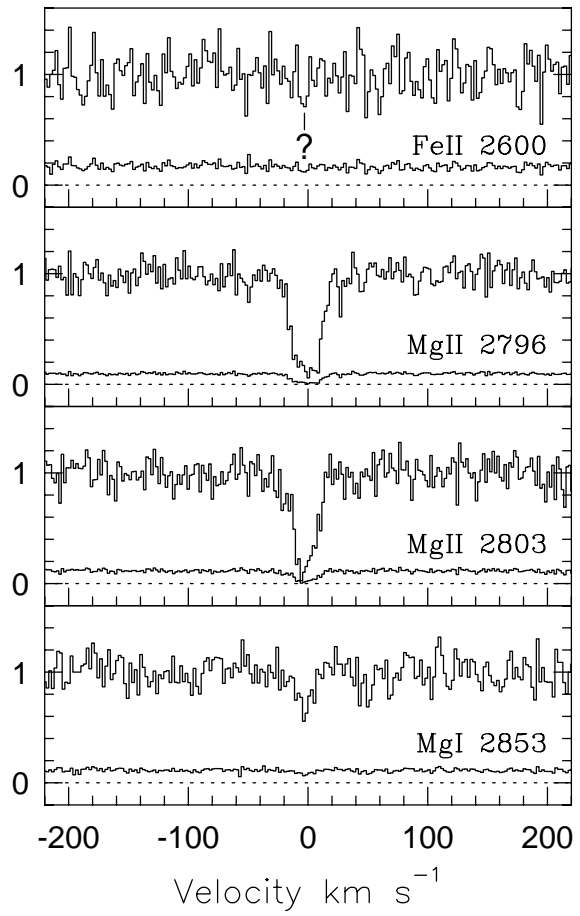

Fig. 2i.- Same as for figure 20

There is a tentative, $3 \sigma$ detection of FeII $\lambda 2600$, which we present in Table 3, but this detection is deemed insecure based upon the fact that there are three $3 \sigma$ detections in a velocity window of $\pm 500 \mathrm{~km} \mathrm{~s}^{-1}$ centered on the putative FeII detection. Moreover, there is no evidence to $3 \sigma$ for the stronger $\lambda 2383$ tran- 
sition, which lies in a region of the spectrum with a slightly lower signal-to-noise ratio. A FOS/HST spectrum of this QSO was not available for our CIV search. Though this field has been studied in some detail, to date there is no galaxy candidate associated with this absorber, though there is a galaxy (nonabsorbing) at $z \simeq 0.68$ (C. Steidel, private communication).

\subsubsection{0. $\mathrm{S} 10\left(\mathrm{Q} 0117+212 ; z_{\mathrm{abs}}=0.72907\right)$}

S10 was discovered by C. Steidel and collaborators (C. Steidel, private communication). It is associated with a quite red and massive $\sim 3.7 L_{K}^{*}$ galaxy at an impact parameter of $\sim 36 h^{-1} \mathrm{kpc}$ (Churchill et al. 1996). The absorption profiles are seen to be comprised of five unresolved weak components. The absorption at $v \simeq-120 \mathrm{~km} \mathrm{~s}^{-1}$ near the $\lambda 2803$ transition is a TiII transition from a damped $\operatorname{Ly} \alpha$

S10 Q0117+212 z=0.72907

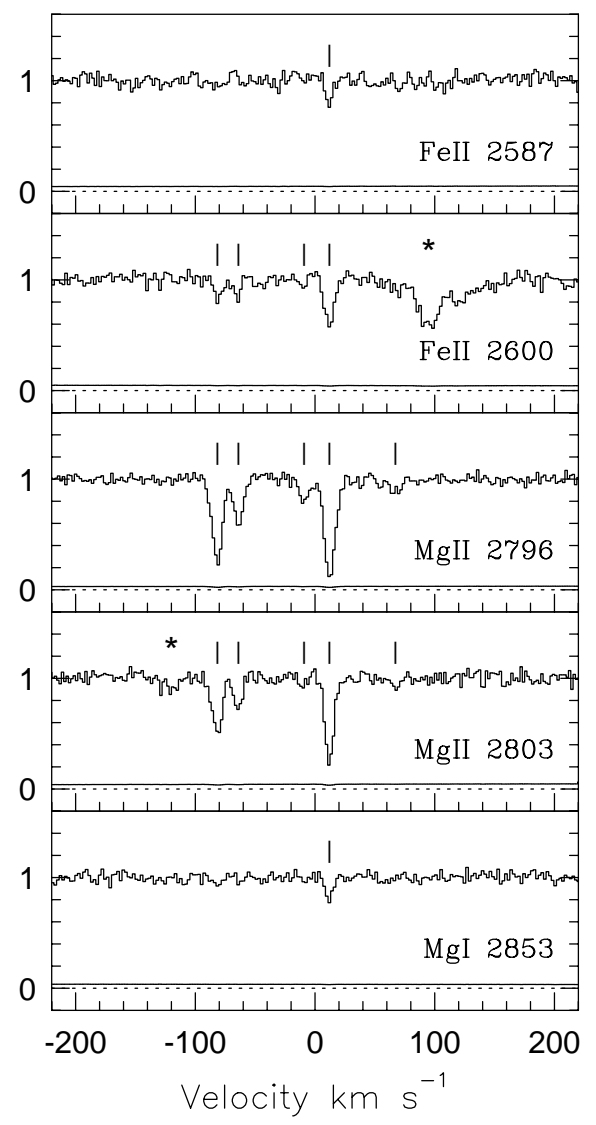

Fig. $2 j$.- Same as for figure 20 absorber at $z_{\text {abs }}=0.5764$ (CVC98). Also, the FeII $\lambda 2600$ is nearly blended with $\mathrm{Mg}$ I $\lambda 2853$ from this damped Ly $\alpha$ absorber. The overall system has a velocity dispersion of $\omega_{v} \sim 50 \mathrm{~km} \mathrm{~s}^{-1}$. FeII has been detected in four of the five MgII components and the MgI $\lambda 2853$ transition was detected in the strongest one. This system is interesting in that ratio of the FeII and MgI strengths may vary from component to component. CIV absorption was not detected in the FOS/HST spectrum (also see Jannuzi et al. 1998).

\subsubsection{1. $\mathrm{S} 11\left(\mathrm{Q} 1548+093 ; z_{\mathrm{abs}}=0.77065\right)$}

SSB reported a weak, but unambiguous, MgII absorbing system at $z_{\mathrm{abs}}=0.7708$. The HIRES spectrum is noisy and the detected absorption profiles suffer from unresolved saturation. However, the profiles are significantly broader than the HIRES instrumental profile and it is likely that multiple nar-

\section{S11 Q1548+092 $z=0.77065$}

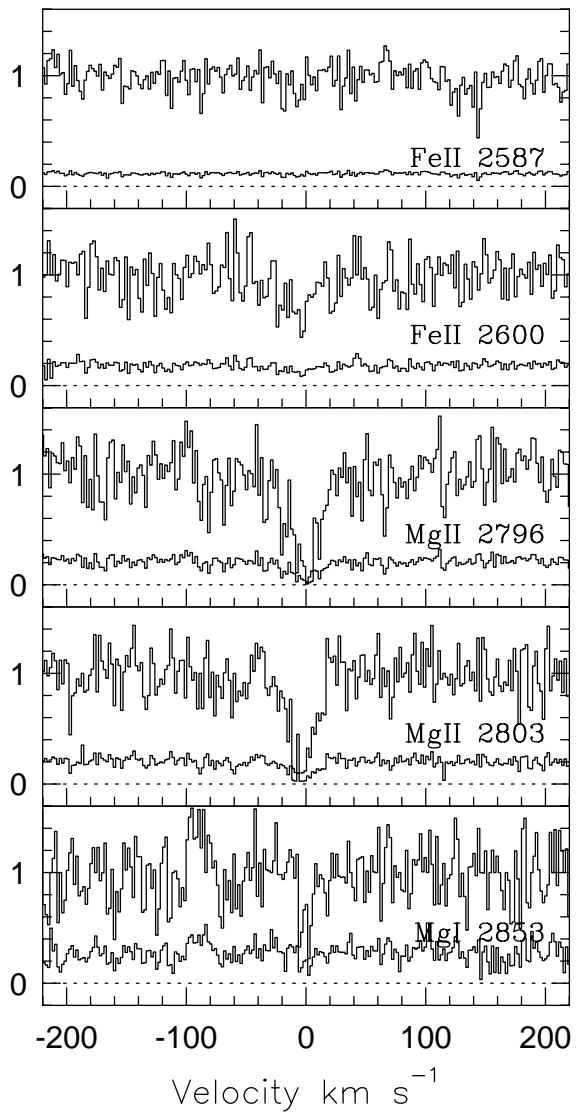

Fig. $2 k$ - - Same as for figure 20 
row components are present. There is a detection of Mg I $\lambda 2853$, even though this region of the spectrum is noisy. Some of the FeII transitions lie blueward of the Ly $\alpha$ emission line, and thus their detection is confused by the presence of many forest lines. However, the FeII $\lambda 2600$ and the weaker $\lambda 2587$ transitions are just redward of the Ly $\alpha$ emission line. In fact, this region of the spectrum has higher signal-to-noise ratio due to the elevated flux that results from the blend of the Ly $\alpha$ and Nv $\lambda \lambda 1238,1242$ emission lines. There is a detection of the $\lambda 2600$ transition and a $3.5 \sigma$ detection of the $\lambda 2587$ transition. A FOS/HST spectrum of this QSO was not available for our CIV search. There is an associated galaxy with this absorber (C. Steidel, private communication).

\subsubsection{2. $\mathrm{S} 12\left(\mathrm{Q} 1634+706 ; z_{\mathrm{abs}}=0.81816\right)$}

There is no previous report of S12 in the literature. The Mg II profiles are unresolved and very weak with $W_{\mathrm{r}}(2796)=0.03 \AA$. There is no detection of MgI, where the signal-to-noise ratio is quite high, nor of FeII. CIV absorption was not detected in the FOS/HST spectrum (also see Bahcall et al. 1996).

S12 Q1634+706 z=0.81816

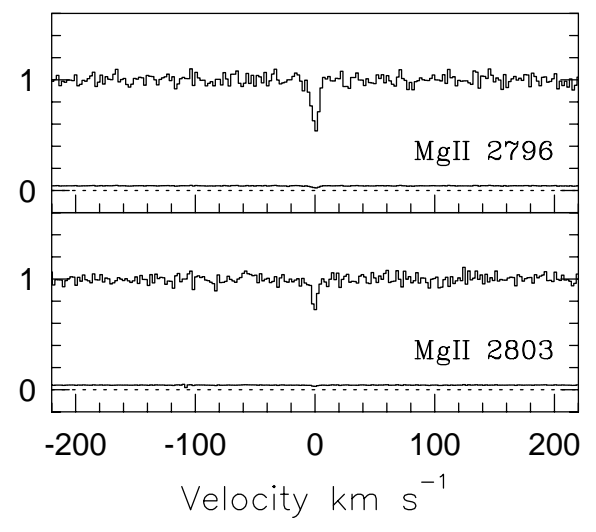

Fig. 2l. - Same as for figure 20

\subsubsection{3. $\mathrm{S} 13\left(\mathrm{Q} 1421+331 ; z_{\mathrm{abs}}=0.84325\right)$}

There is no previous report of S13 in the literature. However, Uomoto (1984) reported two unidentified weak lines in his low resolution spectrum; these two lines correspond to FeII $\lambda 2600$ and $\lambda 2374$ at this redshift. The MgII profiles are unresolved and saturated, which is also suggested by the unity doublet ratio.
S13 Q1421+331 $z=0.84325$

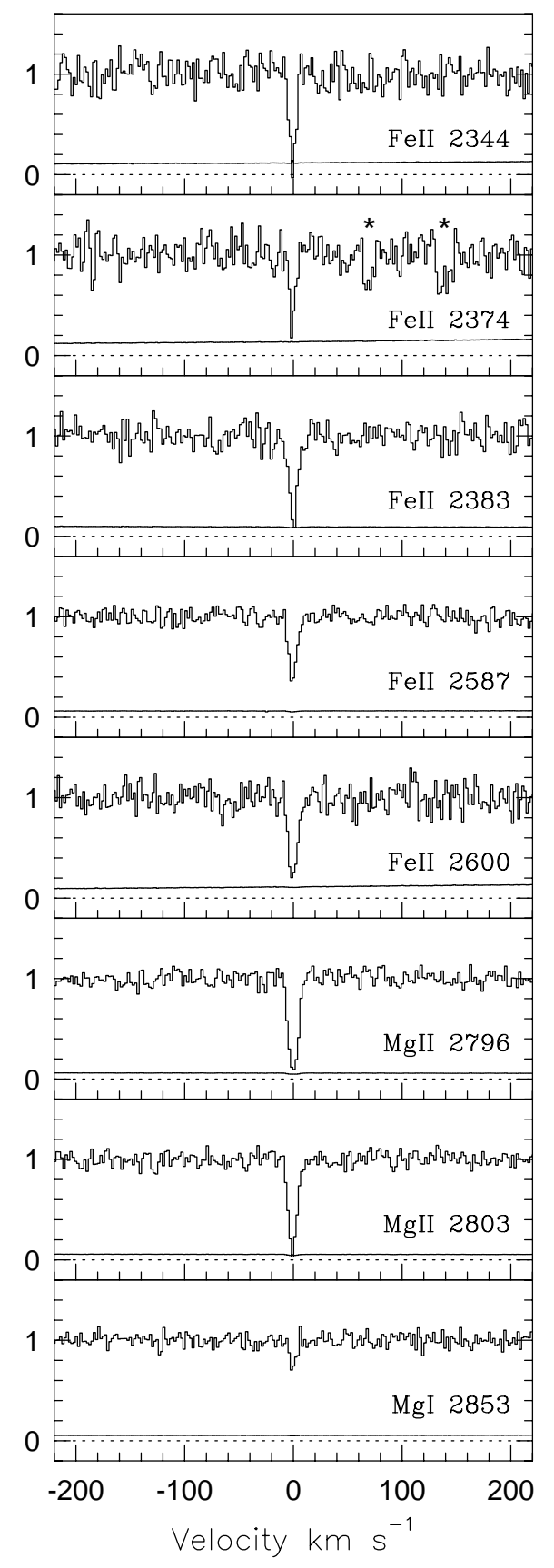

Fig. 2m. - Same as for figure 20 
This system is very rich in FeII transitions: $\lambda 2600$, 2587, 2383, 2374, and 2344 were all detected to $5 \sigma$. In fact, the FeII transitions are also unresolved and saturated. Also, MgI $\lambda 2853$ is detected. A FOS/HST spectrum of this QSO was not available for our C IV search. Given the very large strength of the FeII absorption, this system may be iron-group enhanced.

\section{$\mathrm{S} 14 \mathrm{Q} 1248+401 \quad \mathrm{z}=0.85455$}

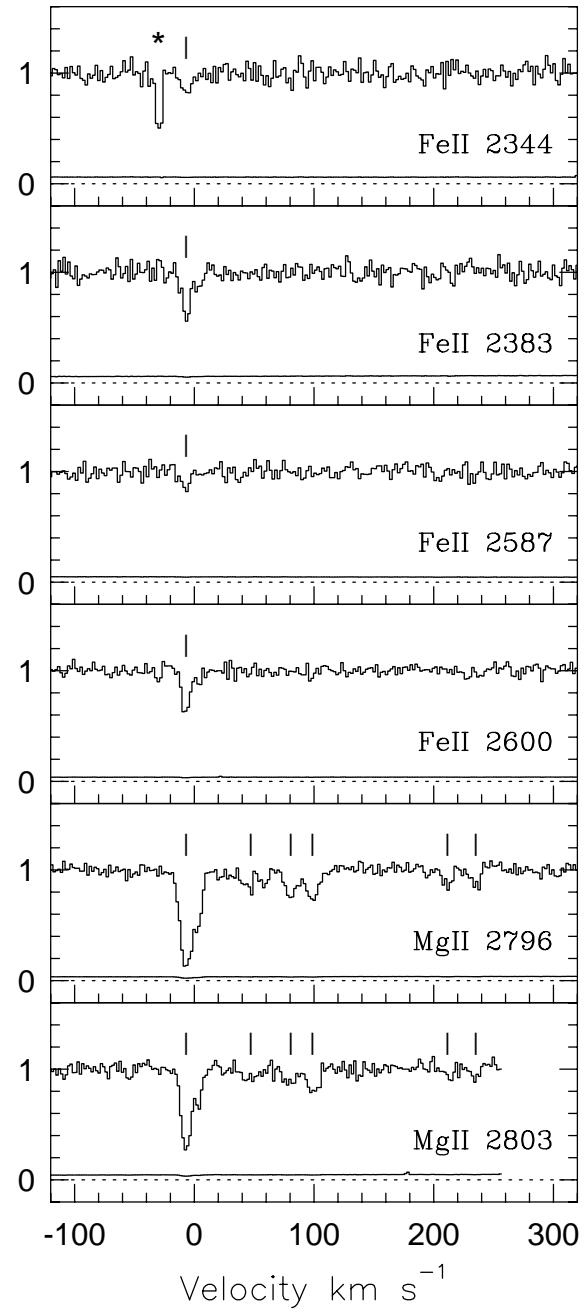

Fig. 2n.- Same as for figure $2 a$

\subsubsection{4. $\mathrm{S} 14\left(\mathrm{Q} 1248+401 ; z_{\mathrm{abs}}=0.85455\right)$}

There is no previous report of S14 in the literature. The MgII profiles are characterized by a central asymmetric absorption feature (clearly multiple component) and several weaker "high velocity" features.
There is also broad shallow absorption that stretches approximately $+100 \mathrm{~km} \mathrm{~s}^{-1}$ from this strong absorption profile. Altogether, there are three absorbing "subsystems", the others being unresolved and at high velocities $\left(v>+200 \mathrm{~km} \mathrm{~s}^{-1}\right)$. FeII was detected only in the strongest Mg II profile at $v \sim 0 \mathrm{~km} \mathrm{~s}^{-1}$ and also shows the redward double component asymmetry. If $\mathrm{Mg}$ I $\lambda 2853$ is present in absorption, we have detected it only at the $1.5 \sigma$ level. CIV absorption at this redshift was reported by Jannuzi et al. (1998) in the FOS/HST spectrum and is confirmed in our search. Though this field has been studied in some detail, to date there is no galaxy candidate associated with this absorber (C. Steidel, private communication).

\subsubsection{5. $\mathrm{S} 15\left(\mathrm{Q} 0002+051 ; z_{\mathrm{abs}}=0.86653\right)$}

There is no previous report of $\mathrm{S} 15$ in the literature. This is the weakest system found in our survey, with $W_{\mathrm{r}}(2796)=0.018 \AA$. The Mg II profiles are unresolved. We show the detection in Figure 3 in order to illustrate the robustness of our weakest detection in the sample. Though FeII and MgI were captured by the CCD, only the MgII doublet was detected. As mentioned above, the equivalent widths may be biased by a zero-point uncertainty, which has not been included in the error measurement. CIV absorption was not detected in the FOS/HST spectrum (also see Jannuzi et al. 1998). The Q0002 + 051 field has been studied in detail and there is no galaxy (to roughly $\left.0.2 L_{K}^{*}\right)$ observed at this redshift within 20 arcsec of the QSO (C. Steidel, private communication).
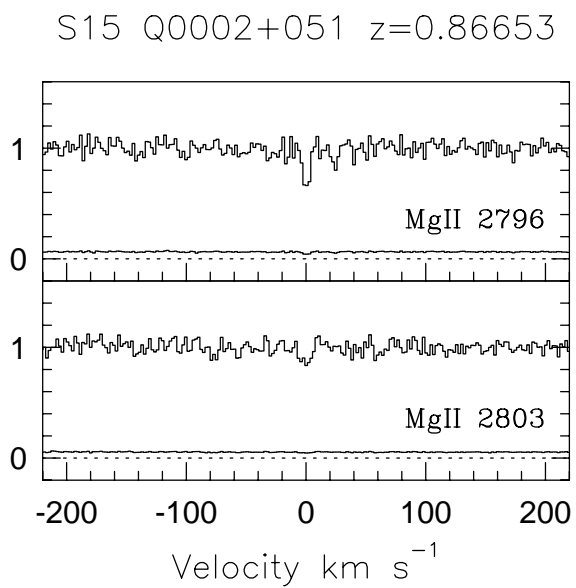

Fig. 2o.- Same as for figure 20 


$$
\text { 3.2.16. } \mathrm{S} 16\left(\mathrm{Q} 1241+174 ; z_{\mathrm{abs}}=0.89549\right)
$$

There is no previous report of S16 in the literature. The detection of S16 is quite similar to that of S15 (see Figure 3). However, in the case of S16 the profiles are broader, but still unresolved. The regions of the spectrum for five FeII transitions were captured by the CCD with high signal-to-noise ratios, but FeII was not detected. The Mg I $\lambda 2853$ transition was also not detected. CIV absorption was not detected in the FOS/HST spectrum (also see Jannuzi et al. 1998). Though this field has been studied in some detail, to date there is no galaxy candidate associated with this absorber (C. Steidel, private communication).
S17 Q1634+706 z=0.90555

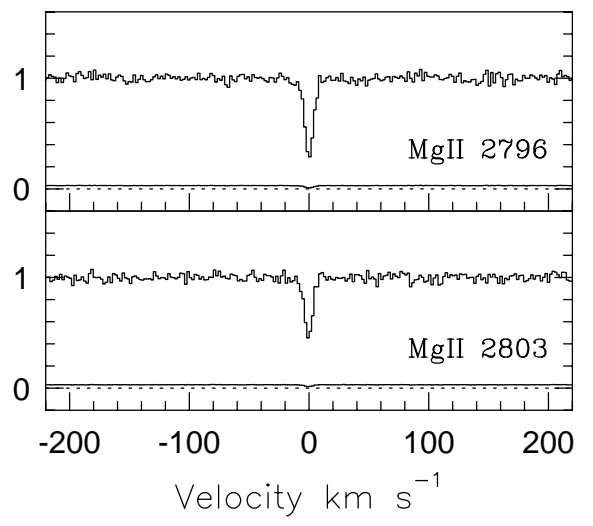

Fig. 2q.- Same as for figure 20

\& Le Brun 1998). The MgII profiles are unresolved. There appears to be a small defect in the blue wing of the $\lambda 2803$ transition, so that the measured $W_{\mathrm{r}}(2803)$ is $\sim 20 \%$ smaller than it would be if the pixel in question were not compromised or did not have a $\sim 3 \sigma$ noise spike (the profile presented in Figure 20 has not been smoothed or otherwise modified). The quoted doublet ratio and $W_{\mathrm{r}}(2803)$ in Table 3 are based upon the simulations described in Churchill \& Le Brun (1998). The FeII $\lambda 2383$ transition was clearly detected, and the FeII $\lambda 2600$ transition would likely

$$
\text { S18 Q0454+039 z=0.93150 }
$$

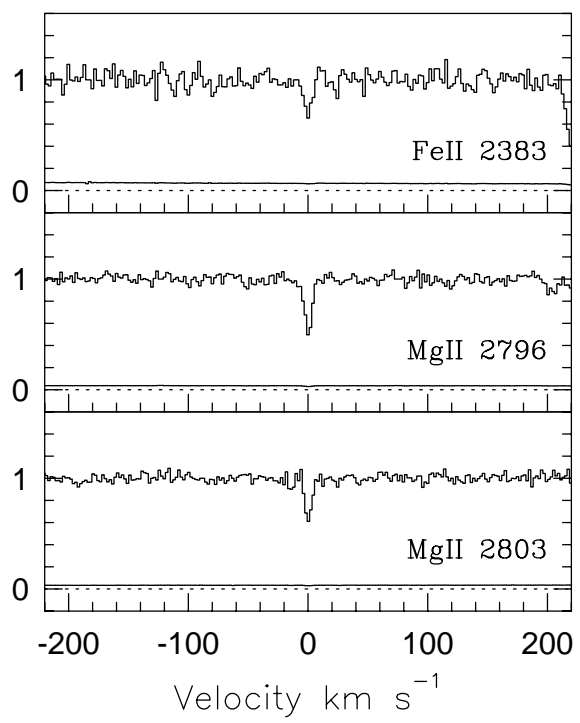

Fig. 2r.- Same as for figure 22 
have been detected, but the region of the spectrum was compromised by the infamous pen mark on the HIRES CCD. There is a tentative detection $(2.7 \sigma)$ of the weak FeII $\lambda 2587$ transition. The MgI $\lambda 2853$ transition was not detected.

\subsubsection{9. $\mathrm{S} 19\left(\mathrm{Q} 1206+459 ; z_{\mathrm{abs}}=0.93428\right)$}

There is no previous report of S19 in the literature. The MgII profiles may be partially resolved, given that their velocity width is $\omega_{v} \sim 8 \mathrm{~km} \mathrm{~s}^{-1}$. There is a blueward asymmetry to the profile that suggests there may be multiple velocity components. No FeII transitions were detected, nor was the MgI $\lambda 2853$ transition. The FeII $\lambda 2374$ transition is coincident with the FeII $\lambda 2383$ transition of a strong Mg II system at $z=0.9276$.

\section{S19 Q1206+459 $z=0.93428$}

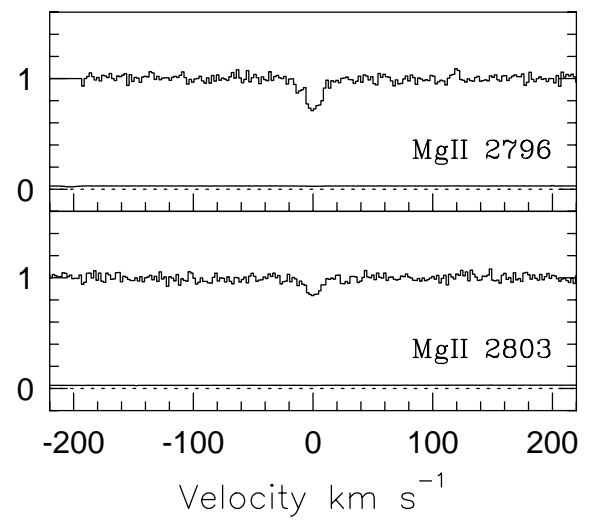

Fig. 2s.- Same as for figure 20

S19 may be a member of a small group of galaxies. Kirhakos et al. (1992) identified three galaxies within a few arc-seconds of the QSO. Thimm (1995) found high star formation rates on one of these galaxies based upon [OII] $\lambda 3727$ emission at $z=0.93$. There is a strong, kinematic-rich MgII system at $z_{\text {abs }}=0.9276$, a relative redshift of $\sim-2000 \mathrm{~km} \mathrm{~s}^{-1}$ with respect to S19. There is a third system, also with rich kinematics, about $\sim-450 \mathrm{~km} \mathrm{~s}^{-1}$ in redshift from the strong system. The measured $W_{\mathrm{r}}(2796)$ of this latter system is $0.22 \AA$; had the velocity separation of the latter two systems been $\sim 10 \%$ larger, the $0.22 \AA$ system would have been included into our survey. In the FOS/HST spectrum, high ionization transitions from CIV, Sirv, and Nv are prominent and spread over the full velocity range of the MgII absorbers (Churchill 1997b).

\subsubsection{0. $\mathrm{S} 20\left(\mathrm{Q} 0002+051 ; z_{\mathrm{abs}}=0.95603\right)$}

There is no previous report of S20 in the literature. The MgII profiles are partially resolved. There is a bit of structure in the $\lambda 2796$ profile suggestive of multiple, but very weak, components. On the other hand, the $\lambda 2803$ profile appears to have a red wing. Its shape is not inconsistent with a symmetric profile that is compromised by Poisson noise, but it may also further corroborate the multiple component appearance of the $\lambda 2796$ transition. Neither the FeII $\lambda 2383$ nor the $\lambda 2600$ transitions were detected, nor was the $\mathrm{Mg}$ I $\lambda 2853$ transition. As mentioned above, the equivalent widths may be biased by a zero-point uncertainty, which has not been included in the error measurement. Associated CIV absorption was detected in the FOS/HST spectrum (also see Jannuzi et al. 1998). The Q0002 + 051 field has been studied in detail and there is no galaxy (to roughly $0.2 L_{K}^{*}$ ) observed at this redshift within 20 arcsec of the QSO (C. Steidel, private communication).
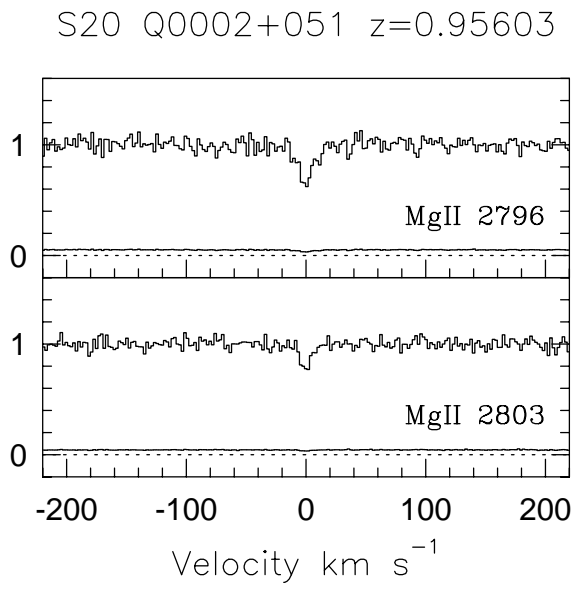

Fig. 2t.- Same as for figure 20

\subsubsection{1. $\mathrm{S} 21\left(\mathrm{Q} 1329+412 ; z_{\mathrm{abs}}=0.97387\right)$}

There is no previous report of S21 in the literature. The MgII profiles are resolved to have $\omega_{v} \sim$ $27 \mathrm{~km} \mathrm{~s}^{-1}$. They appear to be composed of several components. The $\lambda 2803$ transition is located very close to the CCD edge. Given the signal-to-noise ratio of the spectra in the region of the Mg II doublet, and because the profiles are broad and made up of 
weak components separated by continuum, this system could possibly have been missed by the doublet searching algorithm. For various continuum fits, the transition had a greater than $5 \sigma$ detection and the doublet false alarm ratio was $\leq 10^{-6}$. Neither FeII $\lambda 2383$ nor $\lambda 2600$ was detected. The $\operatorname{Mg}$ I $\lambda 2853$ transition may have been detected (at the $2.5 \sigma$ level). A FOS/HST spectrum of this QSO was not available for our CIV search. Though this field has been studied in some detail, to date there is no galaxy candidate associated with this absorber (C. Steidel, private communication).

\section{S21 Q1329+412 z=0.97387}

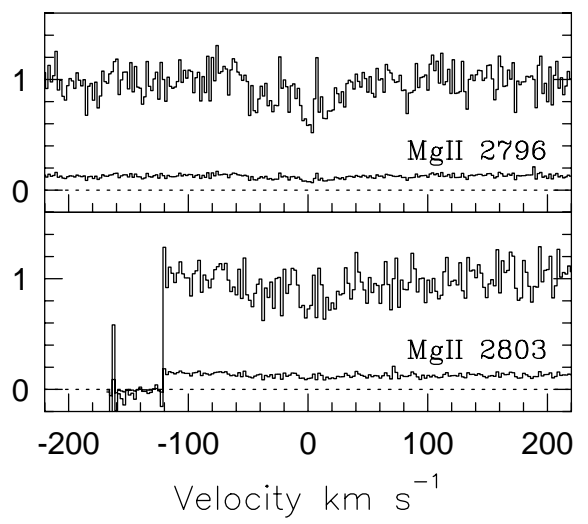

Fig. $2 u$. - Same as for figure 20

$$
\text { 3.2.22. } \mathrm{S} 22\left(\mathrm{Q} 1329+412 ; z_{\mathrm{abs}}=0.99836\right)
$$

There is no previous report of S22 in the literature. The MgII profiles are partially resolved and partially saturated. The profile shapes are fairly symmetric and there is no definitive evidence for multiple narrower components, but the profiles are suggestive of substructure. The system is strong in FeII absorption; the $\lambda 2600,2383$, and 2344 transitions were all detected to the $5 \sigma$ level (the $\lambda 2587$ transition was detected at $2.5 \sigma)$. The $\mathrm{Mg}$ I $\lambda 2853$ region of the spectrum was not captured by the CCD. A FOS/HST spectrum of this QSO was not available for our CIV search. Though this field has been studied in some detail, to date there is no galaxy candidate associated with this absorber (C. Steidel, private communication).
S22 Q1329+412 z=0.99836

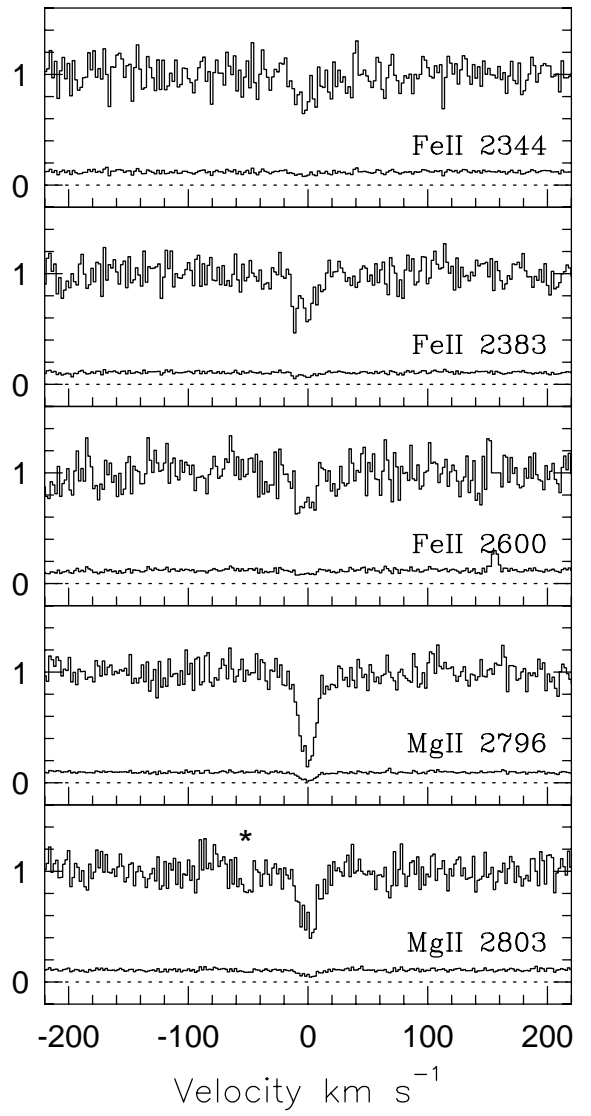

Fig. 2v.- Same as for figure 20

\subsubsection{3. $\mathrm{S} 23\left(\mathrm{Q} 1634+706 ; z_{\mathrm{abs}}=1.04144\right)$}

There is no previous report of detected Mg II in S23 in the literature. However, this system was reported by Bergeron et al. (1994) and Bahcall et al. (1996) to have Civ absorption. Even at very high signal-tonoise ratio, the FeII $\lambda 2600$, and 2383 transitions, and the $\operatorname{Mg}$ I $\lambda 2853$ transition were not detected.

\subsubsection{4. $\mathrm{S} 24\left(\mathrm{Q} 1213-003 ; z_{\mathrm{abs}}=1.12770\right)$}

There is no previous report of S24 in the literature. The MgII profiles are very narrow and unresolved. This system had a "false alarm" probability of $P_{\mathrm{fa}}=0.009$, which is the largest among our adopted sample. This is likely due to noise in the blue wing of the $\lambda 2803$ transition. The FeII $\lambda 2383$ transition may have been detected at the $3 \sigma$ level, but there are two features near the putative FeII line of comparable significance; they are also detected at the $3 \sigma$ level. We 
S23 Q1634+706 z=1.04144

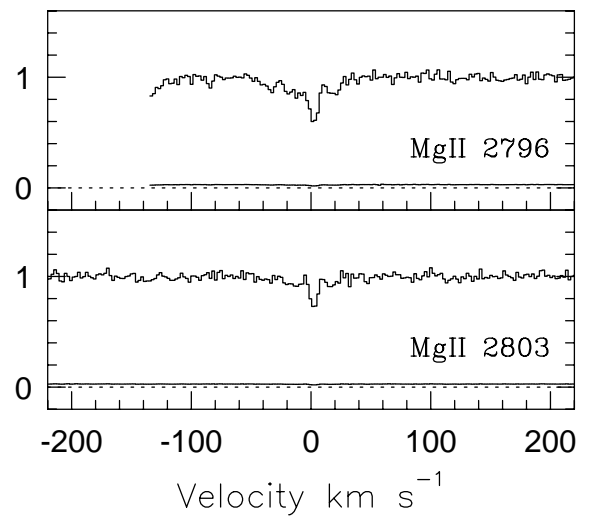

Fig. $2 w$ - - Same as for figure 20

$$
\text { S24 Q1213-003 z=1.12770 }
$$

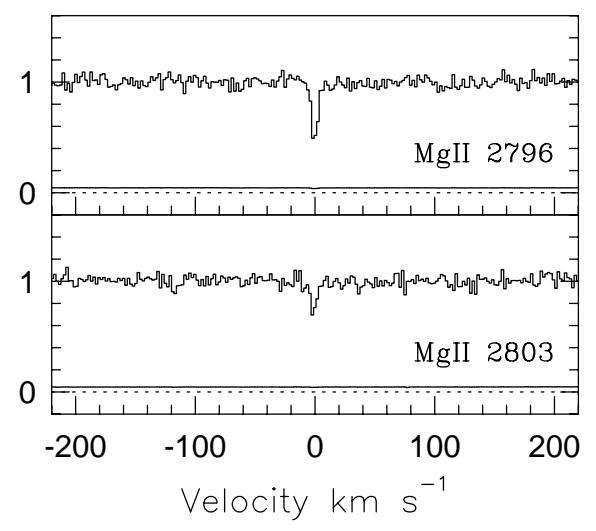

Fig. $2 x$. - Same as for figure $2 a$

conservatively quote a limit. A spectrum with the appropriate wavelength coverage for C IV at this redshift was not available for our CIV search.

\subsubsection{5. $\mathrm{S} 25\left(\mathrm{Q} 0958+551 ; z_{\mathrm{abs}}=1.21132\right)$}

There is no previous report of S25 in the literature. The MgII profiles are unresolved and symmetric. There is no detection of the FeII $\lambda 2600$ and $\lambda 2587$ transitions, nor of the $\mathrm{Mg}$ I $\lambda 2853$ transition. A spectrum with the appropriate wavelength coverage for CIV at this redshift was not available for our CIV search.
S25 Q0958+551 z=1.21132

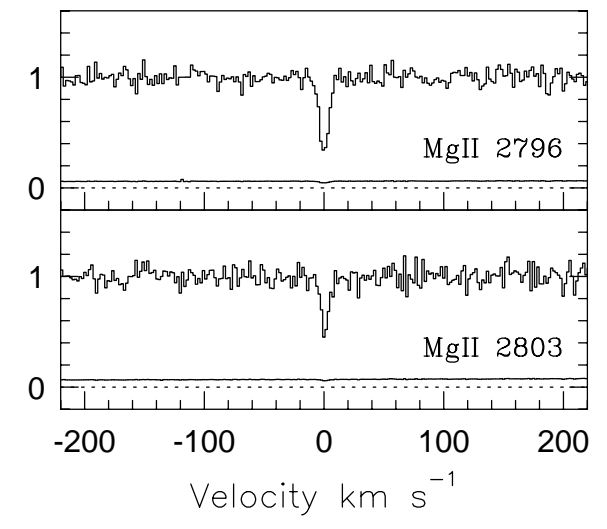

Fig. 2y.- Same as for figure 20

$$
\text { 3.2.26. } \mathrm{S} 26\left(\mathrm{Q} 0450-132 ; z_{\mathrm{abs}}=1.22948\right)
$$

S26 was first reported by SS92. The MgII profiles are resolved into two components, a main saturated component and a weaker component at $\sim$ $-35 \mathrm{~km} \mathrm{~s}^{-1}$. The system is strong in FeII absorption: the $\lambda 2600,2383$, and 2344 transitions were all detected to the $5 \sigma$ level in the main Mg II component. However, the $\lambda 2587$ transition was not detected to the $2.5 \sigma$ level, nor was the Mg I $\lambda 2853$ transition. No FeII was detected in the weaker MgII component. The AlıI $\lambda \lambda 1855,1863$ doublet was detected in the strong component (also see Petitjean, Rauch, \& Carswell 1994). This is the lowest redshift system in which the AlIII transitions were covered. S26 had a relative co-moving velocity of $-900 \mathrm{~km} \mathrm{~s}^{-1}$ with respect to S27. This is the smallest redshift separation of any two absorbers in the sample. A spectrum with the appropriate wavelength coverage for C IV at this redshift was not available for our CIV search. 
S26 Q0450-132 z=1.22948

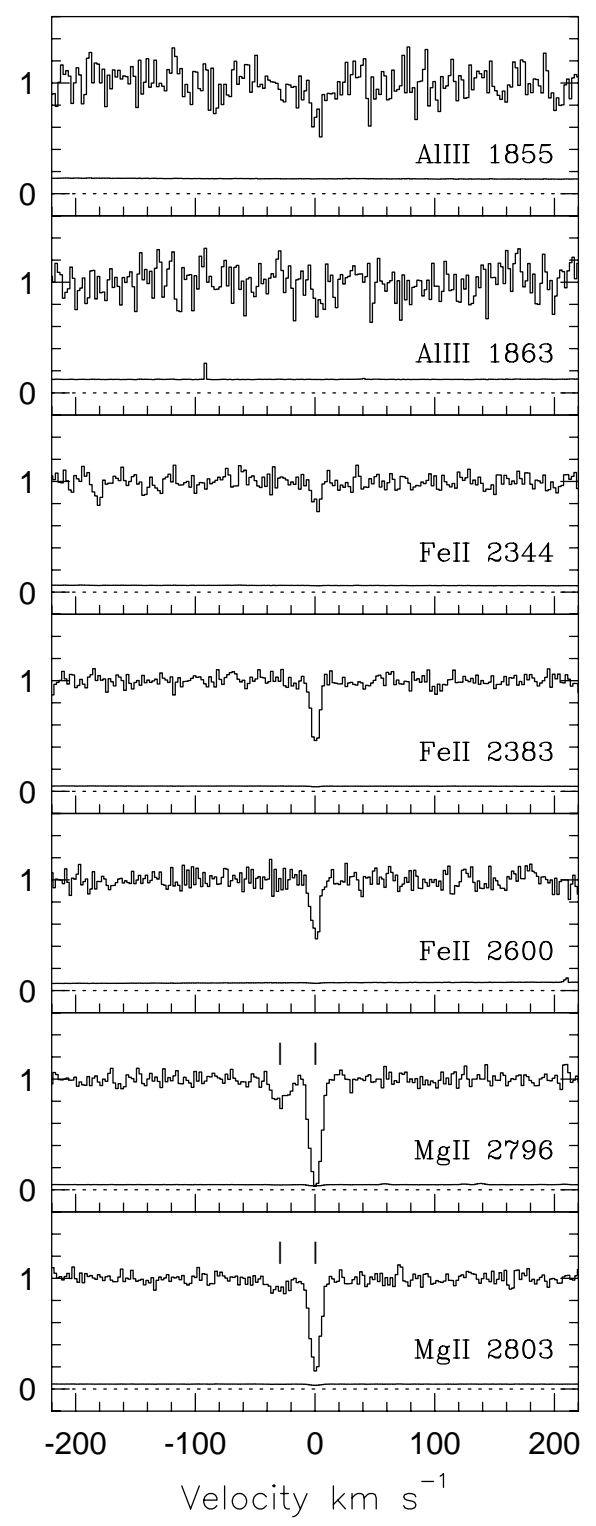

Fig. 2z.- Same as for figure 20

\subsubsection{7. $\mathrm{S} 27\left(\mathrm{Q} 0450-132 ; z_{\mathrm{abs}}=1.23244\right)$}

There is no previous report of S27 in the literature. The MgII profiles are also resolved into two components, a main unresolved narrow component and a shallow broad component at $\sim+65 \mathrm{~km} \mathrm{~s}^{-1}$. The FeII $\lambda 2383$, FeII $\lambda 2587$, and $\operatorname{Mg}$ I $\lambda 2853$ transitions were detected in the narrow component at the $5 \sigma, 4.3 \sigma$, and $3.5 \sigma$ level. There is some ambiguity as to the reality of the MgI detection. S26 had a relative co-moving velocity of $+900 \mathrm{~km} \mathrm{~s}^{-1}$ with respect to S27. A spectrum with the appropriate wavelength coverage for CIV at this redshift was not available for our Civ search.

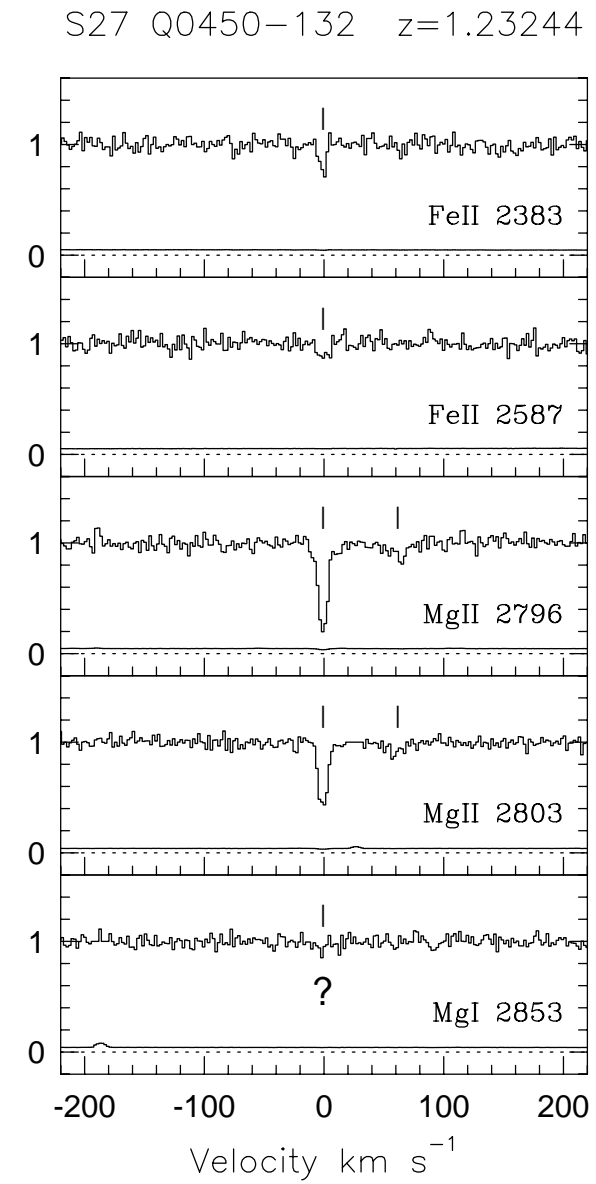

Fig. 2aa.- Same as for figure 2

\subsubsection{8. $\mathrm{S} 28\left(\mathrm{Q} 0958+551 ; z_{\mathrm{abs}}=1.27238\right)$}

There is no previous report of S28 in the literature. The MgII profiles are unresolved. The FeII $\lambda 2383$ transition has been detected. Also, the FeII $\lambda 2600$ transition has been detected at the $3.7 \sigma$ level. This $\lambda 2600$ transition falls in a relatively noisy region of the spectrum (off blaze), and thus its measured equivalent width is less certain than that of the apparently weak $\lambda 2383$ transition. In fact, the $\lambda 2600$ equivalent width is unphysically large with regard to the more robust $\lambda 2383$ equivalent width. There is no detection of the FeII $\lambda 2587$ and $\lambda 2374$ transitions. The $\operatorname{Mg}$ I $\lambda 2853$ 
transition may have been detected at the $3 \sigma$ level, but the detection is uncertain. Sargent, Boksenberg, \& Steidel (1988) reported CIV at this redshift.

\section{S28 Q0958+551 $z=1.27238$}

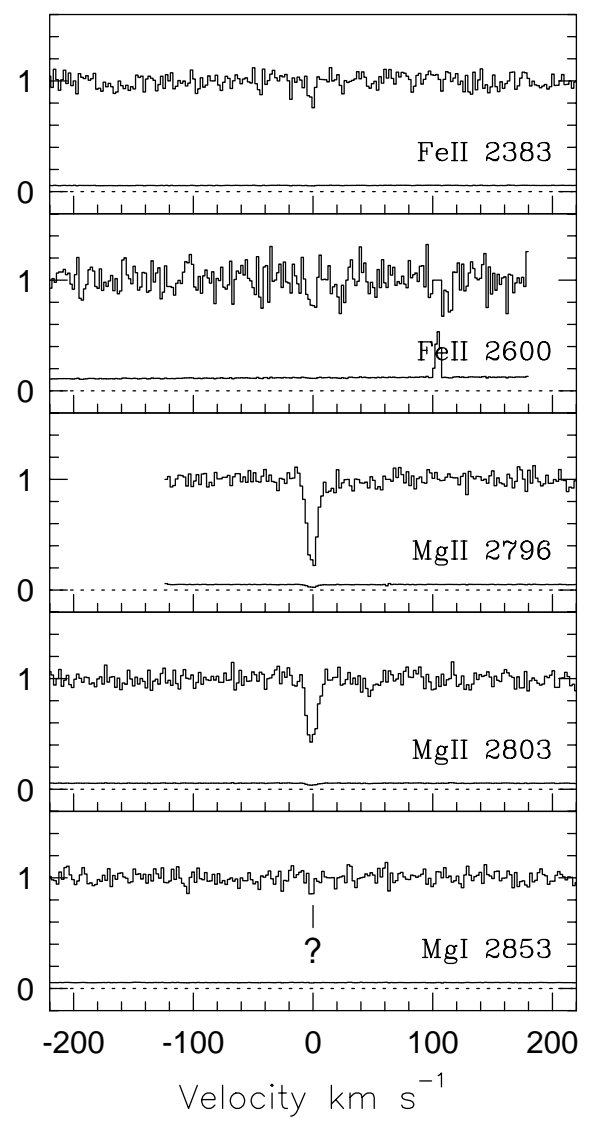

Fig. 2bb.- Same as for figure 20

\subsubsection{9. $\mathrm{S} 29\left(\mathrm{Q} 0117+212 ; z_{\mathrm{abs}}=1.32500\right)$}

S29 was first reported by SS92. The system is kinematically intriguing in that it is composed of five distinct components uniformly separated in velocity $\left(\Delta v \sim 30 \mathrm{~km} \mathrm{~s}^{-1}\right)$. The FeII $\lambda 2600,2587$, and 2383 transitions were detected in three of the five components. The FeII $\lambda 2374$ transition was not detected in any. The AlıII $\lambda 1863$ transition was discovered in three components; the AlIII $\lambda 1855$ transition was not captured by the CCD. There is evidence for variations in the chemical and/or ionization conditions in the five components; the strongest MgII component with FeII has the weakest FeII absorption. For the MgII components having comparable strengths, one has detectable FeII whereas the other does not. Mg I $\lambda 2853$ was detected at the $4.4 \sigma$ level, but only in the weakest Mg II component, and in this component,

$$
\text { S29 Q0117+212 z=1.32500 }
$$

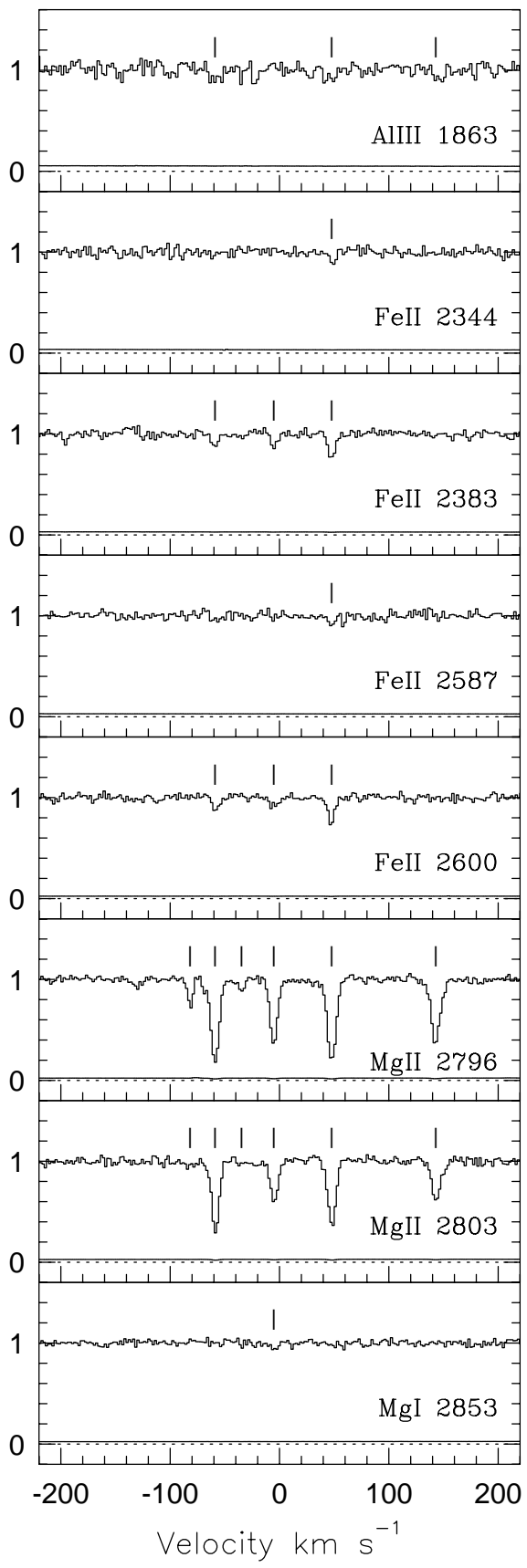

Fig. 2cc.- Same as for figure 20 
there is no Alini absorption. CIV absorption was reported by SS92, though the doublet was not resolved. The study of this QSO field is in progress. A few galaxies having properties consistent with their being at $z>1$ are seen, so that there are candidate galaxies for this system (C. Steidel, private communication).

\subsubsection{0. $\mathrm{S} 30\left(\mathrm{Q} 0117+212 ; z_{\mathrm{abs}}=1.34297\right)$}

S30 was also first reported by SS92. Only the $\lambda 2803$ transition of the $\mathrm{Mg}$ II doublet was captured. As such, this system would not have been detected in our unbiased doublet search. It is not a member of our adopted sample nor was it included in any of the system statistics. The MgII profile reveals multiple isolated components. Only the MgII $\lambda 2803$ transition was captured on the CCD. Thus, there is some missing information about the $\mathrm{Mg}$ II profile shapes given that the $\lambda 2796$ transition is the stronger of the doublet. There are four components, which are dominated by a narrow asymmetric profile. There is no detection of the FeII $\lambda 2374$ transition, nor of the $\mathrm{Mg}$ I $\lambda 2853$ transition. The FeII $\lambda 2587,2383$, and 2344 transitions have been detected in the dominant, but narrow, MgII component. Also, the AlıII $\lambda \lambda 1855,1863$ doublet was discovered in this component. CIV absorption was reported by SS92, though the doublet was not resolved. The study of this QSO field is in progress. A few galaxies having properties consistent with their being at $z>1$ are seen, so that there are candidate galaxies for this system (C. Steidel, private communication).

\subsection{Survey Completeness}

To evaluate the completeness of the survey as a function of redshift and to establish the threshold of detecting MgII doublets, we have adopted the formalism used by SS92 and LTW, namely the "redshift path density", $g(W, z)$. This function gives the number of sight lines along which a Mg II $\lambda 2796$ transition at redshift $z$ and with rest-frame equivalent width greater than or equal to $W$ could have been discovered.

As presented in the Appendix, we have slightly modified the computation and meaning of the redshift path density because of the high resolution of the spectra and the nature of the echelle data. The modifications include accounting for: (1) breaks in redshift coverage (which occur at absorption redshifts greater than $\sim 0.83$ ), (2) redundant redshift coverage (where
S30 Q0117+212 z=1.34297

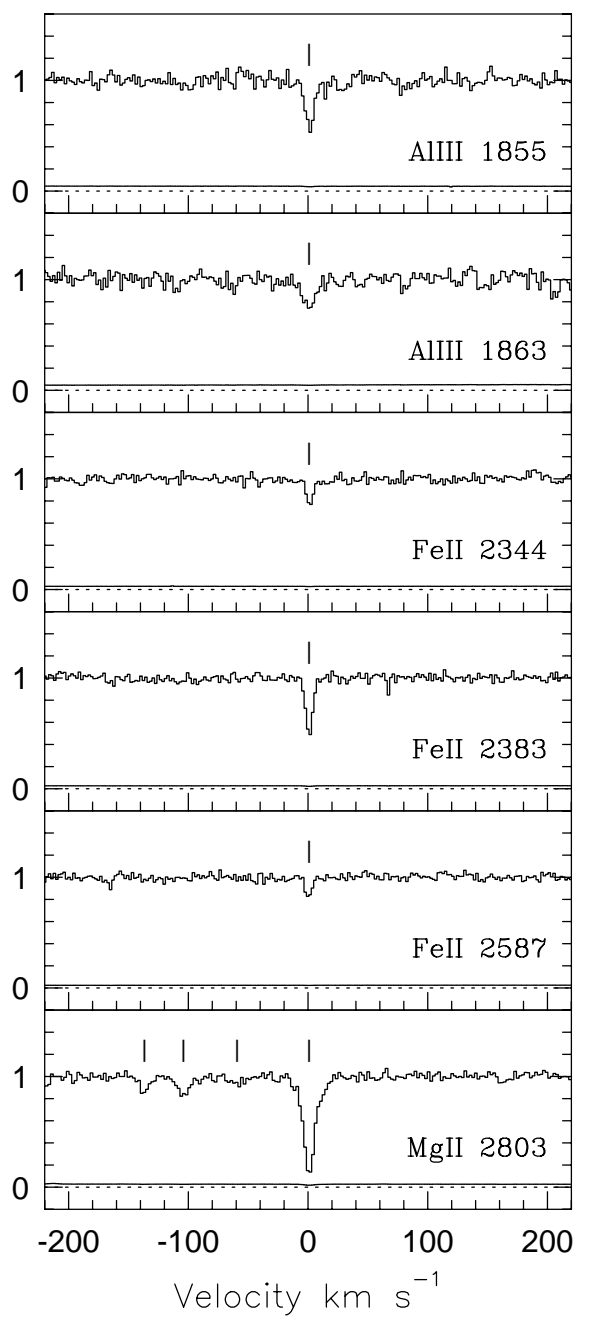

Fig. $2 d d$. - Same as for figure $2 d$

the CCD captured the same spectral regions on two adjacent echelle orders), and (3) the range of doublet ratios observed in the sample. Thus, we have defined the redshift path density, $g(W, z, \mathrm{DR})$, as the number of sight lines along which a MgII doublet at redshift $z$ with $\lambda 2796$ rest-frame equivalent width greater than or equal to $W$, and with doublet ratio less than DR could have been detected. The computation of $g(W, z, \mathrm{DR})$ is detailed in the Appendix.

The cumulative redshift path length covered by the survey over a given redshift interval is then

$$
Z\left(W^{\mathrm{min}}, \mathrm{DR}^{\max }\right)=\int_{z_{1}}^{z_{2}} g\left(W^{\mathrm{min}}, z, \mathrm{DR}^{\max }\right) d z
$$



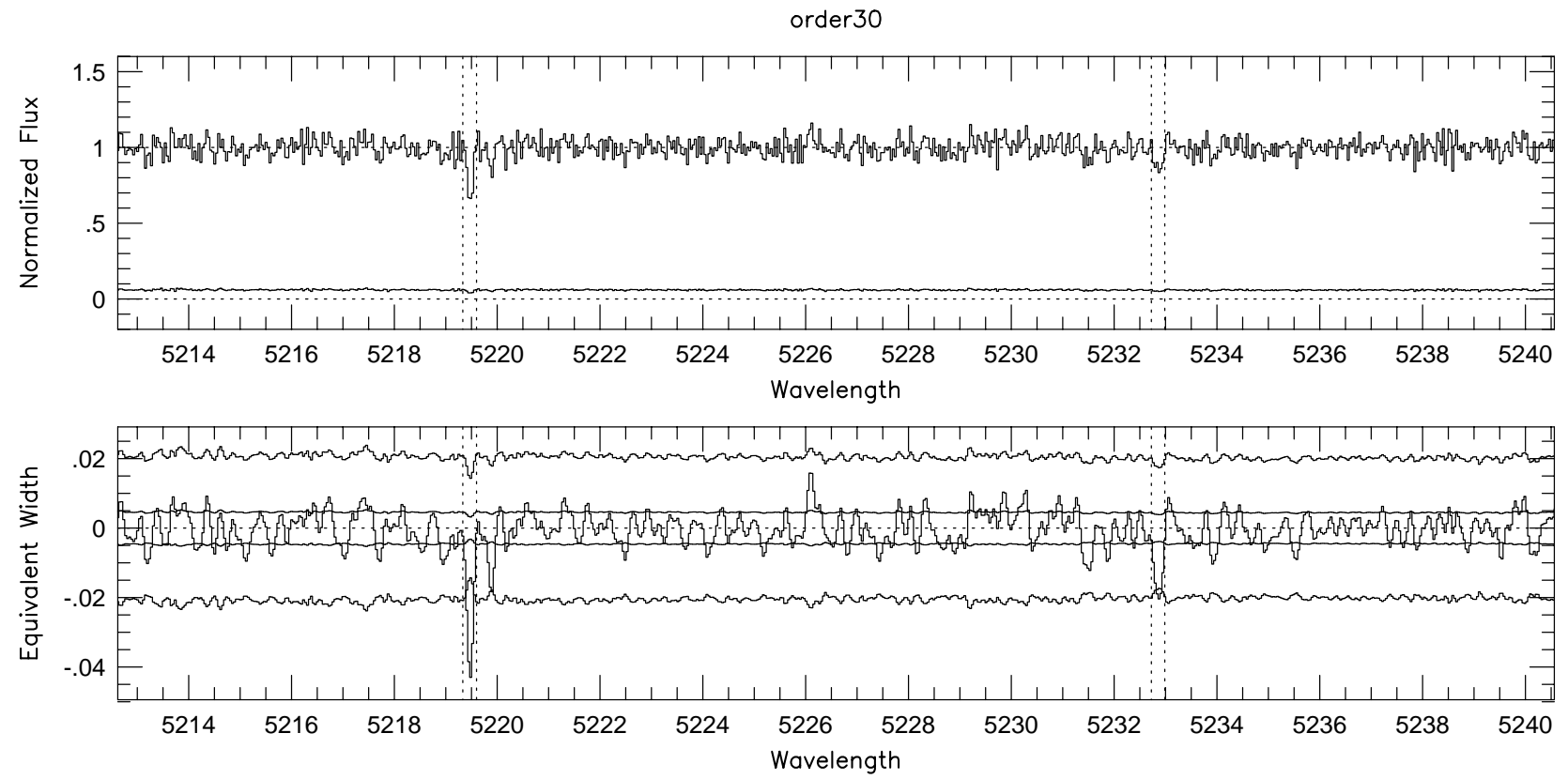

Fig. 3.- The S15 MgII doublet detection ( $5 \sigma)$ illustrated from our detection software. S15 is the weakest system in the sample and the (unresolved) profile shapes of the $\lambda 2796$ and $\lambda 2803$ transitions are quite different, with the $\lambda 2803$ profile being broader. This system provides an example of the detection sensitivity of our doublet searching algorithm. The limiting observed equivalent width on this order is roughly $0.02 \AA$, which corresponds to $0.011 \AA$ in the rest frame of the $\lambda 2796$ transition at these wavelengths. The top panel shows the spectrum and the uncertainty spectrum. The lower panel shows the equivalent width spectrum (average of zero). Pixels with positive equivalent widths are emission features and those with negative equivalent widths are absorption features. The uncertainty in the equivalent width spectrum is shown at both $1 \sigma$ (inner) and $5 \sigma$ (outer) levels. A feature is objectively identified when a pixel has an equivalent width that is larger than the $5 \sigma$ uncertainty. The vertical dashed lines are to illustrate the locations of the identified features.

where we have chosen not to integrate from $0 \leq z \leq$ $\infty$ because our $g\left(W^{\text {min }}, z, \mathrm{DR}^{\max }\right)$ drops dramatically below $z_{1} \leq 0.4$ and above $z_{2} \geq 1.4$. In fact, we did not detect any Mg II doublets outside this redshift range. In Figure 4, we have plotted the cumulative redshift path of the survey $(0.4 \leq z \leq 1.4)$ as a function of $W^{\min }$ for $\mathrm{DR}^{\max }=1$ (dotted curve) and for $\mathrm{DR}^{\max }=2$ (solid curve). It is apparent that the redshift path, and thus the survey completeness, is not sensitive to the doublet ratio. We are $91 \%$ complete at $W_{\mathrm{r}}(2796)=0.03 \AA$ (using a $5 \sigma$ detection criterion for the $\lambda 2796$ transition), and $80 \%$ complete at $W_{\mathrm{r}}(2796)=0.02 \AA$. For comparison, SS92 were $83 \%$ complete at $W_{\mathrm{r}}(2796)=0.3 \AA$ for a $5 \sigma$ detection level. Proper treatment of the redshift gaps was important. If the gaps had not been included in the computation of $Z$, then the cumulative redshift path leveled out at $\sim 18.3$ as opposed to $\sim 17.3$, though the shape as a function of $W^{\text {min }}$ was not altered from that presented in Figure 4 .

\section{The Statistical Properties of Weak Mg II Absorbers}

\subsection{Redshift Number Density}

Since we are unbiased only for $W_{\mathrm{r}}(2796) \leq 0.3 \AA$, we have adopted this limited range for our computation of $d N / d z$. Following LTW, we computed the number density by accounting for the fact that absorbers of different strengths could have been detected over different redshift path lengths. Thus, using Eq. 2, we computed the redshift path length of our survey over which each of the observed systems in our sample could have been detected. The values of 


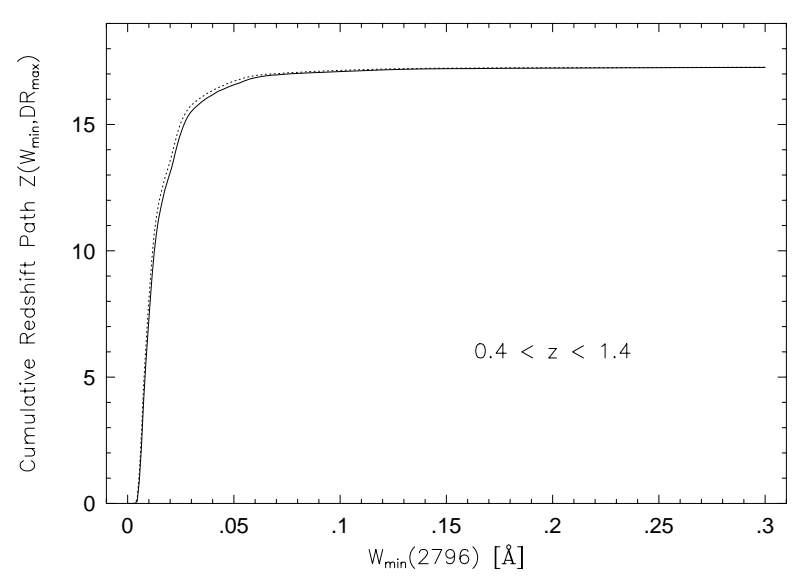

Fig. 4. - The redshift path of the survey over the redshift interval $0.4 \leq z \leq 1.4$ verses the rest-frame Mg II $\lambda 2796$ equivalent width threshold for a $5 \sigma$ detection level. The dotted curve is for unit doublet ratio and the solid curve is for $\mathrm{DR}=2$.

these $Z\left(W_{i}, D R_{i}\right)$ integrated over the redshift range $0.4 \leq z \leq 1.4$ are given in Table 2. The number per unit redshift path is simply the sum of the reciprocal of the cumulative redshift path lengths,

$$
\frac{d N}{d z}=\sum_{i}^{N_{\mathrm{sys}}}\left[Z\left(W_{i}, D R_{i}\right)\right]^{-1},
$$

where $W_{i}$ is the rest-frame equivalent width of the Mg II $\lambda 2796$ transition of the $i$ th system, and $\mathrm{DR}_{i}$ is its doublet ratio. The variance in $d N / d z$ is given by

$$
\sigma_{d N / d z}^{2}=\sum_{i}^{N_{\mathrm{sys}}}\left[Z\left(W_{i}, D R_{i}\right)\right]^{-2} .
$$

Over the redshift range $0.4 \leq z \leq 1.4$, we obtained $\frac{d N}{d z}=1.74 \pm 0.11 \quad$ for $0.02 \leq W_{\mathrm{r}}(2796)<0.3 \AA$,

where $\langle z\rangle=0.9$, and for which, as emphasized, the number applies to MgII systems with $0.02 \leq$ $W_{\mathrm{r}}(2796) \leq 0.3 \AA$. In the left hand panel of Figure 5 , we have plotted the redshift path density verses redshift for $0.02 \leq W_{\mathrm{r}}(2796) \leq 0.3 \AA$, where the computed $d N / d z$ points are in the three redshift bins $[0.40,0.74],[0.74,1.07]$, and $[1.07,1.40]$. The curves represent the no-evolution expectations for $q_{0}=0.5$ (dashed curve) and for $q_{0}=0$ (dotted curve) normalized to $d N / d z=1.74$ at $z=0.9$. We have assumed the standard parameterization,

$$
\frac{d N}{d z}=N_{0}(1+z)^{\gamma}
$$

where $\gamma=1$ for $q_{0}=0$ and $\gamma=0.5$ for $q_{0}=0.5$. A formal fit of the data to Eq. 6 yielded $\gamma=1.3 \pm 0.9$ and $N_{0}=0.8 \pm 0.4$, which is quite unconstraining. The data are not inconsistent with the no-evolution expectations for either $q_{0}$. In the right hand panel of Figure 5 , we have plotted the mean $d N / d z$ and its uncertainty at $\langle z\rangle=0.9$ for: (1) the $W_{\mathrm{r}}^{\min }(2796)=$ $0.3 \AA$ MG1 sample of SS92, which has $d N / d z=0.91 \pm$ 0.10 ; (2) the combined $W_{\mathrm{r}}^{\min }(2796)=0.02 \AA$ sample, which has $d N / d z=2.65 \pm 0.15$; and (3), the HST Key Project results for a sample of Lyman limit systems (LLS), which has $d N / d z=0.7 \pm 0.2$ over the interval $0.4 \leq z \leq 1.4$ (Stengler-Larrea et al. 1995).

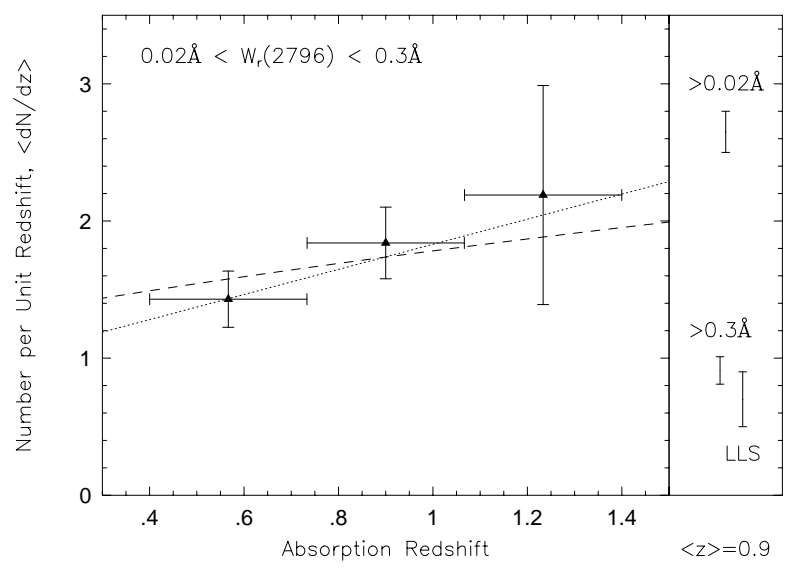

Fig. 5. - (left panel) The number of Mg II systems per unit redshift, $d N / d z$, with $0.02 \leq W_{\mathrm{r}}(2796) \leq 0.3 \AA$ for three redshift bins over the interval $0.4 \leq z \leq 1.4$. The vertical error bars are the Poisson uncertainties in the $d N / d z$ and the horizontal give the redshift bins. The dotted curve is the no-evolution expectations for $q_{0}=0$ and the dashed curve is for $q_{0}=0.5$, where the curves have been normalized at $z=0.9$ (see text). - (right panel) A comparison of the $\mathrm{Mg}$ II $d N / d z$ for different $\lambda 2796$ equivalent width cutoffs with the LLS $d N / d z$. The $W_{\mathrm{r}}(2796) \geq 0.3 \AA$ number is taken from SS92, and the Lyman limit data are taken from Stengler-Larrea et al. (1995).

Taken at face value, these numbers imply that weak MgII absorbers comprise $\sim 65 \%$ of the total MgII $a b$ sorber population and that the vast majority of them 
must arise in sub-LLS environments. We return to this point in $\$ 5.1$. The $d N / d z$ of weak $\mathrm{MgII}$ absorbers is roughly $5-7 \%$ of that of the $\operatorname{Ly} \alpha$ forest with $W_{\mathrm{r}}(\operatorname{Ly} \alpha) \geq 0.1 \AA$ (Jannuzi et al. 1998). We tentatively suggest that $\sim 5 \%$ of $z \leq 1$ "Ly $\alpha$ forest clouds" with $0.1 \leq W_{\mathrm{r}}(\operatorname{Ly} \alpha) \leq 1.6 \AA$ will exhibit $\mathrm{Mg}$ II absorption to a $5 \sigma W_{\mathrm{r}}(\lambda 2796)$ detection limit of $0.02 \AA$. The two MgII systems found by Churchill \& Le Brun (1998) in a search through 28 forest clouds in the spectrum of PKS $0454+039$ are consistent with these expectations.

Given the trend found by SS92 for the mean $d N / d z$ to increase steadily as the $W_{\mathrm{r}}^{\min }(2796)$ of their sample was decreased from $0.6 \AA$ to $0.3 \AA$ (see their Figure 5 ), it is not surprising that our survey has yielded the above results. Our large $d N / d z$ would be expected if the equivalent width distribution continued to increase as a power law for $W_{\mathrm{r}}(2796) \leq 0.3 \AA$. However, several investigators have previously examined the lower end of the MgII equivalent width distribution (SS92; BP90; Caulet 1989; SSB; LTW; TBSYK), and have claimed evidence of a lower cut off just below $0.3 \AA$. Extrapolating the steep power-law distribution reported by TBSYK, PB90 compared the number of expected absorbers with the number they actually detected, and reported a paucity of weak systems. By examining three narrow equivalent width ranges, $[0.45,0.60],[0.30,0.45]$, and $[0.15,0.30] \AA$, SS92 found $d N / d z$ dropped for the $[0.15,0.30] \AA$ bin (however, this is a $\sim 1 \sigma$ signal).

More recently there has been preliminary evidence that the distribution of equivalent widths continues to rise. Womble (1995) reported five Mg II systems with $W_{\mathrm{r}}(2796) \geq 0.015 \AA$ in a HIRES/Keck spectrum ${ }^{\text {TO }}$ of $\mathrm{Q} 1634+706$ over the interval $0.52 \leq z \leq 1.34$. For a sample with $W_{\mathrm{r}}^{\min }(2796)=0.03 \AA$, Tripp, et al. (1997), tentatively reported $d N / d z=2.3 \pm 0.8$. Their large uncertainty is due to the small cumulative redshift path covered by their spectra $(Z=2.56)$. We discuss the implications of a large mean number density per unit redshift of weak MgII systems in $\$$.

\subsection{Equivalent Width Distribution}

The distribution function, $n(W)$, is defined as the number of $\mathrm{MgII}$ absorption systems with equivalent width $W$ per unit equivalent width per unit redshift

\footnotetext{
${ }^{10}$ For comparison, we have found three systems with $W_{\mathrm{r}}(2796) \geq$ $0.03 \AA$. We were sensitive to $W_{\mathrm{r}}(2796) \geq 0.015 \AA$ in the $\mathrm{Q} 1634+706$ sight line only for $0.95 \leq z \leq 1.21$.
}

path. It has been customary to parameterize the distribution by either an exponential,

$$
n(W) d W=\left(\frac{N_{*}}{W^{*}}\right) \exp \left(-\frac{W}{W^{*}}\right) d W,
$$

or a power law,

$$
n(W) d W=C W^{-\delta} d W,
$$

where $N^{*}$ and $W^{*}$ (for the exponential) and $C$ and $\delta$ (for the power law) are parameters obtained by fitting the data. TBSYK fit the distribution with a power law of $\delta \sim 2$ for a sample with $W_{\mathrm{r}}^{\min }(2796)=0.25 \AA$ and mean redshift $\langle z\rangle \simeq 0.5$. For $W_{\mathrm{r}}^{\min }(2796)=0.3 \AA$ systems at $\langle z\rangle \simeq 1.6$, LTW fit the distribution to both an exponential and a power-law distribution [the latter in agreement with TBSYK]. LTW concluded that both adequately represented the data, with the exponential distribution slightly favored. SS92, who combined their data with that of SSB to obtain a very large homogeneous data sample of $\mathrm{Mg}$ II systems, found that $n(W)$ could be parameterized tolerably well by either an exponential, with $N^{*} \sim 1.5$ and $W^{*} \sim 0.66$, or by a power law, with $C \sim 0.4$ and $\delta \simeq 1.65$. SS92 noted that the exponential underpredicted the number of $W_{\mathrm{r}}(2796) \leq 0.5 \AA$ systems whereas the power law underpredicted the number with "intermediate" equivalent widths, those with $0.7 \leq W_{\mathrm{r}}(2796) \leq 1.3 \AA$.

Neither of the above functions given in Eqs. 7 and 8 account for evolution in the distribution with redshift, which is likely to be important when a large redshift range is being considered. TBSYK found evidence for more large equivalent width absorbers at high redshift than at low redshift. PB90 noted that the ratio of "weak" to "strong" absorbers, demarcated by $W_{\mathrm{r}}(2796)=0.6 \AA$, increased with decreasing redshift. With their large uniform sample, SS92 were capable of measuring how the number density evolution of $\mathrm{Mg}$ II systems changed as a function of the lower $W_{\mathrm{r}}(2796)$ cut off of their sample. As the limiting $W_{\mathrm{r}}(2796)$ is increased, the evolution becomes pronounced; large equivalent width systems evolve away with time. When $W_{\mathrm{r}}^{\min }(2796)=0.3 \AA$ is applied, the population of $\mathrm{MgII}$ absorbers is consistent with no-evolution expectations.

In Figure $6 \mathrm{a}$ and $\mathrm{b}$, we present $n\left(W_{\mathrm{r}}\right)$ for $W_{\mathrm{r}}(2796) \geq$ $0.0165 \AA$. We are $70 \%$ complete to this equivalent width. Solid triangles are the results from our survey. The data have been binned accounting for the 

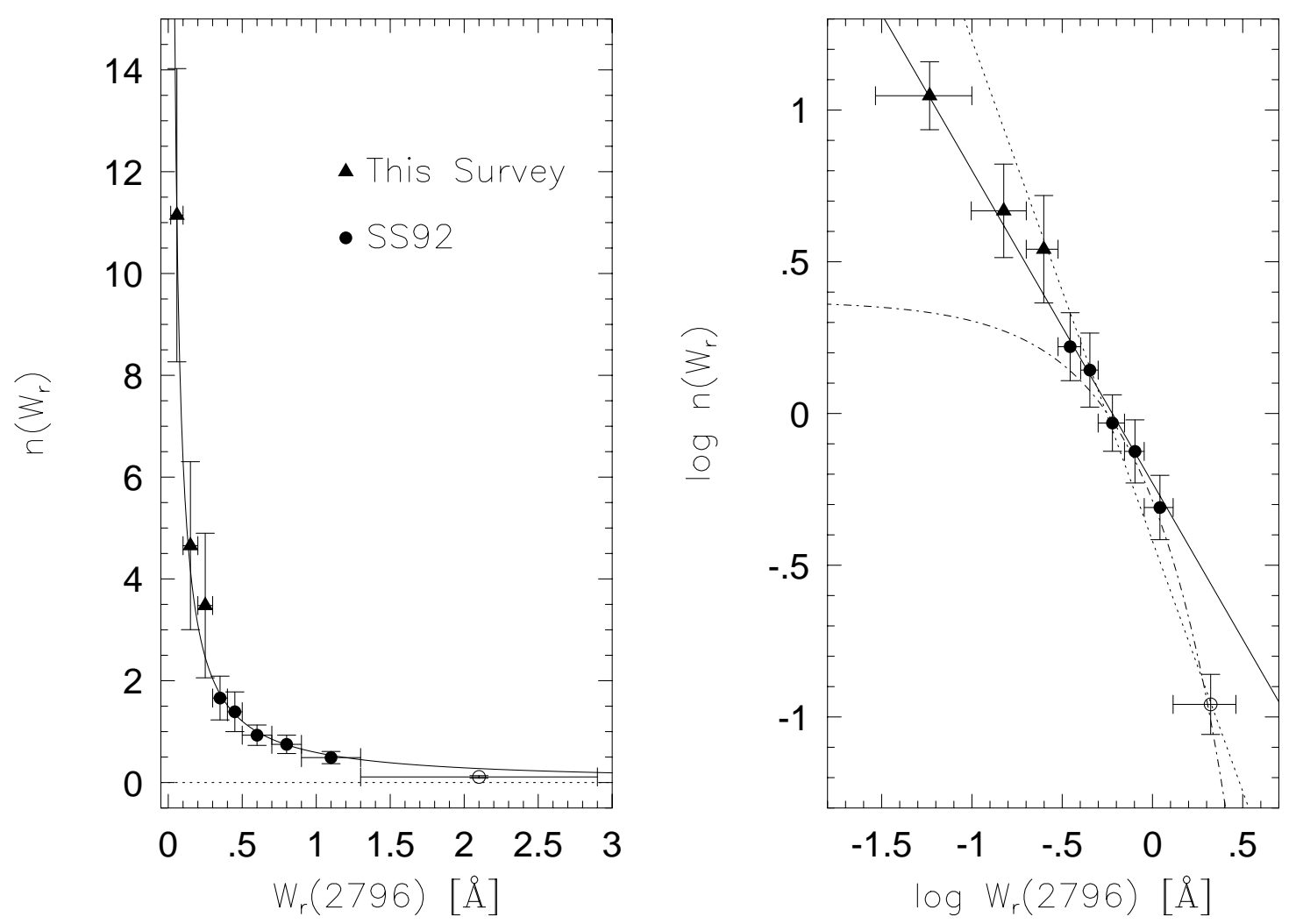

Fig. 6. - The equivalent width distribution verses the rest-frame equivalent width of the Mg II $\lambda 2796$ transition. The left hand panel illustrates the rapid increase in the number of small $W_{\mathrm{r}}(2796)$ systems. Solid triangles are from this study and solid circles are taken from SS92. The right hand panel shows a comparison of our power-law fit (solid line) to the combined data set from this study and SS92. Also shown are the fits presented by SS92 for a power law (dot-dot) and an exponential (dash-dot). The largest equivalent widths $\left[W_{\mathrm{r}}(2796) \geq 1.3 \AA\right.$; shown with an open dot] from the SS92 data are predominantly from redshifts higher than $z=1.4$, our upper cutoff. Due to the evolution of the strongest systems, this binned data point would be lower if the SS92 data were limited to our redshift coverage (see the text).

redshift path length over which the equivalent widths could have been detected. The three equivalent width bins are $[0.0165,0.1],[0.1,0.2]$, and $[0.2,0.3] \AA$ A. Shown as solid circles are the binned equivalent width data from SS92 (see their Figure 6), which span the range $0.3 \leq W_{\mathrm{r}}(2796) \leq 2.8 \AA$. Figure 6a illustrates the dramatic increase in $n\left(W_{\mathrm{r}}\right)$ with decreasing equivalent width. There is no turnover or break in the equivalent width distribution for $0.02 \leq W_{\mathrm{r}}(2796) \leq 0.3 \AA$ at $\langle z\rangle=0.9$.

The lack of a turnover is further illustrated in Figure $6 \mathrm{~b}$, which shows $\log n\left(W_{\mathrm{r}}\right)$ verses $\log W_{\mathrm{r}}(2796)$. The solid curve is a power law with $\delta=1.04$, which was obtained by minimizing the absolute deviation between the curve (Eq. 8) and the binned data. The absolute deviation for the presented fit is 0.07 (in $\log -$ $\log$ ). This fit is also presented in Figure 6a. It would be proper to fit the combined data of this survey and SS92 using the maximum likelihood technique employed by LTW, TBSYK, and SS92. This would require that we invoke the $g(W, z)$ function of SS92 and reanalyze the SS92 data over the redshift interval $0.4 \leq z \leq 1.4$. However, with this work, it is our intent to clearly demonstrate the absence of a turnover in the equivalent width distribution at small equivalent widths and to discern between the exponential and power-law parameterizations of the distribution. A reanalysis of the SS92 data was not required to demonstrate these points.

It appears that the evolution of the strongest systems, those with $W_{\mathrm{r}}(2796) \geq 0.6 \AA$, may have strongly biased the SS92 fits to the equivalent width distribution (a point they address in their paper). 
Slightly more than $60 \%$ of the equivalent widths 41 contributing to the largest bin $([1.3,2.8] \AA)$ taken from SS92 arise at $z \geq 1$.4. We have plotted this bin as an open circle in Figures 6a and 6b. If we had corrected this bin for our limited redshift coverage, it would be roughly $40 \%$ of its plotted value $(\log n \sim-1.46$, which is off the bottom of Figure $6 \mathrm{~b})$. There is no compelling evidence of such strong evolution in the equivalent width distribution for the remaining SS92 data $\left[0.3 \leq W_{\mathrm{r}}(2796) \leq 1.3 \AA\right]$. Thus, we have omitted the $[1.3,2.8] \AA$ bin from our fit, and include only the bins for which evolution is believed to be negligible.

To emphasize this point, we note that SS92 obtained a significantly steeper power law $(\delta=1.65)$ than the one we quote here $(\delta=1.04)$. This is likely due to the fact that the distribution of SS92 was fit over the full redshift range $0.2 \leq z \leq 2.2$, resulting in a bias from the relative paucity of large equivalent width systems at $z \leq 1.4$. This is illustrated in Figure $6 \mathrm{~b}$, in which we have plotted both the power-law fit (dot-dot) and the exponential fit (dash-dot) from SS92. Note that the $W_{\mathrm{r}}(2796) \leq 1.3 \AA$ data of SS92 also appear to be best described by the $(\delta=1.04)$ power law distribution. This possible bias in the SS92 fit provides a likely explanation for the trend of their exponential fit to underpredict the number of $W_{\mathrm{r}}(2796) \leq 0.5 \AA$ systems and for their power-law fit to underpredict the number with "intermediate" equivalent widths.

In summary, not only is there no turnover at small equivalent widths, but there is a strong break above $W_{\mathrm{r}}(2796) \sim 1.3 \AA$ at low redshifts, beginning around $1.0 \leq z \leq 1.5$. The upper limit on this slope is $\delta=-2.3$, for $W_{\mathrm{r}}(2796) \geq 1.0 \AA$ at $z \leq 1.4$. Nothing is known about the $n\left(W_{\mathrm{r}}\right)$ distribution function for $W_{\mathrm{r}}(2796) \leq 0.3 \AA$ above this redshift. A full analysis of the equivalent width distribution, including the redshift evolution as a function of equivalent width interval, would provide a more complete understanding of the evolution of $\mathrm{Mg}$ II absorbers out to $z \sim 2.2$. Such a study awaits the requisite high-resolution and high signal-to-noise ratio spectra covering $z \geq 1.4$.

${ }^{11}$ The estimate of $60 \%$ is based upon the observed numbers. Accounting for the relative number of sight lines observed by SS92 above and below $z=1.4$ would raise this estimate and accentuate our point.

\subsection{Clustering and the Issue of a Biased Sample}

The spectra used for this study are biased toward strong Mg II absorbers. If the weak Mg II systems tend to cluster around the stronger systems, then it is difficult to argue that the sample of weak systems is unbiased. On the other hand, if they do not cluster about the strong systems, we can conclude that the QSO sight lines are not biased toward an overabundance of weak systems.

We have computed the two-point velocity correlation function, which is presented in Figure 7, where the thick histogram distribution is the crosscorrelation function of weak systems with respect to the strong systems. There are no weak systems with velocity separations less than $1000 \mathrm{~km} \mathrm{~s}^{-1}$ from the strong ones, and there is no apparent signal in the velocity separations. To test if the weak systems are distributed like a random population with respect to the strong systems, we have computed the relative probability, $P(\Delta v)$, of detecting a $\Delta v$ separation from each strong absorption system. Following SS92,

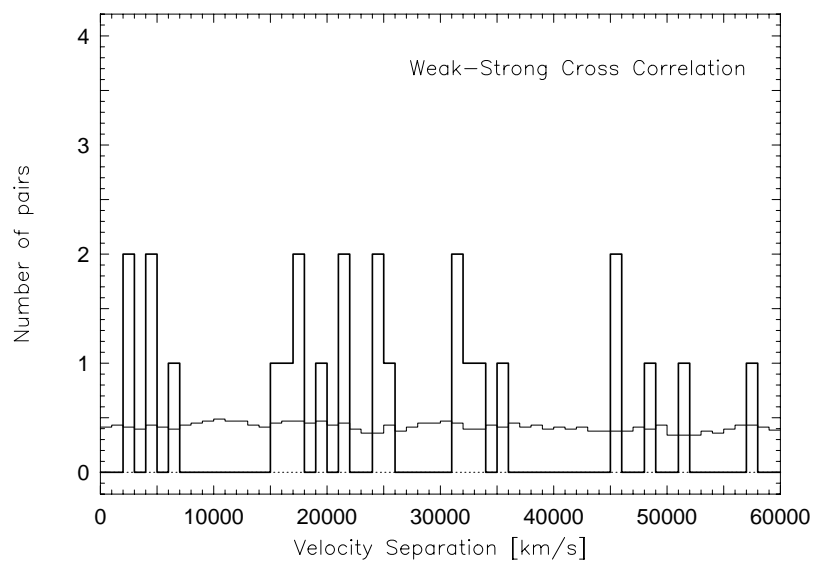

Fig. 7.- The two-point velocity cross-correlation function of weak and strong systems. Each bin gives the number of weak systems with velocity separations $\Delta v\left[\mathrm{~km} \mathrm{~s}^{-1}\right]$ from strong systems along the same QSO sight line. The expected number in each bin for a random distribution is given by the thin histogram. There is no evidence that the weak systems cluster in velocity about the strong systems. This implies that the QSO sight lines, though biased for the strong systems, are in fact unbiased for the presence of weak systems. 
we have limited our co-moving velocity difference to $\Delta v=60,000 \mathrm{~km} \mathrm{~s}^{-1}$, and have normalized the probability integral to the observed number of weak systems. Formally, the observed $\Delta v$ distribution is not inconsistent with a random distribution; a $\chi^{2}$ test on the binned data (1000 $\mathrm{km} \mathrm{s}^{-1}$ bins) yielded a probability of 0.18 that the two distribution were drawn from the same parent population.

We examined the redshift clustering of the weak systems with respect to one another and found that it is not inconsistent with a random distribution. The $\chi^{2}$ probability was 0.25 . These results suggest that the weak systems are statistically consistent with a random cosmological distribution. We conclude that the QSO sight lines surveyed are unbiased for the presence of weak MgII absorbers.

\subsection{Absorption Properties}

A more detailed examination of the cloud to cloud chemical and ionization conditions will be presented in Paper II. Here, we present the global absorption properties of the full systems. In Table 1 , we present the transitions that were covered for at least one of the 30 systems in our sample. The first three columns of the table are the ion and transition identity, the number of systems for which our spectra covered the transition, and the number of systems in which the transition was detected at the $3 \sigma$ level. The last two columns are the minimum equivalent width threshold ( $3 \sigma$ in the rest-frame) at which the transition was covered and the median for all systems in which it was covered. These are the strongest limits yet placed upon the majority of these transitions in Mg II selected absorption systems.

Of the transitions covered in the HIRES spectra, only $\operatorname{Mg} \mathrm{I} \lambda 2853$, FeII $\lambda 2344,2374,2383,2587$, and 2600 , and the AlIII $\lambda \lambda 1855,1863$ doublet were detected. The statistics are as follows: 13 of 29 have detected FeII (either $\lambda 2383$ or $\lambda 2600$ ), seven of 29 have detected MgI $\lambda 2853$ and each of these also has FeII, and three of four have detected AlıII. The $3 \sigma$ median equivalent width threshold for the FeII sample is $0.01 \AA$ for FeII $\lambda 2600$ and $0.008 \AA$ for FeII $\lambda 2383$. The threshold for $\mathrm{Mg} \mathrm{I}$ absorption is $0.006 \AA$ and for the AlIII doublet is $0.01 \AA$. The tight limits on NaI, FeI, CaII, and TiII trivially imply that, on average, absorbing gas selected by weak MgII absorption is not optically thick. If the ionization conditions and Hi column densities could be estimated, the CrII and ZnII limits could provide some clues to the mean metallicity and dust content of the absorbers.

Plotted in Figure 8 are the rest-frame Mg II $\lambda 2796$ equivalent widths as a function of redshift. There appears to be no trend in the distribution of weak absorbers with redshift. We have run SpearmanKendall (SK) non-parametric rank correlation tests to explore if any correlations are present among the detected absorption properties (limits were not included). We tested redshifts, velocity widths, equivalent widths, and doublet ratios against one another and found no correlations. The most suggestive ranking was an anti-correlation between $W_{\mathrm{r}}(2796)$ and the $\mathrm{Mg}$ II doublet ratio at the $1.4 \sigma$ level.

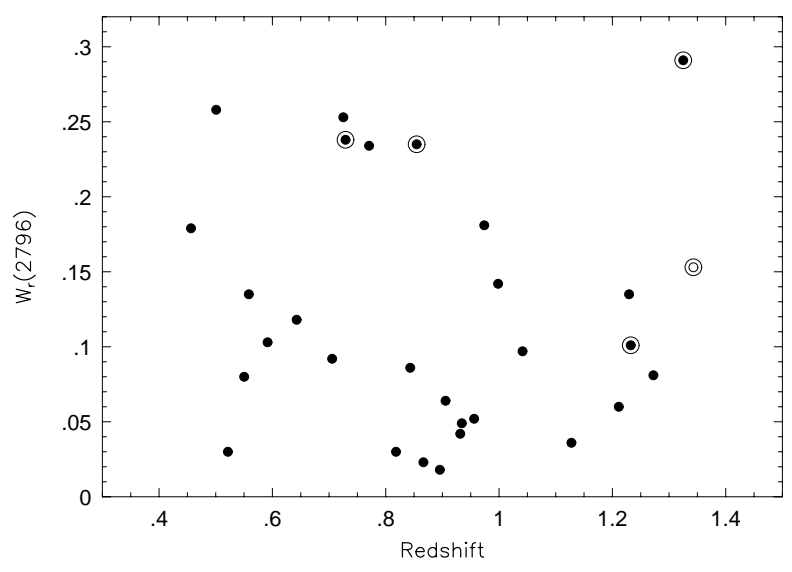

Fig. 8.- The full system rest-frame equivalent width of the Mg II $\lambda 2796$ transition verses the absorption redshift. There is no evidence of any trend in $W_{\mathrm{r}}(2796)$ with redshift. There is an indication that most all individual Mg II absorbing clouds likely have $W_{\mathrm{r}}(2796) \leq 0.15 \AA$. Those systems that are comprised of multiple absorption features are marked with a concentric circle; the individual "cloud" $W_{\mathrm{r}}(2796)$ are $\sim 0.15 \AA$ or less. The three remaining systems with $W_{\mathrm{r}}(2796) \sim 0.25 \AA$ have relatively poor signal-tonoise ratios; they are likely to be unresolved multiple clouds. See text for discussion.

\subsubsection{Individual Components}

As can be seen in Figure 8, most, if not all, of the individual clouds in these weak absorbers have $W_{\mathrm{r}}(2796) \leq 0.2 \AA$, and perhaps even $\leq 0.15 \AA$. The three $W_{\mathrm{r}}(2796) \geq 0.2 \AA$ systems, S2, S9, and S11, are likely comprised of two or more blended "clouds". In the case of S2, the profile clearly has multiple compo- 
nents. Voigt profile fits to these systems did in fact yield single components for each, but this is believed to be due to the relatively low signal-to-noise ratio in the spectra [based upon simulations (CVC98)].

Several of the systems (16\%) have multiple absorbing features. These systems are S10, S14, S27, $\mathrm{S} 29$, and S30. The detection of these multiple features is not correlated with the signal-to-noise ratio in the spectra, since they could be detected to $W_{\mathrm{r}}(2796)=0.02 \AA$ in $80 \%$ of the systems. Plotted in Figure 9 are the velocity widths, $\omega_{v}$, of the full MgII $\lambda 2796$ profiles as a function redshift. The dotted line at $\omega_{v}=2.46 \mathrm{~km} \mathrm{~s}^{-1}$, which is the Gaussian width of the instrumental profile, shows the threshold for fully unresolved features. Systems that have been resolved into multiple individual "clouds" are marked with a concentric circle. Note that the many single "cloud" systems are unresolved or only marginally resolved. The same holds true for the individual clouds in the multiple cloud systems. The average velocity width, $\omega_{v}$, of the individual "clouds" for the full sample is $\sim 4 \mathrm{~km} \mathrm{~s}^{-1}$. This small velocity width implies an average temperature of $\sim 25,000 \mathrm{~K}$. According to

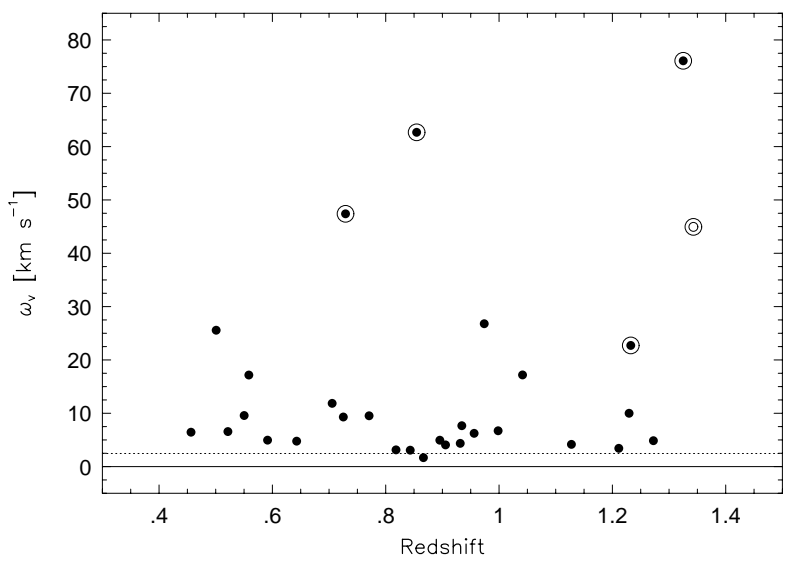

Fig. 9. - The velocity width, $\omega_{v}$ (equivalent Gaussian width), of the MgII $\lambda 2796$ transition as a function of absorption redshift. The dotted line gives the limit for unresolved absorption features. The data points with "rings" are those systems that have multiple absorption features separated by continuum. The open data point (with ring) is measured from the $\lambda 2803$ transition because the $\lambda 2796$ transition was unavailable. There is no evidence for any trend in $\omega_{v}$ with redshift.
Spearman-Kendall rank correlation tests, there are no trends in the overall (system) velocity widths with redshift over the interval $0.4 \leq z \leq 1.4$.

Of the seven systems in which MgI is detected, three have $W_{\mathrm{r}}(2796) \leq 0.11 \AA$. Two of the $\mathrm{MgI}$ "clouds" are in multiple component systems, S10 and S29, and these clouds have $W_{\mathrm{r}}(2796)=0.11 \AA$ and $0.05 \AA$, respectively. There does not appear to be a clear threshold for the presence of $\mathrm{MgI}$ with the equivalent width of $\mathrm{Mg}$ II down to $W_{\mathrm{r}}(2796) \sim 0.1 \AA$. It appears that MgI can survive in sub-LLS environments. In summary, we find that a typical individual MgII absorbing cloud is characterized by $W_{\mathrm{r}}(2796) \leq 0.2 \AA$ (or possibly $0.15 \AA$ ), and a temperature of $\sim 25,000 \mathrm{~K}$. The presence or non-presence of $\mathrm{Mg}$ I does not appear have a threshold dependence upon $W_{\mathrm{r}}(2796)$ down to $0.1 \AA$.

\subsection{Evolution}

It is plausible that, in addition to the observed increase in the number of the largest equivalent width absorbers at $z>1$, there might also be a paucity of weak absorbers at higher redshifts. If the absorbing gas is photoionized by the extra-galactic UV (EUV) background, then ionization conditions are expected to be higher at higher redshifts. In fact, Bergeron et al. (1994) have found that the ratio of "high" to "low" ionization metal-line absorbers increases with increasing redshift at a rate that is not inconsistent with a factor of $\sim 5$ increase in the EUV background (Kulkarni \& Fall 1993). Thus, it is interesting to investigate if we can place limits on the evolution of weak MgII systems at the higher redshifts we have surveyed. Though we had three sight lines (Q0958+551, Q1213-003, and Q1225+317) covering the redshift range $1.4 \leq z \leq 1.7$, we found no weak $\mathrm{Mg}$ II absorbers in this redshift interval.

Under the assumption of no evolution in the redshift path density of weak MgII absorbers, we can compute the expected number of absorbers for $1.4 \leq$ $z \leq 1.7$ and then compare that to our null detection rate. Normalizing to $d N / d z=1.74 \pm 0.1$ at $\langle z\rangle=0.9$ for the weak systems, we integrated Eq. 6 for nonevolution expectations $\left(\gamma=1\right.$ for $q_{0}=0$ and $\gamma=0.5$ for $q_{0}=0.5$ ). For both values of $q_{0}$, we obtained $N$ consistent with zero for our cumulative redshift path of $Z \sim 0.7$ over this redshift interval. Thus, our observations place no constraint on the evolution of the weak MgII absorbers over this redshift range. 
In principle, it might be that at $z \sim 2$ the $\mathrm{MgII}$ equivalent width distribution exhibits a cut off at the smallest equivalent widths. It is also expected that the power-law slope of the distribution is flatter for larger equivalent widths at $z \sim 2$ [there are more large $W_{\mathrm{r}}(2796)$ absorbers at high redshift] than that measured in this work at $z \sim 1$. This implies a very curious effect in the inferred evolution of MgII absorption strengths as the minimum equivalent width of the sample covering $0.4 \leq z \leq 2.2$ is continually decreased. It is well established that for $W_{\mathrm{r}}^{\min }(2796)=$ $0.3 \AA$, the population is consistent with no-evolution expectations, and that the lack of evolution is dominated by the lower end of the equivalent width distribution to this $W_{\mathrm{r}}^{\text {mnim }}(2796)$. When $W_{\mathrm{r}}^{\text {mnim }}(2796)$ is increased, the evolution becomes pronounced in that the ratio of "large" to "small" $W_{\mathrm{r}}(2796)$ absorbers increases with increasing redshift (SS92). Since the EUV background is more intense at higher redshifts, one would expect that the presence of Mg II is increasingly dependent upon photon shielding by neutral hydrogen; at $z \sim 2$ (and above), it would be expected that Mg II would not survive in sub-LLS environments (also see discussion in $\S 7$ of SS92). Therefore, as $W_{\mathrm{r}}^{\min }(2796)$ is decreased from $0.3 \AA$ to $\sim 0.02 \AA$ for a $0.4 \leq z \leq 2.2$ sample, evolution should again become apparent, this time due to a paucity of weak systems at the higher redshifts. Apparently, it may be only the $0.3 \leq W_{\mathrm{r}}(2796) \leq 1.3 \AA$ part of the MgII absorber population that exhibits no evolution (statistically) in redshift number density over $0.3 \leq z \leq 2.2$. This trend is tentatively suggested by the fact that at $\langle z\rangle=0.9$ there is a $30 \%$ difference between the $d N / d z$ of $W_{\mathrm{r}}^{\min }(2796)=0.3 \AA$ absorbers and LLS absorbers, whereas there is only an $8 \%$ difference at $\langle z\rangle=2$ (SS92; Stengler-Larrea et al. 1995).

\section{On the Nature of Weak Mg II Absorbers}

\subsection{Comparison with Lyman Limit Systems}

For the weak MgII absorbers, the redshift path density and lack of cosmological evolution are virtually indistinguishable from those of the population of LLS absorbers (SS92; see right hand panel of Figure 5). Moreover, a corresponding Lyman limit break is almost always found in UV spectra at the redshift of a strong MgII absorber (Lanzetta 1988). Based upon photoionization modeling (Bergeron \& Stasińska 1986; SS92), this had been attributed to the need for $\tau_{912} \geq 1$, which shields photons with energies above the ionization potential at which MgII can be destroyed. Thus, strong MgII absorbers are believed to arise in LLS environments. That is not to claim that all strong MgII absorbers are LLS absorbers; Bergeron et al. (1994) reported the existence of Mg II absorbers in sub-LLS environments at $z \sim 0.5$.

The above numbers are very constraining and we are led to conclude that virtually all weak MgII $a b$ sorbers arise in sub-LLS environments. This can be inferred directly from Figure 5 (right hand panel). The $d N / d z$ of $W_{\mathrm{r}}^{\min }(2796)=0.3 \AA \mathrm{Mg}$ II absorbers and of LLS absorbers are consistent within uncertainties, with the number density of the strong MgII absorbers being slightly higher (SS92; Stengler-Larrea et al. 1995). However, for the $W_{\mathrm{r}}^{\min }(2796)=0.02 \AA$ MgII absorbers (combined sample of this work and SS92), $d N / d z=2.65$, which is a factor of three to four greater than that of the strong MgII-LLS absorbers.

That weak MgII systems arise in sub-LLS environments is also consistent with the expectations for photoionized clouds. We have obtained preliminary Voigt profile fits of the MgII absorption profiles using the methods described in Churchill (1997a). We will present the full results in Paper II. From these fits, we have obtained fairly robust measurements of the Mg II column densities, which quasi-uniformly span the range $10^{11.8} \leq N\left(\mathrm{Mg}_{\mathrm{II}}\right) \leq 10^{13.2} \mathrm{~cm}^{-2}$. We then built a grid of photoionization models using CLOUDY (Ferland 1996), where we have assumed a Haardt \& Madau (1996) EUV background spectrum normalized at $z=1$ and a solar abundance pattern with $\left[Z / Z_{\odot}\right]=-1$ (which can be interpreted as the "gasphase metallicity", accounting for possible dust depletion of MgII). The model clouds are constant density plane-parallel slabs, each defined by its neutral hydrogen column density and ionization parameter, $U=n_{\gamma} / n_{\mathrm{H}}$, where $n_{\gamma}$ and $n_{\mathrm{H}}$ are the number density of photons capable of ionizing hydrogen and the total hydrogen number density, respectively.

In Figure 10, the grid of clouds is plotted for $N(\mathrm{Mg}$ II $)$ verses the total hydrogen column density, $N(\mathrm{HI})+N(\mathrm{HII})$. The molecular fraction, $f\left(\mathrm{H}_{2}\right)$, was always negligible. Solid curves are lines of constant $N(\mathrm{HI})$, and dotted curves are lines of constant $\log U$ presented at intervals of 0.5 dex. The range of observed weak MgII absorber column densities is crudely represented by the shaded region. Note that the majority of the absorbers are predicted to arise in sub-LLS clouds, those with $N(\mathrm{HI}) \leq 10^{17.3} \mathrm{~cm}^{-2}$. 


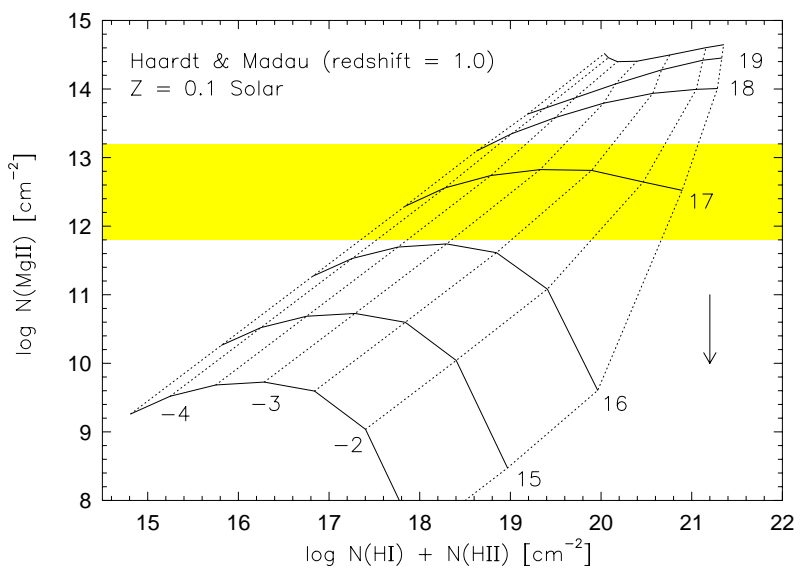

Fig. 10.- MgII column density verses total hydrogen column density for a grid of CLOUDY photoionization models, assuming a Haardt \& Madau (1996) UV flux at $z=1$. A $Z=0.1 Z_{\odot}$ abundance pattern has been assumed. The solid curves are contours of constant $N(\mathrm{HI})$ and the dot-dot curves are contours of constant ionization parameter, $\log U$. The shaded region gives the locus of measured $N\left(\mathrm{Mg}_{\mathrm{II}}\right)$ for the sample, based upon Voigt profile fits to the data (Paper II). For this abundance pattern, clouds which have $N($ FeII $) / N(\mathrm{Mg}$ II $) \sim 1$ must have low ionization, $\log U \sim-3.5$. The arrow shows how the grid would move if the metallicity of the models were decreased by 1 dex.

The main point to be gleaned from Figure 10, however, arises due to the metallicity dependence of the photoionization grid. If the metallicity of the model clouds were reduced by 1 dex, the entire grid would move approximately 1 dex downward along the $N(\mathrm{Mg}$ II $)$ axis. Thus, if the metallicity of the clouds were any lower than $\left[Z / Z_{\odot}\right]=-1$, the majority of the inferred neutral hydrogen column densities would lie above the Lyman limit value of $10^{17.3} \mathrm{~cm}^{-2}$. This is not allowed because most all weak MgII absorbers must arise in sub-LLS environments as constrained by the $d N / d z$ of LLS absorbers. In fact, from this grid, we can infer that most weak MgII absorbers are constrained to have metallicities $\left[Z / Z_{\odot}\right] \geq-1$. This argument applies for both low ionization and high ionization systems.

If the weak MgII absorbers are photoionized by a Haardt \& Madau-like spectrum, then the cloud metallicities are $(1)\left[Z / Z_{\odot}\right] \geq-1$ with $[\alpha / \mathrm{Fe}] \sim$ $[\alpha / \mathrm{Fe}]_{\odot}$, or $(2)\left[Z / Z_{\odot}\right]$ slightly below -1 with $[\alpha / \mathrm{Fe}]>$ $[\alpha / \mathrm{Fe}]_{\odot}$ by a factor of a few. Such abundance patterns are consistent with those observed in the Galaxy (Lauroesch et al. 1996; Savage \& Sembach 1996). The metallicity cannot typically be significantly lower than $\left[Z / Z_{\odot}\right]=-1$, since that would require an implausibly large enhancement of $[\alpha / \mathrm{Fe}]$ with respect to the solar ratio.

\subsection{Comparison with CIV Systems: Ioniza- tion Conditions}

Sargent, Boksenberg, \& Steidel (1988) found a $d N / d z$ of $1.76 \pm 0.33$ for C IV at $\langle z\rangle=1.5$ for a sample complete to $W_{\mathrm{r}}^{\min }(1548)=0.3 \AA$. We note that this value is consistent with the number per unit redshift of the weak $\mathrm{Mg}$ II systems $\left[0.02 \leq W_{\mathrm{r}}(2796) \leq 0.3 \AA\right.$ at $\langle z\rangle=0.9]$. Does this imply that the population of systems selected by weak MgII absorption are, in essence, the same population as selected by the presence of CIV with $W_{\mathrm{r}}^{\min }(1548)=0.3 \AA$ ?

It is not expected that all weak MgII systems at $\langle z\rangle=0.9$ would have associated CIV with $W_{\mathrm{r}}(1548) \geq$ $0.3 \AA$, since the CIV number density is seen to decrease with decreasing redshift for this equivalent width threshold. Bergeron et al. (1994) found $d N / d z=$ $0.87 \pm 0.43$ for CIV with $W_{\mathrm{r}}^{\min }(1548)=0.3 \AA$ at $\langle z\rangle=0.3$. We have confirmed this expectation from reports in the literature and from a preliminary, yet semi-rigorous, search through the FOS/HST spectra of Dobrzycki et al. (1998) using their search and detection software (with the kind assistance of A. Dobrzycki). We conclude (see $\$ 3.1 .2$ ) that roughly half of the weak MgII systems would have detectable associated CIV to the $\sim 3 \sigma$ significance level in spectra available in the literature and in the HST Archive, where the $3 \sigma$ equivalent width detection threshold ranged from $\sim 0.15$ to $0.3 \AA$ in the HST spectra.

In and of itself, CIV absorption is an important indicator of the ionization level in absorbers selected by weak MgII absorption. A more powerful indicator, however, is the relative absorption strengths of $\mathrm{CIV}$ and FeII. In the upper panels ( $\mathrm{a}$ and $\mathrm{b}$ ) of Figure 11, we present the column densities of MgII, FeII, MgI, AlıII, and CIV as a function of ionization parameter for CLOUDY (Ferland 1996 models. We have assumed $N(\mathrm{HI})=10^{16.5} \mathrm{~cm}^{-2}$ for two metallicities, $\left[Z / Z_{\odot}\right]=-1$ and 0 , respectively, with a Haardt \& Madau (1996) UV background flux normalized at $z=1$. The bottom panels (c and d) show the restframe equivalent widths for a Doppler parameter of 

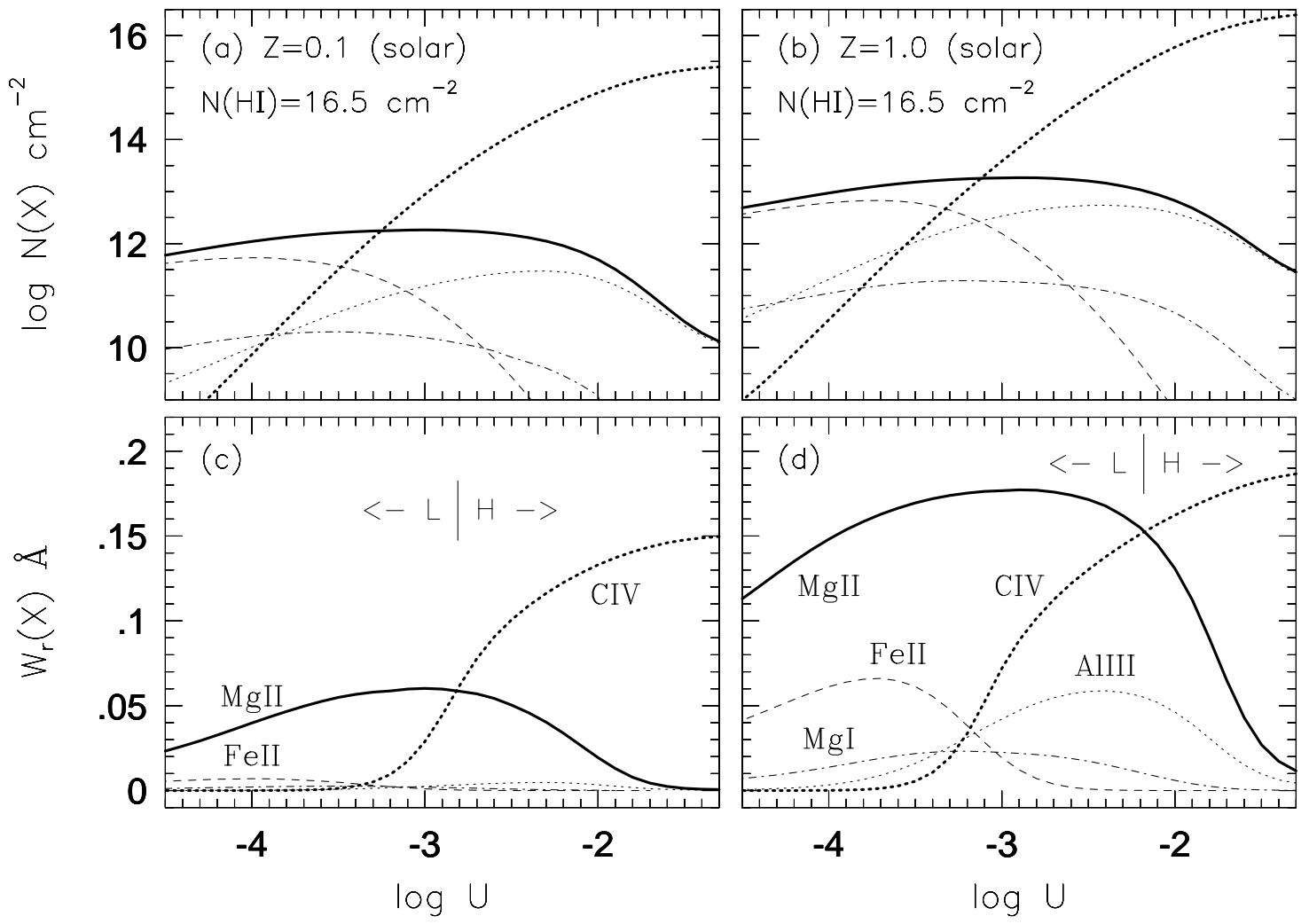

Fig. 11.- Photoionization models with $N(\mathrm{HI})=10^{16.5} \mathrm{~cm}^{-2}$ for solar abundance patterns with $\left[Z / Z_{\odot}\right]=-1$ (left panels) and $\left[Z / Z_{\odot}\right]=0$ (right panels). The upper panels show the observed column densities as a function of ionization parameter and the lower panels show the rest-frame equivalent widths for $b=6 \mathrm{~km} \mathrm{~s}^{-1}$ for CIV $\lambda 1548$, MgII $\lambda 2796$, FeII $\lambda 2600, \operatorname{Mg}$ I $\lambda 2853$, and AliII $\lambda 1855$. Low and high ionization ("L" and "H", respectively) are defined by the ratio of $W(\mathrm{CIV}) / W(\mathrm{Mg} I \mathrm{I})$.

$b=6 \mathrm{~km} \mathrm{~s}^{-1}$, the median velocity width of the individual MgII absorption components 12 .

The models presented have MgII $\lambda 2796$ equivalent widths consistent with the observed range for individual components (see Figure 8 and discussion in \$.4.1). For these sub-LLS clouds, the models show no temperature or ionization structure with cloud depth; the kinetic temperature is the same for all ionization species. For the modeled column densities, $\operatorname{Mg}$ I $\lambda 2853$, FeII $\lambda 2600$, and AlIII $\lambda 1855$ are on the linear part of the curve of growth ( $b$ independent). For MgII $\lambda 2796$ and CIV $\lambda 1548$, the value of the $b$ parameter becomes important for column densities greater than $\sim 10^{11} \mathrm{~cm}^{-2}$ and $\sim 10^{13} \mathrm{~cm}^{-2}$, respec-

${ }^{12}$ Inspection of the $\mathrm{Mg}$ II profiles with $\omega_{v} \geq 10 \mathrm{~km} \mathrm{~s}^{-1}$ (for example, S2, S4, S5, S21, S23) reveals that they are blends of narrower components. Preliminary Voigt Profile decomposition of the profiles reveals this to be true and that the widths are smaller than or consistent with $b=6 \mathrm{~km} \mathrm{~s}^{-1}$. tively. We have neglected thermal scaling of the $b$ parameters, which would yield slightly elevated CIV equivalent widths (a $30 \%$ increase at $10^{16} \mathrm{~cm}^{-2}$ ).

The demarcation between low and high ionization absorbers ( $\mathrm{L}$ and $\mathrm{H}$, respectively, as marked on panels $\mathrm{c}$ and $\mathrm{d}$ of Figure 11) is given by the ratio $W_{\mathrm{r}}(\mathrm{CIV} \lambda 1548) / W_{\mathrm{r}}(\mathrm{Mg}$ II $\lambda 2796)$, where greater than unity designates high ionization (Bergeron et al. 1994). For these single phase photoionization models, high ionization clouds have $N($ FeII $) \ll N\left(\mathrm{Mg}_{\mathrm{II}}\right)$ and $N$ (FeII) $\ll N(\mathrm{CIV})$. For low ionization gas there is a range of $N$ (FeII) as compared to $N$ (CIV) and $N(\mathrm{Mg} \mathrm{II})$. For very low ionization conditions, $N$ (FeII) can be comparable to $N(\mathrm{Mg} I \mathrm{I})$, with both much larger than $N$ (Civ). Thus, we would not expect CIV to be reported in the literature nor found in FOS/HST spectra for clouds that roughly have $W_{\mathrm{r}}(\mathrm{Fe}$ II $\lambda 2600) / W_{\mathrm{r}}(\mathrm{Mg}$ II $\lambda 2796)>0.1$. In fact, for no case in which we have detected FeII would we expect high ionization as defined by $W_{\mathrm{r}}(\mathrm{C}$ IV $\lambda 1548) /$ 
$W_{\mathrm{r}}(\operatorname{Mg}$ II $\lambda 2796) \geq 1$. With regard to detectable absorption, the point is that the presence of FeII absorption at a non-negligible level is a strong indicator of low ionization conditions for single phase clouds. The above arguments remain valid for $N(\mathrm{HI})=10^{17} \mathrm{~cm}^{-2}$ cloud models in which some ionization structure is present, resulting in roughly constant $N$ (MgII) as a function of $\log U$, but identical behavior in $N$ (FeII) and $N(\mathrm{CIV})$.

In terms of $3 \sigma$ detections, of the 18 systems for which CIV and FeII was available, three have FeII but no CIV (S7, S10, and S18). These may be low ionization systems. Five of the 18 systems have CIV but no FeII (S5, S17, S19, S20, and S23). Given the constraint $\left\langle W_{\mathrm{r}}^{\min }(\right.$ Fe II $\left.\lambda 2600)\right\rangle=0.01 \AA$ (see Table đ), these are likely to be high ionization systems. Four of the 18 systems have both CIV and FeII (S14, S28, S29, and S30), two of which (S28 and S29) have CIV, FeII, and $\mathrm{Mg}$ I. The simultaneous presence of FeII and CIV implies that a single cloud or a single phase absorption model is not adequate. Overall, it appears that the population of metal-line absorbers selected by weak Mg II absorption have a range of ionization conditions, possibly including multi-phase conditions.

\subsection{Multi-Phase Ionization?}

From Figure 11, we see that it is virtually impossible to obtain $W_{\mathrm{r}}(\mathrm{CIV} \lambda 1548)>0.2 \AA$ for single-phase photoionization models with profile widths (i.e. $b$ parameters) consistent with those observed for individual MgII components. This is because of the very flat dependence of $W_{\mathrm{r}}(1548)$ on $N(\mathrm{CIV})$ for the small $b$ parameters implied by the individual MgII components; the upper limit holds for higher $N(\mathrm{HI})$ cloud models. The upshot is that if $W_{\mathrm{r}}(1548)$ is significantly greater than $\sim 0.2 \AA$ in a single component absorber with $W_{\mathrm{r}}(2796) \leq 0.2 \AA$, one of two possibilites can be inferred: (1) Either the absorber has multiple ionization phases, such that the MgII arises in a cooler low-ionization region embedded in a higher ionization medium, or (2) additional multiple components (spatially distinct) are observed in CIV but not in MgII, in which case a high resolution spectrum of the CIV profile would be required to determine the ionization level of the component seen in MgII.

Three of the four systems exhibiting both CIV and FeII absorption, S14, S29, and S30, have multiple unblended components spread over $\sim 100 \mathrm{~km} \mathrm{~s}^{-1}$. It is quite plausible that they are multi-phase, that low ionization MgII components are embedded in a high ionization "halo". Considering S29, we estimate $W_{\mathrm{r}}(1548) \simeq 0.9 \AA$ (lower limit) from the unresolved CIV equivalent width reported by SS92. For $N(\mathrm{HI}) \leq 10^{17} \mathrm{~cm}^{-2}$, the CIV equivalent width could be produced as the component sum of high ionization clouds (near-solar metallicity required), each component contributing $\sim 0.2 \AA$ to the total CIV equivalent width. However, this is unlikely because the FeII absorption enforces low ionization conditions in the phase giving rise to MgII absorption. The mean equivalent width of the individual MgII $\lambda 2796$ components is $0.05 \AA$ and that of FeII $\lambda 2600$ is $0.015 \AA$. Refering to Figure 11, the mean ratio of $W_{\mathrm{r}}(2600) / W_{\mathrm{r}}(2796) \simeq 0.3$ for $W_{\mathrm{r}}(2796) \simeq 0.05 \AA$ implies ionization parameters in the range $-3.5 \leq$ $\log U \leq-4$; these are low ionization clouds. Thus, the overall absorption properties of S29 are more consistent with low ionization MgII components embedded in a high ionization medium or juxtaposed with additional spatially distinct high ionization components.

For the strong Mg II absorbers, preliminary results from a study incorporating $R=1300$ data of the high ionization species (esp. the CIV doublet) indicate a strong correlation between the CIV equivalent width and the kinematic spread of the MgII absorbing gas (Churchill 1998). This correlation is much less significant for the MgII equivalent width with its own kinematic spread. Furthermore, based upon 11 absorbing galaxies, there is no apparent ionization structure in the halos in that $W_{\mathrm{r}}(\mathrm{CIV}) / W_{\mathrm{r}}(\mathrm{Mg}$ II $)$ does not correlate with the QSO-galaxy impact parameter (i.e. projected galactocentric distance). These preliminary findings may indicate a hot pressure confining halo or extended layer of gas around these galaxies that may be providing an environment in which weak Mg II absorbing clouds could survive at large galactocentric distances (a multi-phase environment?). Because of the large intrinsic scatter in absorption properties as a function of galaxy properties (Churchill et al. 1996), the number of galaxies studied thus far is too small to be conclusive about the supposition of multi-phase ionization in the outskirts of galaxies.

\subsection{The Physical Sizes of the Absorbers}

The measured $d N / d z$ can be used to constrain several properties of the absorbers, in particular their physical sizes. Here, we assume that all absorbers are associated with luminous galaxies (cf. Churchill et al. 1996; Steidel 1995). However, without direct knowledge of the galaxies and their absorbing gas proper- 
ties, we can constrain only the product of the population cross section, $\sigma$, and number density $n$.

First, the galaxy luminosity function is required to give the number of galaxies per unit luminosity per unit co-moving volume. For a Schechter function (1976), $\Phi(L) d L=\Phi^{*}\left(L / L^{*}\right)^{\alpha} \exp \left(-L / L^{*}\right) d L$, there are two important parameters: the faint-end slope, $\alpha$, and the density of $L^{*}$ galaxies, $\Phi^{*}$. Second, a Holmberg (1975) dependence, $R(L)=R_{*}\left(L / L^{*}\right)^{\beta}$, of the spatial extent of the absorbing gas on the luminosity of the galaxy is assumed, where $R_{*}$ is the fiducial size of an $L^{*}$ galaxy. The product $\sigma n$, is given by $\sigma n=\pi \int_{L_{\min }}^{\infty} \Phi(L) R^{2}(L) d L$, which simplifies to

$$
\sigma n=\pi \Phi^{*} R_{*}^{2} \Gamma\left(\alpha+2 \beta+1, L_{\min } / L^{*}\right),
$$

where $\Gamma$ is the incomplete gamma function, which accounts for the additional assumption of a minimum luminosity, $L_{\min }$, at which a galaxy will no longer exhibit Mg II absorption to a well-defined detection level at any impact parameter. Assuming no evolution in the redshift path density of absorbing galaxies, the relation between $\sigma n$ and the measured $d N / d z$ is

$$
\frac{d N}{d z}=\frac{c \sigma n}{H_{0}}(1+z)\left(1+2 q_{0} z\right)^{-1 / 2} .
$$

For $W_{\mathrm{r}}^{\min }(2796)=0.3 \AA$, Steidel (1995) directly measured $\beta=0.15$ and $R^{*}=38 h^{-1} \mathrm{kpc}$ for the Holmberg relation and $L_{\min } / L^{*} \sim 0.06$ for $K$-band luminosities. From this, Steidel et al. (1994) deduced a faint-end slope of $\alpha=-1$ and a number density of $\Phi^{*}=0.03 h^{3} \mathrm{Mpc}^{-3}$ for galaxies selected by the presence of MgII absorption. These values are in good agreement with the values $\alpha=-0.9$ and $\Phi^{*}=0.033 h^{3} \mathrm{Mpc}^{-3}$ measured for the full CFRS sample of galaxies over the similar redshift range of $0.0 \leq z \leq 1.3$ (Lilly et al. 1995).

However, it is not entirely clear that the radiusluminosity relationship measured by Steidel (1995) is applicable when the equivalent width detection threshold is lowered to $W_{\mathrm{r}}^{\min }(2796)=0.02 \AA$. As mentioned above, the strong Mg II systems are closely associated with LLS absorbers, whereas the majority of weak systems must be arising in sub-LLS absorbers. The apparently sharp cut off of MgII absorbing "halos" at $\sim 40 h^{-1} \mathrm{kpc}$ implies a sharp edge in the neutral hydrogen column density at $N(\mathrm{HI}) \sim$ $10^{17.3} \mathrm{~cm}^{-2}$ that is luminosity dependent $(\beta \neq 0)$. Indeed, such rapid declines in neutral hydrogen have been seen in $21 \mathrm{~cm}$ maps of local galaxies (Corbelli,
Schneider, \& Salpeter 1989; van Gorkom et al. 1993). However, there is evidence that the decline slows at larger radii, such that sub-LLS gas may extend to a significantly greater distance. Bowen, Blades, \& Pettini (1995) found that the $\beta$ dependence of $R(L)$ for Hi became weaker $(\beta$ decreased) for smaller $N(\mathrm{HI})$ thresholds and that $\beta$ approached the value measured for $\mathrm{Mg}$ II systems when $17 \leq N(\mathrm{HI}) \leq 18 \mathrm{~cm}^{-2}$. If this trend continues as $N(\mathrm{HI})$ decreases below the Lyman limit, then, in principle, the dependence of $R(L)$ on $\beta$ could vanish or be very weak for sub-LLS conditions.

The whole approach to inferring the absorber sizes could be complicated by the fact that more than one population of luminous object may be associated with the absorbing gas. As suggested by Churchill \& Le Brun (1998), it is possible that low surface brightness (LSB) galaxies may contribute to the measured $d N / d z$ of weak MgII absorbers 13 . At redshift $z \sim 0$, Dalcanton et al. (1997) has measured $\Phi^{*}=$ $0.08 h^{3} \mathrm{Mpc}^{-3}$ for LSB galaxies with central surface brightnesses of $23 \leq \mu_{0}(V) \leq 25 \mathrm{mag} \operatorname{arcsec}^{-2}$. This is a factor of $\sim 2.5$ higher than the number density of galaxies exhibiting the Freeman (1970) central surface brightness of $\mu_{0}(B)=21.7 \mathrm{mag} \operatorname{arcsec}^{-2}$, which is characteristic of the $W_{\mathrm{r}}(2796)>0.3 \AA \mathrm{Mg}$ II selected galaxies. Thus, we cannot simply assume that the luminosity function of galaxies selected by weak Mg II absorption would have the same co-moving number density as that found by Steidel et al. (1994). Moreover, there is no a priori reason to assume that the $L_{\min } / L_{K}^{*}=0.06$ cut off should apply for a population of galaxies selected by $W_{\mathrm{r}}(2796)<0.3 \AA$ absorption.

For $W_{\mathrm{r}}^{\min }(2796)=0.02 \AA$, we found $d N / d z=2.71$ at $\langle z\rangle=0.9$. Writing Eq. 9 to obtain $R_{*}$ as a function of $\Phi^{*}, L_{\min } / L^{*}, \alpha$, and $\beta$, gives,

$$
R_{*}=\frac{79.5 h^{-1} \mathrm{kpc}}{\left[\left(\Phi^{*} / \Phi_{(\mathrm{CFRS})}^{*}\right) \Gamma\left(\alpha+2 \beta+1, L_{\min } / L^{*}\right)\right]^{1 / 2}}
$$

where it is assumed that the absorbing gas is spherically distributed and has unity covering factor ${ }^{14}$.

${ }^{13}$ We comment that is interesting that LSB galaxies have not yet been identified with strong Mg II absorption, except possibly in the cases of a few damped Ly $\alpha$ systems.

${ }^{14}$ If one assumes that only some fraction, $f_{\mathrm{abs}}$, of the galaxies contribute to absorption, and that the gas covering factor, $f_{\mathrm{c}}$, is less than unity, then the quoted values of $R_{*}$ should be scaled by $\left(f_{\text {abs }} f_{\mathrm{c}}\right)^{-1 / 2}$. If one assumes the geometry is somewhat flattened, then an additional factor of $\kappa^{-1 / 2}$ can be introduced, 


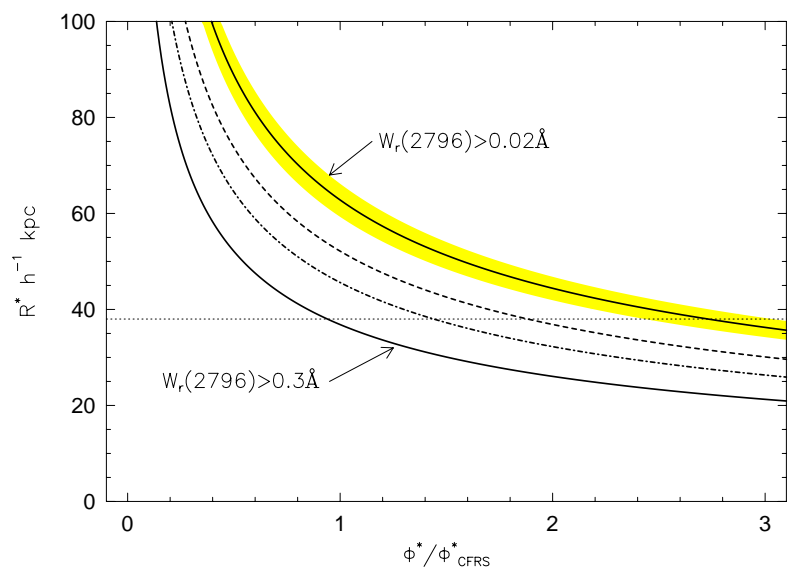

Fig. 12. - The inferred physical extent of MgII absorbing gas surrounding an $L^{*}$ galaxy verses $\Phi^{*} / \Phi_{\text {CFRS }}^{*}$, where $\Phi^{*}$ is the normalization of the galaxy luminosity function and $\Phi_{\text {CFRS }}^{*}$ is that of the CanadaFrance Redshift Survey (Lilly et al. 1995). Spherical geometry and unity covering factor have been assumed. The shaded region shows the spread due to the uncertainty, $\Delta \alpha= \pm 0.1$, in the faint-end slope of the luminosity function. The dashed curve is for the case $\beta=0$, which assumes no scaling in the gas cross section with galaxy luminosity. The dash-dot curve is for $L_{\min }=0$, which assumes there is no minimum lun inosity at which the Mg II absorbing gas cuts off. See the text for our discussion.

In Figure 12, we have plotted $R_{*}$ as a function of $\Phi^{*} / \Phi_{\mathrm{CFRS}}^{*}$, where $\Phi_{\mathrm{CFRS}}^{*}$ is the Schechter function normalization measured by Lilly et al. (1995) for $0.0 \leq$ $z \leq 1.3$ high surface brightness galaxies. The solid curve labeled " $W_{\mathrm{r}}(2796)>0.3 \AA$ " gives the radial extent of MgII absorbing gas above this equivalent width in an $L_{K}^{*}$ galaxy. The assumed parameters are those measured by Steidel (1995) and Steidel et al. (1994), namely $L_{\min } / L_{K}^{*}=0.06, \alpha=-1$, and $\beta=0.15$. Note that $R^{*}$ of $\Phi^{*} / \Phi_{\mathrm{CFRS}}^{*}=0.91$ is $38 h^{-1} \mathrm{kpc}$, as measured by Steidel (1995). It is quite remarkable that $\Phi^{*} / \Phi_{\text {CFRS }}^{*} \sim 1$ for these systems, given the abundance of LSB galaxies that could potentially contribute to strong Mg II absorption (Impey $\&$ Bothun 1989; Dalcanton et al. 1997).

The solid curve labeled " $W_{\mathrm{r}}(2796)>0.02 \AA$ " is computed assuming the same $L_{\min } / L^{*}, \alpha$, and $\beta$ as measured for $W_{\mathrm{r}}(2796)>0.3 \AA$ absorption selected

where $\kappa=0.5$ for infinitely thin "disks". galaxies, but with $d N / d z=2.65$. For $\Phi^{*} / \Phi_{\text {CFRS }}^{*} \sim 1$, the inferred $R_{*}$ is $63 h^{-1} \mathrm{kpc}$. If LSB galaxies have the same absorbing properties as do those observed by Steidel (1995), then the combined galaxy population would have $\Phi^{*} / \Phi_{\text {CFRS }}^{*} \sim 3$ (Dalcanton et al. 1997), which yields $R_{*} \sim 35 h^{-1} \mathrm{kpc}$. This is surprisingly consistent with the $R_{*}$ measured by Steidel (1995) for $W_{\mathrm{r}}(2796)>0.3 \AA$ absorption selected galaxies.

We now investigate the impact of our uncertainties in and assumptions about $L_{\min } / L^{*}, \alpha$, and $\beta$, as applied to the $W_{\mathrm{r}}(2796)>0.02 \AA$ sample. The shaded region around the " $W_{\mathrm{r}}(2796)>0.02 \AA$ " curve gives the spread in $R^{*}$ for a $\Delta \alpha= \pm 0.1$ uncertainty in the faint-end slope (Lilly et al. 1995). A larger $\alpha$ yields smaller $R^{*}$. The dot-dash curve for $W_{\mathrm{r}}(2796)>$ $0.02 \AA$ absorbers gives $R^{*}$ for $L_{\min } / L^{*}=0$ with $\alpha$ and $\beta$ unchanged. This illustrates the scenario in which there is no minimum luminosity, or galaxy mass, dependence on $R_{*}$. For the CFRS normalization, this gives $R^{*}=46 h^{-1} \mathrm{kpc}$. If $\beta \sim 0$ with $L_{\min } / L^{*}=0.06$ and $\alpha=-1$, then $R_{*}$ is reduced relative to a $\beta=0.15$ dependency, as shown with the dash-dot curve. If $\beta=0.4$ (the historically invoked "Holmberg" value), then $R^{*}=77 h^{-1} \mathrm{kpc}$ for $\Phi^{*} / \Phi_{\mathrm{CFRS}}^{*}=1$. In the unrealistic limit that all $0.02 \leq W_{\mathrm{r}}(2796) \leq 0.3 \AA \mathrm{Mg}$ II absorption arises in LSB galaxies, then $R_{*}=33 h^{-1} \mathrm{kpc}$ [uses $d N / d z=1.71$ and $\Phi^{*} / \Phi_{\text {CFrS }}^{*} \sim 2.5$ (Dalcanton et al. 1997)]. The upshot is that there is a great deal of uncertainty in what we can infer about the physical extent of MgII absorbers due to our almost complete lack of knowledge of how $W_{\mathrm{r}}(2796)<0.3 \AA \mathrm{Mg}$ II absorption properties depend (or do not depend) upon galactic properties.

\subsection{Objects Selected by Weak Mg II Absorp- tion}

Given the uncertainty in the inferred sizes of the absorbers, it is quite difficult to venture a definitive interpretation of what star forming objects are giving rise to the weak MgII absorbing gas. The question remains; what luminous objects could be associated with the large numbers of weak MgII systems at $z \sim 1$ and below? We point out that the two weak systems (S6 and S10) studied by Churchill et al. (1996) are massive (1.3 and $\left.3.7 L_{K}^{*}\right)$, red $(B-K>4)$, and at relatively large impact parameters (24 and $\left.36 h^{-1} \mathrm{kpc}\right)$. However, the situation overall is not so clear.

It is possible that weak MgII absorption arises in the extended regions of the well-studied population of Mg II absorbing galaxies out to large impact parame- 
ters (a region of $40 h^{-1} \leq R^{*} \leq 65 h^{-1} \mathrm{kpc}$, assuming $\Phi^{*} / \Phi_{\text {CFRS }}^{*}=1$ and depending upon the accepted values of $\alpha, \beta$, and $L_{\min } / L^{*}$ for $0.02 \leq W_{\mathrm{r}}(2796)<$ $0.3 \AA$ ). It is not unreasonable to suggest that some fraction of the Ly $\alpha$ lines seen to arise in the outskirts (beyond $\sim 40 \mathrm{kpc}$ ) of large extended halos or disks of galaxies (Lanzetta et al. 1995; Le Brun, Bergeron, \& Boissé 1996, Charlton \& Churchill 1996) may give rise to weak MgII absorption. It this may be that this would preferentially happen in the most massive galaxies, as seen for S6 and S10 (see Charlton \& thurchill 1998).

An alternative possibility is that a substantial fraction of the weak MgII population is selecting a high metallicity tail of the sub-LLS environments around LSB galaxies or low luminosity galaxies. If the absorbing gas is associated with LSB galaxies, the value of $\Phi^{*} / \Phi_{\text {CFRS }}^{*}$ for the number of Mg II absorbing galaxies would be increased, since two "populations" of Mg II absorbing galaxies would be contributing to its value. That is, some fraction of weak systems could arise in "normal" high surface brightness galaxies (perhaps at greater impact parameters) and some fraction could arise in LSB galaxies. A total of 18 of the weak Mg II systems are in QSO fields for which the redshifts of the galaxies have been measured out to 10 or more arcsec (Steidel, private communication). This corresponds to impact parameters of $\sim 40 h^{-1} \mathrm{kpc}$ at $z=0.5$ to $\sim 55 h^{-1} \mathrm{kpc}$ at $z=1.0$. Only four of the 18 have identified associated galaxies (though some have plausible candidates; see $\$ 3.2$. . Most, but not all, QSO fields have only one or two galaxies within 20 arcsec and these are often identified with strong Mg II systems. Thus, for a fair number of the weak systems, it may be that undetected low luminosity dwarf or LSB galaxies at impact parameters $\leq 50 h^{-1}$ are giving rise to the absorption.

Other possibilities include (1) isolated star forming or post star forming dwarf galaxies and/or pre-galaxy fragments (Yanny \& York 1992), (2) tidally disrupted gas from past galaxy-galaxy events (Bowen et al. 1995), or (3) large associations of sub-LLS clouds clustered with the over densities surrounding formed galaxies (Le Brun et al. 1996). However, the null result in the velocity cross-correlation of weak and strong absorbers is not suggestive of the latter two hypotheses.

\section{Summary}

We searched for weak Mg II $\lambda \lambda 2976,2803$ doublets, those with rest-frame equivalent widths $W_{\mathrm{r}}(2796) \leq$ $0.3 \AA$, in HIRES/Keck spectra of 26 QSOs. The QSO sight lines are unbiased for these weak systems. The cumulative redshift path was $Z \sim 17$ over the range $0.4 \leq z \leq 1.4$ and $Z \sim 0.7$ for $1.4 \leq z \leq 1.7$. The survey was complete to $W_{\mathrm{r}}(2796)=0.06 \AA$, and $80 \%$ complete to $W_{\mathrm{r}}(2796)=0.02 \AA$, where we have enforced a $5 \sigma$ detection limit. A total of 30 systems were detected, of which 23 were discovered in these spectra. The MgI $\lambda 2853$ transition was detected in seven of the systems and FeII, especially the $\lambda 2600$ and/or the $\lambda 2383$ transition, was detected in half of the systems. When AlIII $\lambda \lambda 1855,1863$ was covered, we found it in three of four systems. Based upon a literature search and a search through the FOS/HST spectra of Dobryzycki et al. (1998), we found CIV was detected in roughly half of the systems to $3 \sigma$ equivalent width threshold of 0.15 to $0.3 \AA$ (rest-frame). No systems were found at $1.4 \leq z \leq 1.7$, though this is consistent with expectations when we extrapolate from lower $z$, given that the cumulative redshift path was only $Z \sim 0.7$ over this redshift range.

We have combined our sample with the $W_{\mathrm{r}}(2796) \geq$ $0.3 \AA$ sample MG1 of SS92, taken over the redshift interval $0.4 \leq z \leq 1.4$, and measured the redshift number density and equivalent width distribution of Mg II absorbers with $W_{\mathrm{r}}(2796) \geq 0.02 \AA$.

Main results from this work include:

(1) The redshift path density of weak MgII absorbers was measured to be $d N / d z=1.74 \pm 0.11$. There is no evidence for evolution in the redshift path density of weak MgII absorbers, but the measured value of $\gamma=1.3 \pm 0.9$ is not constraining. Incorporating the MG1 sample of SS92 $\left[W_{\mathrm{r}}^{\min }(2796)=0.3 \AA\right]$, we find that the total number density per unit redshift for systems with $W_{\mathrm{r}}^{\min }(2796)=0.02 \AA$ at $\langle z\rangle=0.9$ is $d N / d z=2.65 \pm 0.15$. The $d N / d z$ of weak Mg II absorbers is roughly $5-7 \%$ of that of the Ly $\alpha$ forest with $W_{\mathrm{r}}(\operatorname{Ly} \alpha) \geq 0.1 \AA$ (Jannuzi et al. 1998). Thus, it is plausible that $\sim 5 \%$ of $z \sim 0.9$ "Ly $\alpha$ clouds" will have detectable Mg II absorption to $W_{\mathrm{r}}(2796)=0.02 \AA$.

(2) For $W_{\mathrm{r}}^{\min }(2796)=0.02 \AA$, MgII absorbers at $\langle z\rangle=0.9$ outnumber LLS absorbers by a factor of $3.8 \pm 1.1$. Given that the populations of strong Mg II absorbers and LLS are indistinguishable (Lanzetta 1988; SS92), this strongly suggests that virtually all of the weak MgII systems arise in sub-LLS environ- 
ments. It is possible that the weak Mg II systems have high metallicities, whether their ionization conditions are low or high (see Figures 10 and 11). Photoionization models, using Ferland's CLOUDY, are consistent with this conclusion; many weak MgII absorbers probably have $\left[Z / Z_{\odot}\right] \geq-1$. Lower metallicities require $N(\mathrm{HI})$ above the Lyman limit value, which is not allowed because the ratio of $d N / d z$ of weak Mg II absorbers to that of LLS absorbers requires that most all weak Mg II absorbers have $N\left(\mathrm{HI}_{\mathrm{I}}\right)$ near or below the Lyman limit value.

(3) The equivalent width distribution was found to follow a power law with slope $\delta \sim-1$ down to $W_{\mathrm{r}}(2796)=0.017 \AA$. There is no turnover or break in the equivalent width distribution for $W_{\mathrm{r}}(2706) \leq$ $0.3 \AA$ at $\langle z\rangle=0.9$. Previous measurements (TBSYK; LTW; SS92) of the power-law slope were likely biased to a steeper value due to the paucity of $W_{\mathrm{r}}(2796) \geq$ $1.3 \AA$ absorbers at redshifts $z<1$.4. A single powerlaw slope does not describe the equivalent width distribution over the full redshift range $0.3 \leq z \leq 2.2$. For $z<1.4$, there is a break (a significantly steeper slope) in the distribution for $W_{\mathrm{r}}(2796)>1.3 \AA$. The upper limit on this slope is $\delta=-2.3$. At $z \geq 2$, and perhaps as low as $z \sim 1.5$, it is expected that there would be a paucity of weak absorbers due to the increasing intensity of the UV extra-galactic background at higher redshifts.

(4) Most, if not all, of the individual clouds in these weak absorbers have $W_{\mathrm{r}}(2796) \leq 0.15 \AA$. Their line widths are narrow, with an average of $\sim 4 \mathrm{~km} \mathrm{~s}^{-1}$, implying temperatures of $\sim 25,000 \mathrm{~K}$. The presence of $\mathrm{MgI}$ is not correlated with $W_{\mathrm{r}}(2796)$ or with the Mg II doublet ratio. In fact, $\mathrm{Mg}$ I absorption is present in at least one component having $W_{\mathrm{r}}(2796)$ as small as $0.08 \AA$. Statistically, there appears to be no $W_{\mathrm{r}}(2796)$ threshold for the presence of $\mathrm{Mg}$ I down to $W_{\mathrm{r}}(2796) \sim$ $0.1 \AA$.

(5) The weak MgII absorbers may comprise a diverse population, including low, high, and multiphase ionization systems. The low ionization systems would be immediately recognizable by the presence of $W_{\mathrm{r}}(2600) / W_{\mathrm{r}}(2796) \geq 0.3$, though this criterion would be slightly relaxed if the gas was irongroup enhanced. In a single $\mathrm{Mg}$ II component with $b \leq 6 \mathrm{~km} \mathrm{~s}^{-1}$ and $W_{\mathrm{r}}(2796)<0.2 \AA$, the presence of CIV with $W_{\mathrm{r}}(1548) \geq 0.2 \AA$, and especially the simultaneous presence of FeII and CIV, may be due to multi-phase absorption or additional spatially distinct high ionization components in which $\mathrm{Mg}$ II has not been detected. In an absorber with $N_{\mathrm{c}}$ components in which the individual components have $b \leq 6 \mathrm{~km} \mathrm{~s}^{-1}$ and $W_{\mathrm{r}}(2796)<0.2 \AA$, multi-phase or additional high ionization components are likely if the total system CIV equivalent width is larger than $\sim N_{\mathrm{c}} \times 0.2 \AA$. This is especially true if the Mg II components have $W_{\mathrm{r}}(2600) / W_{\mathrm{r}}(2796) \geq 0.3$.

(6) The velocity clustering of weak MgII absorbers around strong ones [demarcated by $W_{\mathrm{r}}(2796)=0.3 \AA$ ] is consistent with a random distribution; a $\chi^{2}$ test yielded a probability of 0.18 that clustering was drawn from a random distribution. Furthermore, we examined the redshift clustering of the weak systems with respect to one another and found that the systems are also not inconsistent with a random distribution. The $\chi^{2}$ probability was 0.25 . We conclude that the QSO sight lines surveyed for this work are unbiased for the presence of weak MgII absorbers.

(7) We examined the range of absorber crosssections (physical sizes) required to account for the observed $d N / d z$ of all MgII absorbers. Assuming the radius-luminosity relationship between gas and galaxies and the cut off, $L_{\min }$, in the MgII absorbing galaxy luminosity function (Steidel 1995), we inferred an $R_{*}$ for $W_{\mathrm{r}}^{\min }(2796)=0.02 \AA$ Mg II absorbers of $63 h^{-1} \mathrm{kpc}$. We also explored the sensitivity of $R_{*}$ to the allowed ranges in the absorption radiusluminosity relationship and to $L_{\mathrm{min}}$, since it is not entirely clear that the relationships are applicable when the equivalent width detection threshold is lowered to $W_{\mathrm{r}}(2796)=0.02 \AA$. If there is no radius-luminosity dependence, then $R^{*}=53 h^{-1} \mathrm{kpc}$. If there is no luminosity cut off, then $R^{*}=46 h^{-1} \mathrm{kpc}$. We also explored the possibility that more than one population of luminous object may be associated with the absorbing gas by varying the normalization of the luminosity function. As suggested by Churchill \& Le Brun (1998), it is possible that low surface brightness (LSB) galaxies contribute to the measured $d N / d z$ of weak MgII absorbers. If LSB galaxies have the same absorbing properties as do the MgII selected galaxies observed by Steidel (1995), then the combined galaxy population would have $\Phi^{*} / \Phi_{\text {CFRS }}^{*} \sim 3$, where we have used the $\Phi^{*}$ measured by Dalcanton (1997, LSB galaxies) and by Steidel et al. (1994, MgII selected galaxies). A $\Phi^{*} / \Phi_{\text {CFRS }}^{*} \sim 3$ yields $R_{*} \sim 35 h^{-1} \mathrm{kpc}$. This is surprisingly consistent with the $R_{*}$ measured by Steidel (1995) for $W_{\mathrm{r}}(2796)>0.3 \AA$ absorption selected galaxies. It is not inconsistent with the data to suggest that a substantial fraction of the weak MgII 
absorbers are arising in LSB galaxies.

All this suggests that a great deal remains to be explored (and exploited) if we are to construct a comprehensive picture of chemical evolution and of the components of gas that, at least at one time, were associated with star forming environments. A central question now is if the galaxies selected by $\mathrm{Mg}$ II absorption with $W_{\mathrm{r}}(2796) \geq 0.3 \AA$ (Steidel 1995; Steidel et al. 1994) can tell the whole story or if they are the "tip" of the "iceberg". Are weak MgII absorbers analogous to the bulk of material that lies hidden below the "water line"? If so, it is not obvious that the surface brightnesses of the star forming environments associated with weak MgII absorbers are above the Freeman (1970) value. It could be that the successful broad-band imaging technique for identifying the luminous counterparts of strong $\mathrm{Mg}$ II absorbers in QSO fields will be ineffective for locating those associated with the weak MgII absorbers.

Conceivably, a "hidden" population, such as LSB galaxies, could significantly contribute to the weak MgII absorption cross section. Impey \& Bothun (1989), upon reexamining the selection effects and assumptions that go into the calculations of galaxy cross sections from QSO absorbers, found that LSB galaxies are expected to dominate the absorption cross section. So why is it that strong MgII absorbers are not associated with LSB galaxies more often than or as often as are normal high surface brightness galaxies? When strong MgII absorption is associated with an LSB galaxy, why is it that the absorbers are damped Ly $\alpha$ systems (Steidel et al. 1994; Le Brun et al. 1997)? And finally, why might weak absorbers preferentially select LSB galaxies? LSB galaxies typically have H I surface densities a factor of two lower than normal high surface brightness galaxies de Blok, McGaugh, $\&$ an der Hulst 1996) and lower metallicities (McGa igh 1994). Further, the inner $15 h^{-1} \mathrm{kpc}$ of LSB galaxies are found to have $N(\mathrm{HI})$ of a few $\times 10^{20} \mathrm{~cm}^{-2}$ (de Blok et al. 1996). It could be that the roughly half, or more, of the weak MgII absorbers are picking out those LSB galaxies that have metallicities on the high-end tail of the metallicity distribution at galactocentric distances of greater than $\sim 15 h^{-1} \mathrm{kpc}$, where the Hi environment is sub-Lyman limit and clouds cannot have large MgII column densities. In fact, at the low redshifts studied in this work, most sub-LLS absorbers are thought to be associated with LSB galaxies (Linder 1997; Salpeter 1993). Thus, it is possible that the strong MgII (and FeII) associated with damped Ly $\alpha$ absorbers in LSB galaxies arise in their inner regions, whereas only weak Mg II absorbing clouds can survive in their outer regions.

On the other hand, the absorption could arise in the outer disks and halos of high surface brightness galaxies (Le Brun et al. 1996; Lanzetta et al. 1995), or in the remnant material left over from the formation of galaxies and/or small galaxy groups (van Gorkom et. al. 1996; Le Brun et al. 1996; Bowen et al. 1995). For this scenario, it is expected that a kinematic spread of clouds would often be seen in the systems (Churchill et al. 1996), and that CIV might be present, either in a multi-phase environment or because the clouds are highly ionized Churchill 1998; Bergeron et al. 1994). Based upon observations, it seems less likely that the majority of weak MgII absorbers are arising in outer disks and halos of $L \geq 0.06 L^{*}$ galaxies [assuming the Freeman (1970) surface brightness]. This inference is not based upon statistical arguments, which require the sizes of the absorbing regions to be a plausible $63 h^{-1} \mathrm{kpc}$. It is based upon the reported lack of "interloper" galaxies seen within $\sim 20 \operatorname{arcsec}$ (a galactocentric distance of $\sim 90 h^{-1} \mathrm{kpc}$ at $z=0.9$ ) of the QSO in many of these fields (Steidel 1993, 1995). This further implies the idea of a "hidden" population of absorbers. However, we note that the kinematic models of Charlton \& Churchill (1998) may be suggesting that some fraction of the weak MgII absorbers do arise in the extended outer disks of galaxies to a projected galactocentric distance of $\sim 60 \mathrm{kpc}$. We are exploring techniques for detecting this putative population, with the hopes of exploiting it to greatly enhance our current understanding of how metals are distributed in the gaseous remnants of star formation.

This work has been supported in part by the National Science Foundation Grant AST-9617185, by NASA LTSA grant NAG5-6399, and by a Sigma-Xi Grant in Aid of Research issued to CWC while at the University of California, Santa Cruz, for travel to the telescope. CWC acknowledges support through the Eberly School of Science Distinguished Postdoctoral Fellowship at The Pennsylvania State University. JRR is partially supported as a Braddock Scholar in the Eberly College of Science at Penn State and through an NSF REU Supplement to AST-9529242. Special thanks are extended to Adam Dobrzycki for kindly working with us to search through the FOS/HST Archival spectra. Thanks to G. Ferland for making CLOUDY a public tool. We thank an anonymous referee for comments that led to an improved manuscript. This paper is dedicated in loving memory to Julius L. Nelson. 


\section{APPENDIX}

\section{Computation of the Redshift Path Density}

The number of sight lines in which a MgII doublet at redshift $z_{k}$ with rest-frame $\lambda 2796$ equivalent width greater than or equal to $W_{l}$ and with doublet ratio less than or equal to $\mathrm{DR}_{m}$ could be detected is given by

$$
g\left(W_{l}, z_{k}, \mathrm{DR}_{m}\right)=\sum_{i}^{N_{\mathrm{QSO}}} I_{\mathrm{db}}\left(z_{k}\right) I_{27}\left(W_{l}, z_{k}\right) I_{28}\left(W_{l}, z_{k}, \mathrm{DR}_{m}\right),
$$

where the sum is over all QSOs; $I_{\mathrm{db}}\left(z_{k}\right)=1$ if both transitions of the doublet at $z_{k}$ were captured by the CCD (could have been observed) and zero otherwise; $I_{27}=1$ if a $\lambda 2796$ transition could have been detected at the $5 \sigma$ significance level and zero otherwise; and, $I_{28}=1$ if the $\lambda 2803$ transition in a doublet with doublet ratio $\mathrm{DR}_{m}$ could have been detected at the $2.5 \sigma$ significance level and zero otherwise. The $I$ are the products of heavy-side step functions, $H[x]$, which have the binary dependence upon $x$ in that $H[x]=1$ if $x \geq 0$ and $H[x]=0$ if $x<0$. For QSO $i$, one searches the echelle orders beginning with $j=1$ in ascending order until the product $I_{\mathrm{db}} I_{27} I_{28}=1$ and then skips to the next QSO. We have dropped the $i, j$ subscripts from the $I$ and the $H$ functions for presentation purposes.

Each echelle order has an observed $\lambda^{\min }$ and $\lambda^{\max }$. Because there is duplicate coverage of the free spectral range for wavelengths below $5100 \AA$, the practical logistics of determining if a given Mg II doublet with $W_{l}(2796)$ and $\mathrm{DR}_{m}$ could have been detected is somewhat involved. Basically, there are three possibilities to test: (1) both transitions of the doublet could have been captured by the CCD on order $j$; (2) the $\lambda 2796$ transition could have been captured on order $j$ and the $\lambda 2803$ transition could have been captured on order $j+1$ (the doublet bridged the order gap); (3) both transitions of the doublet could have been captured on both order $j$ and order $j+1$ (this can occur only at the lowest redshifts). We refer to these as cases "1", "2", and "3" in the following discussion. Formally, the implementation of these three cases can be written as the logical "OR" of heavy-side step functions, $H_{\mathrm{db}}^{(1)}, H_{\mathrm{db}}^{(2)}$, and $H_{\mathrm{db}}^{(3)}$. We have

$$
I_{\mathrm{db}}\left(z_{k}\right)= \begin{cases}0 & \text { if } H_{\mathrm{db}}^{(1)}=H_{\mathrm{db}}^{(2)}=H_{\mathrm{db}}^{(3)}=0 \\ 1 & \text { otherwise }\end{cases}
$$

where the case "1" step function is given by,

$$
H_{\mathrm{db}}^{(1)}=H\left[\lambda_{k}^{(27)}-\lambda_{i, j}^{\min }\right] \cdot H\left[\lambda_{i, j}^{\max }-\lambda_{k}^{(28)}\right],
$$

where

$$
\begin{aligned}
& \lambda_{k}^{(27)}=2796.352\left(1+z_{k}\right), \\
& \lambda_{k}^{(28)}=2803.531\left(1+z_{k}\right),
\end{aligned}
$$

and $\lambda_{i, j}^{\min }$ and $\lambda_{i, j}^{\max }$ are the starting and ending observed wavelengths on order $j$ of QSO $i$. The case "2" and case "3" step functions are given by

$$
H_{\mathrm{db}}^{(2)}=H\left[\lambda_{k}^{(27)}-\lambda_{i, j}^{\min }\right] \cdot H\left[\lambda_{i, j}^{\max }-\lambda_{k}^{(27)}\right] \cdot H\left[\lambda_{k}^{(28)}-\lambda_{i, j+1}^{\min }\right] \cdot H\left[\lambda_{i, j+1}^{\max }-\lambda_{k}^{(28)}\right],
$$

and

$$
H_{\mathrm{db}}^{(3)}=H\left[\lambda_{k}^{(27)}-\lambda_{i, j}^{\min }\right] \cdot H\left[\lambda_{i, j}^{\max }-\lambda_{k}^{(28)}\right] \cdot H\left[\lambda_{k}^{(27)}-\lambda_{i, j+1}^{\min }\right] \cdot H\left[\lambda_{i, j+1}^{\max }-\lambda_{k}^{(28)}\right] .
$$

If $I_{\mathrm{db}}=0$ for a given $i, j$ pair, then a Mg II doublet at $z_{k}$, regardless of its rest-frame equivalent width or doublet ratio, could not have been observed along the QSO $i$ sight line. If the doublet could in principle be observed, then we evaluated $I_{27}$ and $I_{28}$. Again, we use a series of heavy-side step functions,

$$
I_{27}\left(W_{l}, z_{k}\right)= \begin{cases}0 & \text { if } H_{27}^{(1)}=H_{27}^{(2)}=H_{27}^{(3)}=0 \\ 1 & \text { otherwise }\end{cases}
$$


where the $H_{27}$ are given by

$$
H_{27}^{(1)}=H_{27}^{(2)}=H\left[W_{l}-W_{i, j}^{\min }\left(\lambda_{k}^{(27)}\right)\right] .
$$

and

$$
H_{27}^{(3)}=\max \left\{H\left[W_{l}-W_{i, j}^{\min }\left(\lambda_{k}^{(27)}\right)\right], H\left[W_{l}-W_{i, j+1}^{\min }\left(\lambda_{k}^{(27)}\right)\right]\right\} .
$$

If $I_{27}=1$ then a $\lambda 2796$ transition at $z_{k}$ with rest-frame equivalent width greater than or equal to $W_{l}$ is detectable to the $5 \sigma$ significance level. Testing for the detection of a MgII doublet with doublet ratio less than or equal to $\mathrm{DR}_{m}$ is written

$$
I_{28}\left(W_{l}, z_{k}, \mathrm{DR}_{m}\right)= \begin{cases}0 & \text { if } H_{28}^{(1)}=H_{28}^{(2)}=H_{28}^{(3)}=0 \\ 1 & \text { otherwise }\end{cases}
$$

where the $H_{28}$ are given by

$$
\begin{aligned}
& H_{28}^{(1)}=H\left[W_{l} / \mathrm{DR}_{m}-\frac{1}{2} W_{i, j}^{\min }\left(\lambda_{k}^{(28)}\right)\right] \\
& H_{28}^{(2)}=H\left[W_{l} / \mathrm{DR}_{m}-\frac{1}{2} W_{i, j+1}^{\min }\left(\lambda_{k}^{(28)}\right)\right]
\end{aligned}
$$

and, for completeness,

$$
H_{28}^{(3)}=\max \left\{H_{28}^{(1)}, H_{28}^{(2)}\right\}
$$

If $I_{28}=1$ then a Mg II $\lambda \lambda 2976,2803$ doublet at $z_{k}$ with rest-frame equivalent width greater than or equal to $W_{l}$ and with doublet ratio less than or equal to $\mathrm{DR}_{m}$ is detectable to our required significance level in QSO $i$. 


\section{REFERENCES}

Bahcall, J. N., et al. 1996, ApJ, 457, 19 (KP VII)

Bergeron, J. et al. 1994, ApJ, 436, 33

Bergeron, J., and Boissé, P. 1991, A\&A, 243, 344

Bergeron, J. and Stasińska, G. 1986, A\&A, 169, 1

Boissé, P., Bergeron, J., Le Brun, V., and Deharveng, J.-M. 1997, A\&A, accepted

Bowen, D. V., Blades, J. C., Pettini, M. 1995, ApJ, 448, 634

Caulet, A. 1989, ApJ, 340, 90

Charlton, J. C., and Churchill, C. W. 1996, ApJ, 465, 631

Charlton, J. C., and Churchill, C. W. 1998, ApJ, 499, in press

Churchill, C. W. 1995, Lick Observatory Technical Report, \#74

Churchill, C. W. 1997a, Ph. D. Thesis, University of California, Santa Cruz

Churchill, C. W. 1997b, in The Ultraviolet Universe at Low and High Redshift: Probing the Progress of Galaxy Evolution, eds. W. H. Waller, M. N. Fanelli, J. E. Hollis, \& A. C. Danks, (New York: AIP Press), 313

Churchill, C. W. 1998, in Structure and Evolution of the IGM from QSO Absorption Line Systems, Proceedings of the 13th IAP Colloquium, eds. P. Petitjean, and S. Charlot (Paris : Editions Frontieres), 229

Churchill, C. W., Charlton, J. C., Rigby, J. R., Januzzi, B. T., Kirhakos, S., and Vogt, S. S. 1998, in progress (Paper II)

Churchill, C. W., and Le Brun, V. 1998, ApJ, 499, in press

Churchill, C. W., Steidel, C. C., and Vogt, S. S, 1996, ApJ, 471,164

Churchill, C. W., Vogt, S. S., and Charlton, J. C. 1998, ApJS, to be submitted (CVC98)

Corbelli, E., Schneider, S. E., and Salpeter, E. E. 1989, AJ, 97, 390

Dalcanton, J. J., Spergel, D. N., Gunn, J. E., Smith, M., and Schneider, D. P. 1997, AJ, 114, 635

Dobrzycki, A., Bechtold, A., Wilden, B., Morita, M., Dobrzycka, D., Scott, J., Tran, K-V. 1998, ApJ, in preparation

de Blok, W. J. G., McGaugh, S. S., and van der Hulst, J. M. 1996, MNRAS, 283, 18

Ferland, G. 1996, Hazy, University of Kentucky Internal Report

Freeman, K. 1970, ApJ, 160, 811

Haardt, F., and Madau, P. 1996, ApJ, 461, 20

Holmberg, E. 1975, in Galaxies and the Universe, ed. A. Sandage, M. Sandage, \& J. Christian (Chicago: University of Chicago Press), 123

Impey, C., and Bothun, G. 1989, ApJ, 341, 89

Impey, C., Petry, C. E., Malkan, M. A., and Webb, W. 1996, ApJ, 463, 473

Jannuzi, B. T., et al., 1998, in press (KP XIII)

Kirhakos, S., et al. 1992, PASP 646, 1994

Kulkarni, V. P., and Fall, S. M. 1994, ApJ, 413, L63

Lanzetta, K. M., Turnshek, D. A., and Wolfe, A. M. 1987, ApJ, 322, 739 (LTW)

Lanzetta, K. M. 1988, ApJ, 332, 96

Lanzetta. K. M., Bowen, D. B., Tytler, D., and Webb, J. K. 1995, ApJ, 442, 538

Lauroesch, J. T., Truran, J. W., Welty, D. E., and York, D. G. 1996, PASP, 108, 641

Le Brun, V., Bergeron, J., Boissé, P., and Deharving, J. M. 1997, A\&A, 321, 733

Le Brun, V., Bergeron, J., Boissé, P. 1996, A\&A, 306, 691
Lilly, S. J., Tresse, L., Hammer, F., Crampton, D., and Le Fèvre, O. 1995, 455, 108

Linder, S. M. 1997, ApJ, in press

McGaugh, S. 1994, ApJ, 426, 135

Petitjean, P., and Bergeron, J. 1990, A\&A, 231, 309 (PB90)

Petitjean, P., Rauch, M., and Carswell, R. F. 1994, A\&A, 291, 29

Salpeter, E. E. 1993, AJ, 106, 1265

Sargent, W. L. W., Boksenberg, A., and Steidel, C. C. 1988, ApJS, 68, 539

Sargent, W. L. W., Steidel, C. C., and Boksenberg, A. 1988, ApJ, 334, 22 (SSB)

Savage, B. D., and Sembach, K. R. 1996, ARAA, 34, 279

Savage, B. D., and Sembach, K. R. 1991, ApJ, 379, 245

Schechter, P. 1976, ApJ, 203, 297

Schneider, D. P. et al. 1993, ApJS, 87, 45

Sembach, K. R., and Savage, B. D. 1992, ApJS, 83, 147

Steidel, C. C. 1993, in The Environment and Evolution of Galaxies, eds. J. M. Shull \& H. A. Thronson, Jr., (Dordrecht : Kluwer Academic Publishers), 263

Steidel, C. C., Pettini, M., Dickinson, M., and Persson, S. E. 1994, AJ, 108, 2046

Steidel, C. C. 1995, in ESO Workshop on Quasar Absorption Lines, ed. G. Meylan, (Garching : Springer-Verlag), 139

Steidel, C. C. 1996, private communication

Steidel, C. C., Dickinson, M., and Persson, S. E. 1994, ApJ, L75

Steidel, C. C., and Sargent, W. L. W. 1992, ApJS, 80, 1 (SS92)

Stengler-Larrea, E. A., et al. 1995, ApJ, 444, 64

Thimm, G. 1995, in ESO Workshop on Quasar Absorption Lines, ed. G. Meylan, (Garching : Springer-Verlag), 169

Tripp, T. M., Lu, L., and Savage, B. D. 1997, ApJS, 112, 1

Tytler, D., Boksenberg, A., Sargent, W. L. W., Young, P., and Kunth, D. 1987, ApJS, 64, 667 (TBSYK)

Uomoto, A. 1984, ApJ, 284, 497

van Gorkom, J. H., Bahcall, J. N., Jannuzi, B. T., and Schneider, D. P. 1993, AJ, 106, 2213

van Gorkom, J. H., Carilli, C. L., Stocke, J. T., Perlman, E. S., Shull, J. M. 1996, AJ, 112, 1397

Vogt, S. S., et al. 1994, SPIE, 2198, 362

Womble, D. 1995 Workshop on Quasar Absorption Lines, ed. G. Meylan, (Garching : Springer-Verlag), 158

Yanny, B., and York, D. G. 1992, ApJ, 391, 569

This 2-column preprint was prepared with the AAS LATEX macros v4.0. 
TABLE 1

JoURNAL OF HIRES/KECK OBSERVATIONS

\begin{tabular}{cccrc}
\hline \hline Object & $z_{\mathrm{em}}$ & Date $[\mathrm{UT}]$ & ${\text { Exp }[\mathrm{s}]^{\mathrm{a}}}$ & $\lambda$ Range $[\AA]^{\mathrm{b}}$ \\
\hline $0002+051$ & 1.899 & 1994 Jul 05 & 2700 & $3655.7-6079.0$ \\
$0058+019$ & 1.959 & 1996 Jul 18 & 3000 & $3766.2-5791.3$ \\
$0117+212$ & 1.491 & 1995 Jan 23 & 5400 & $4317.7-6775.1$ \\
$0420-014$ & 0.915 & 1995 Jan 23 & 3600 & $3810.5-6304.9$ \\
$0450-132$ & 2.253 & 1995 Jan 24 & 5400 & $3986.5-6424.5$ \\
$0454+039$ & 1.343 & 1995 Jan 22 & 4500 & $3765.8-6198.9$ \\
$0454-220$ & 0.534 & 1995 Jan 22 & 5400 & $3765.8-6198.9$ \\
$0823-223$ & $\cdots$ & 1995 Jan 24 & 3600 & $3977.8-6411.8$ \\
$0958+551$ & 1.755 & 1995 Jan 23 & 3600 & $5400.0-7830.0$ \\
$1148+384$ & 1.299 & 1995 Jan 24 & 5400 & $3986.5-6424.5$ \\
$1206+459$ & 1.155 & 1995 Jan 23 & 3600 & $3810.5-6304.9$ \\
$1213-003$ & 2.691 & 1995 Jan 24 & 5200 & $5008.1-7356.7$ \\
$1222+228$ & 2.040 & 1995 Jan 23 & 3600 & $3810.5-6304.9$ \\
$1225+317$ & 2.219 & 1995 Jan 24 & 2400 & $5737.5-8194.7$ \\
$1241+174$ & 1.282 & 1995 Jan 22 & 2400 & $3765.8-6189.9$ \\
$1248+401$ & 1.032 & 1995 Jan 22 & 4200 & $3765.8-6189.9$ \\
$1254+044$ & 1.018 & 1995 Jan 22 & 2400 & $3765.8-6189.9$ \\
$1317+274$ & 1.014 & 1995 Jan 23 & 3600 & $3810.5-6304.9$ \\
$1329+412$ & 1.937 & 1996 Jul 18 & 6300 & $3766.2-5791.3$ \\
$1354+193$ & 0.719 & 1995 Jan 22 & 3600 & $3765.8-6189.9$ \\
$1421+331$ & 1.906 & 1995 Jan 23 & 3600 & $3818.6-6316.9$ \\
$1548+092$ & 2.749 & 1996 Jul 19 & 3600 & $3766.2-5791.3$ \\
$1622+235$ & 0.927 & 1994 Jul 04 & 16200 & $3726.9-6191.0$ \\
& & 1994 Jul 05 & 3040 & \\
$1634+704$ & 1.335 & 1994 Jul 04 & 2700 & $3723.3-6185.7$ \\
& & 1994 Jul 05 & 5400 & \\
$2128-123$ & 0.501 & 1996 Jul 19 & 3900 & $3766.2-5791.3$ \\
$2145+064$ & 0.999 & 1996 Jul 18 & 4500 & $3766.2-5791.3$ \\
\hline
\end{tabular}

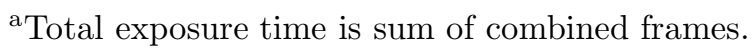

${ }^{\mathrm{b}}$ Above $5100 \AA$ there are small gaps in the wavelength coverage. 
TABLE 2

SAMPle of Absorbers

\begin{tabular}{|c|c|c|c|c|c|c|}
\hline ID & $z_{\text {abs }}$ & QSO & $\begin{array}{c}\omega_{v} \\
{\left[\mathrm{~km} \mathrm{~s}^{-1}\right]}\end{array}$ & $\begin{array}{c}\mathrm{W}_{\mathrm{r}}(\lambda 2796) \\
{[\AA]}\end{array}$ & DR & $Z\left(W_{\mathrm{r}}, \mathrm{DR}\right)$ \\
\hline S1 & 0.456415 & $1421+331$ & $6.45 \pm 0.98$ & $0.179 \pm 0.019$ & $1.15 \pm 0.09$ & 17.24 \\
\hline S2 & 0.500786 & $1329+412$ & $25.57 \pm 1.39$ & $0.258 \pm 0.035$ & $1.33 \pm 0.41$ & 17.26 \\
\hline $\mathrm{S} 3^{\mathrm{a}}$ & 0.521498 & $1354+193$ & $6.57 \pm 1.14$ & $0.030 \pm 0.007$ & $1.31 \pm 0.68$ & 15.74 \\
\hline S4 & 0.550202 & $1222+228$ & $9.58 \pm 1.25$ & $0.080 \pm 0.014$ & $1.30 \pm 0.35$ & 17.05 \\
\hline S5 & 0.558443 & $1241+174$ & $17.17 \pm 1.10$ & $0.135 \pm 0.014$ & $2.03 \pm 0.62$ & 17.19 \\
\hline S6 & 0.591485 & $0002+051$ & $4.96 \pm 0.73$ & $0.103 \pm 0.008$ & $1.62 \pm 0.31$ & 17.13 \\
\hline S7 & 0.642827 & $0454+039$ & $4.77 \pm 0.57$ & $0.118 \pm 0.008$ & $1.46 \pm 0.19$ & 17.18 \\
\hline $\mathrm{S} 8^{\mathrm{a}}$ & 0.705472 & $0823-223$ & $11.86 \pm 0.68$ & $0.092 \pm 0.007$ & $2.09 \pm 0.70$ & 17.06 \\
\hline S9 & 0.725175 & $0058+019$ & $9.30 \pm 0.45$ & $0.253 \pm 0.012$ & $1.26 \pm 0.12$ & 17.26 \\
\hline S10 & 0.729071 & $0117+212$ & $47.4 \pm 1.39$ & $0.238 \pm 0.009$ & $1.71 \pm 0.18$ & 17.25 \\
\hline S11 & 0.770646 & $1548+092$ & $9.53 \pm 0.85$ & $0.234 \pm 0.024$ & $1.19 \pm 0.19$ & 17.25 \\
\hline S12 & 0.818157 & $1634+706$ & $3.14 \pm 1.56$ & $0.030 \pm 0.018$ & $1.68 \pm 0.61$ & 17.67 \\
\hline S13 & 0.843247 & $1421+331$ & $3.08 \pm 0.75$ & $0.086 \pm 0.008$ & $1.01 \pm 0.13$ & 15.09 \\
\hline S14 & 0.854554 & $1248+401$ & $62.68 \pm 9.42$ & $0.235 \pm 0.014$ & $1.76 \pm 0.25$ & 17.25 \\
\hline $\mathrm{S} 15^{\mathrm{a}}$ & 0.866529 & $0002+051$ & $1.68 \pm 0.54$ & $0.023 \pm 0.008$ & $1.62 \pm 0.35$ & 14.44 \\
\hline $\mathrm{S} 16^{\mathrm{a}}$ & 0.895493 & $1241+174$ & $4.95 \pm 1.21$ & $0.018 \pm 0.005$ & $1.87 \pm 0.41$ & 12.71 \\
\hline S17 & 0.905554 & $1634+706$ & $4.07 \pm 0.64$ & $0.064 \pm 0.004$ & $1.42 \pm 0.17$ & 16.96 \\
\hline $\mathrm{S} 18^{\mathrm{a}}$ & 0.931502 & $0454+039$ & $4.36 \pm 0.89$ & $0.042 \pm 0.005$ & $1.84 \pm 0.28$ & 16.34 \\
\hline S19 & 0.934283 & $1206+459$ & $7.67 \pm 0.83$ & $0.049 \pm 0.005$ & $2.02 \pm 0.50$ & 16.53 \\
\hline S20 & 0.956029 & $0002+051$ & $6.24 \pm 0.98$ & $0.052 \pm 0.007$ & $2.07 \pm 0.61$ & 16.60 \\
\hline S21 & 0.973867 & $1329+412$ & $26.79 \pm 2.72$ & $0.181 \pm 0.035$ & $1.41 \pm 0.62$ & 17.24 \\
\hline S22 & 0.998358 & $1329+412$ & $6.72 \pm 0.65$ & $0.142 \pm 0.010$ & $1.51 \pm 0.26$ & 17.22 \\
\hline $\mathrm{S} 23$ & 1.041443 & $1634+706$ & $17.19 \pm 1.07$ & $0.097 \pm 0.008$ & $2.10 \pm 0.44$ & 17.08 \\
\hline S24 & 1.127698 & $1213-003$ & $4.17 \pm 1.07$ & $0.036 \pm 0.006$ & $1.86 \pm 0.69$ & 16.03 \\
\hline $\mathrm{S} 25$ & 1.211316 & $0958+551$ & $3.43 \pm 1.08$ & $0.060 \pm 0.007$ & $1.36 \pm 0.34$ & 16.93 \\
\hline S26 & 1.229475 & $0450-132$ & $10.00 \pm 0.93$ & $0.135 \pm 0.010$ & $1.29 \pm 0.16$ & 17.21 \\
\hline S27 & 1.232440 & $0450-132$ & $22.72 \pm 2.45$ & $0.101 \pm 0.009$ & $1.44 \pm 0.21$ & 17.13 \\
\hline $\mathrm{S} 28$ & 1.272377 & $0958+551$ & $4.86 \pm 0.62$ & $0.081 \pm 0.007$ & $1.45 \pm 0.24$ & 17.04 \\
\hline S29 & 1.325004 & $0117+212$ & $76.09 \pm 1.46$ & $0.291 \pm 0.011$ & $1.60 \pm 0.11$ & 17.26 \\
\hline $\mathrm{S} 30^{\mathrm{b}}$ & 1.342969 & $0117+212$ & $44.95 \pm 3.67$ & $0.153 \pm 0.008$ & $\ldots$ & $\ldots$ \\
\hline
\end{tabular}

${ }^{a}$ Quantites obtained using Gaussian fitting to data.

${ }^{\mathrm{b}}$ The $\lambda 2796$ transition was not covered. All quantities based upon $\lambda 2803$ transition. 
TABLE 3

System Properties

\begin{tabular}{|c|c|c|}
\hline Ion/Tran & $\begin{array}{l}\lambda_{\mathrm{abss}^{\mathrm{a}}} \\
{[\AA \bar{\AA}]}\end{array}$ & $\begin{array}{c}\mathrm{EW}_{\mathrm{r}} \\
{[\AA]}\end{array}$ \\
\hline S1 & $\mathrm{Q} 1421+331$ & $z_{\mathrm{abs}}=0.45642$ \\
\hline Mg II 2796 & 4072.649 & $0.179 \pm 0.019$ \\
\hline Mg II 2803 & 4083.105 & $0.155 \pm 0.020$ \\
\hline Mg I 2853 & 4155.100 & $<0.014$ \\
\hline $\mathrm{S} 2$ & $\mathrm{Q} 1329+412$ & $z_{\mathrm{abs}}=0.50079$ \\
\hline FeII 2587 & 3882.008 & $<0.092$ \\
\hline FeII 2600 & 3902.303 & $<0.100$ \\
\hline Mg II 2796 & 4196.726 & $0.258 \pm 0.035$ \\
\hline Mg II 2803 & 4207.500 & $0.194 \pm 0.054$ \\
\hline $\operatorname{Mg}$ I 2853 & 4281.688 & $<0.038$ \\
\hline S3 & $\mathrm{Q} 1354+193$ & $z_{\mathrm{abs}}=0.52149$ \\
\hline FeII 2587 & 3935.583 & $<0.016$ \\
\hline FeII 2600 & 3956.158 & $<0.012$ \\
\hline Mg II $2796^{b}$ & 4254.644 & $0.030 \pm 0.007$ \\
\hline Mg II 2803 & 4265.567 & $0.023 \pm 0.010$ \\
\hline Mg I 2853 & 4340.779 & $<0.007$ \\
\hline $\mathrm{S} 4$ & $\mathrm{Q} 1222+228$ & $z_{\mathrm{abs}}=0.55020$ \\
\hline FeII 2587 & 4009.830 & $<0.011$ \\
\hline FeII 2600 & 4030.793 & $<0.009$ \\
\hline Mg II 2796 & 4334.910 & $0.080 \pm 0.014$ \\
\hline Mg II 2803 & 4346.039 & $0.061 \pm 0.013$ \\
\hline $\operatorname{Mg}$ I 2853 & 4422.670 & $<0.008$ \\
\hline S5 & $\mathrm{Q} 1241+174$ & $z_{\mathrm{abs}}=0.55844$ \\
\hline FeII $2587^{\mathrm{c}}$ & 4031.147 & $\ldots$ \\
\hline FeII 2600 & 4052.221 & $<0.012$ \\
\hline Mg II 2796 & 4357.955 & $0.135 \pm 0.014$ \\
\hline Mg II 2803 & 4369.143 & $0.066 \pm 0.019$ \\
\hline Mg I 2853 & 4446.182 & $<0.008$ \\
\hline
\end{tabular}


TABLE 3-Continued

\begin{tabular}{|c|c|c|}
\hline Ion/Tran & $\begin{array}{c}\lambda_{\mathrm{abs}^{\mathrm{a}}} \\
{[\AA \AA]}\end{array}$ & $\begin{array}{c}\mathrm{EW}_{\mathrm{r}} \\
{[\AA]}\end{array}$ \\
\hline $\mathrm{S} 6$ & $\mathrm{Q} 0002+051$ & $z_{\mathrm{abs}}=0.59149$ \\
\hline FeII 2344 & 3730.781 & $<0.026$ \\
\hline FeII 2374 & 3778.919 & $<0.032$ \\
\hline FeII 2383 & 3792.135 & $<0.020$ \\
\hline FeII 2587 & 4116.615 & $<0.014$ \\
\hline FeII 2600 & 4138.136 & $<0.012$ \\
\hline Mg II 2796 & 4450.352 & $0.103 \pm 0.008$ \\
\hline Mg II 2803 & 4461.778 & $0.064 \pm 0.011$ \\
\hline Mg I 2853 & 4540.449 & $<0.010$ \\
\hline S7 & $\mathrm{Q} 0454+039$ & $z_{\mathrm{abs}}=0.64283$ \\
\hline FeII 2344 & 3851.138 & $<0.031$ \\
\hline FeII 2374 & 3900.829 & $<0.019$ \\
\hline FeII 2383 & 3914.471 & $0.029 \pm 0.005$ \\
\hline FeII $2587^{\mathrm{d}}$ & 4249.418 & $0.014 \pm 0.004$ \\
\hline FeII 2600 & 4271.634 & $0.037 \pm 0.014$ \\
\hline Mg II 2796 & 4593.923 & $0.118 \pm 0.008$ \\
\hline Mg II 2803 & 4605.716 & $0.081 \pm 0.009$ \\
\hline $\operatorname{Mg}_{\text {I }} 2853$ & 4686.926 & $<0.005$ \\
\hline S8 & Q0823 - 223 & $z_{\mathrm{abs}}=0.70547$ \\
\hline FeII 2344 & 3997.991 & $<0.019$ \\
\hline FeII 2374 & 4049.577 & $<0.016$ \\
\hline FeII 2383 & 4063.739 & $<0.017$ \\
\hline FeII 2587 & 4411.459 & $<0.008$ \\
\hline FeII 2600 & 4434.522 & $<0.008$ \\
\hline Mg II 2796 & 4769.100 & $0.092 \pm 0.007$ \\
\hline Mg II 2803 & 4781.344 & $0.044 \pm 0.011$ \\
\hline Mg I 2853 & 4865.650 & $<0.005$ \\
\hline S9 & Q0058+019 & $z_{\mathrm{abs}}=0.72518$ \\
\hline FeII 2344 & 4044.179 & $<0.026$ \\
\hline FeII 2374 & 4096.361 & $<0.020$ \\
\hline FeII 2383 & 4110.687 & $<0.018$ \\
\hline FeII 2587 & 4462.424 & $<0.013$ \\
\hline FeII $2600^{\mathrm{d}}$ & 4485.753 & $0.017 \pm 0.005$ \\
\hline Mg II 2796 & 4824.197 & $0.253 \pm 0.012$ \\
\hline Mg II 2803 & 4836.582 & $0.201 \pm 0.016$ \\
\hline $\operatorname{Mg}$ I 2853 & 4921.862 & $0.041 \pm 0.026$ \\
\hline
\end{tabular}


TABLE 3-Continued

\begin{tabular}{|c|c|c|}
\hline Ion/Tran & $\begin{array}{c}\lambda_{\mathrm{abs}^{\mathrm{a}}} \\
{[\AA \AA]}\end{array}$ & $\begin{array}{c}\mathrm{EW}_{\mathrm{r}} \\
{[\AA]}\end{array}$ \\
\hline S10 & $\mathrm{Q} 0117+212$ & $z_{\mathrm{abs}}=0.72907$ \\
\hline FeII $2587^{d}$ & 4472.502 & $0.012 \pm 0.002$ \\
\hline FeII 2600 & 4495.884 & $0.075 \pm 0.016$ \\
\hline Mg II 2796 & 4835.091 & $0.238 \pm 0.009$ \\
\hline Mg II 2803 & 4847.504 & $0.132 \pm 0.013$ \\
\hline Mg I 2853 & 4932.977 & $0.013 \pm 0.009$ \\
\hline S11 & $\mathrm{Q} 1548+093$ & $z_{\mathrm{abs}}=0.77065$ \\
\hline FeII 2374 & 4204.330 & $<0.190$ \\
\hline FeII 2383 & 4219.033 & $<0.131$ \\
\hline FeII $2587^{\mathrm{d}}$ & 4580.041 & $0.143 \pm 0.006$ \\
\hline FeII 2600 & 4603.986 & $0.101 \pm 0.027$ \\
\hline Mg II 2796 & 4951.349 & $0.234 \pm 0.024$ \\
\hline Mg II 2803 & 4964.061 & $0.197 \pm 0.025$ \\
\hline Mg I 2853 & 5051.589 & $0.051 \pm 0.059$ \\
\hline S12 & $\mathrm{Q} 1634+706$ & $z_{\mathrm{abs}}=0.81816$ \\
\hline FeII 2344 & 4262.149 & $<0.019$ \\
\hline FeII 2374 & 4317.143 & $<0.017$ \\
\hline FeII 2383 & 4332.241 & $<0.019$ \\
\hline FeII 2587 & 4702.936 & $<0.007$ \\
\hline FeII 2600 & 4727.523 & $<0.008$ \\
\hline Mg II 2796 & 5084.207 & $0.030 \pm 0.005$ \\
\hline Mg II 2803 & 5097.260 & $0.018 \pm 0.006$ \\
\hline $\operatorname{Mg}$ I 2853 & 5187.136 & $<0.004$ \\
\hline S13 & $\mathrm{Q} 1421+331$ & $z_{\mathrm{abs}}=0.84325$ \\
\hline FeII 2344 & 4320.965 & $0.041 \pm 0.015$ \\
\hline FeII 2374 & 4376.718 & $0.028 \pm 0.018$ \\
\hline FeII 2383 & 4392.024 & $0.068 \pm 0.011$ \\
\hline FeII 2587 & 4767.835 & $0.048 \pm 0.009$ \\
\hline FeII 2600 & 4792.761 & $0.071 \pm 0.014$ \\
\hline Mg II 2796 & 5154.367 & $0.086 \pm 0.008$ \\
\hline Mg II 2803 & 5167.600 & $0.085 \pm 0.007$ \\
\hline Mg I 2853 & 5258.717 & $0.022 \pm 0.009$ \\
\hline
\end{tabular}


TABle 3-Continued

\begin{tabular}{|c|c|c|}
\hline Ion/Tran & $\begin{array}{c}\lambda_{\mathrm{abs}}{ }^{\mathrm{a}} \\
{[\AA \AA]}\end{array}$ & $\begin{array}{c}\mathrm{EW}_{\mathrm{r}} \\
{[\AA]}\end{array}$ \\
\hline S14 & $\mathrm{Q} 1248+401$ & $z_{\mathrm{abs}}=0.85455$ \\
\hline FeII 2344 & 4347.471 & $0.024 \pm 0.010$ \\
\hline FeII 2374 & 4403.567 & $<0.023$ \\
\hline FeII 2383 & 4418.966 & $0.035 \pm 0.010$ \\
\hline FeII 2587 & 4797.082 & $0.010 \pm 0.009$ \\
\hline FeII 2600 & 4822.161 & $0.031 \pm 0.007$ \\
\hline Mg II 2796 & 5185.986 & $0.253 \pm 0.014$ \\
\hline Mg II 2803 & 5199.300 & $0.136 \pm 0.017$ \\
\hline Mg I 2853 & 5290.976 & $<0.019$ \\
\hline S15 & $\mathrm{Q} 0002+051$ & $z_{\mathrm{abs}}=0.86653$ \\
\hline FeII 2344 & 4375.543 & $<0.004$ \\
\hline FeII 2374 & 4432.001 & $<0.009$ \\
\hline FeII 2383 & 4447.500 & $<0.006$ \\
\hline FeII 2587 & 4828.057 & $<0.007$ \\
\hline FeII 2600 & 4853.298 & $<0.010$ \\
\hline Mg II 2796 & 5219.472 & $0.023 \pm 0.008$ \\
\hline Mg II 2803 & 5232.872 & $0.014 \pm 0.007$ \\
\hline Mg I 2853 & 5325.140 & $<0.007$ \\
\hline S16 & $\mathrm{Q} 1241+174$ & $z_{\mathrm{abs}}=0.89549$ \\
\hline FeII 2344 & 4443.441 & $<0.006$ \\
\hline FeII 2374 & 4500.775 & $<0.006$ \\
\hline FeII 2383 & 4516.514 & $<0.005$ \\
\hline FeII 2587 & 4902.977 & $<0.005$ \\
\hline FeII 2600 & 4928.610 & $<0.005$ \\
\hline Mg II 2796 & 5300.466 & $0.018 \pm 0.005$ \\
\hline Mg II 2803 & 5314.073 & $0.010 \pm 0.005$ \\
\hline Mg I 2853 & 5407.773 & $<0.004$ \\
\hline S17 & $\mathrm{Q} 1634+706$ & $z_{\mathrm{abs}}=0.90555$ \\
\hline FeII 2344 & 4467.026 & $<0.008$ \\
\hline FeII 2374 & 4524.664 & $<0.008$ \\
\hline FeII 2383 & 4540.487 & $<0.010$ \\
\hline FeII 2587 & 4929.001 & $<0.005$ \\
\hline FeII 2600 & 4954.770 & $<0.005$ \\
\hline Mg II 2796 & 5328.600 & $0.064 \pm 0.004$ \\
\hline Mg II 2803 & 5342.280 & $0.045 \pm 0.005$ \\
\hline Mg I 2853 & 5436.477 & $<0.004$ \\
\hline
\end{tabular}


TABLE 3-Continued

\begin{tabular}{|c|c|c|}
\hline Ion/Tran & $\begin{array}{c}\lambda_{\mathrm{abs}^{\mathrm{a}}} \\
{[\AA]}\end{array}$ & $\begin{array}{c}\mathrm{EW}_{\mathrm{r}} \\
{[\AA]}\end{array}$ \\
\hline S18 & $\mathrm{Q} 0454+039$ & $z_{\mathrm{abs}}=0.93150$ \\
\hline FeII 2344 & 4527.854 & $<0.005$ \\
\hline FeII 2374 & 4586.277 & $<0.004$ \\
\hline FeII 2383 & 4602.315 & $0.030 \pm 0.008$ \\
\hline FeII 2587 & 4996.120 & $<0.004$ \\
\hline FeII $2600^{\mathrm{e}}$ & 5022.239 & $\ldots$ \\
\hline MgII 2796 & 5401.159 & $0.043 \pm 0.005$ \\
\hline Mg II 2803 & 5415.026 & $0.023 \pm 0.005$ \\
\hline Mg I 2853 & 5510.506 & $<0.005$ \\
\hline S19 & $\mathrm{Q} 1206+459$ & $z_{\mathrm{abs}}=0.93428$ \\
\hline FeII 2344 & 4534.373 & $<0.005$ \\
\hline FeII $2374^{\mathrm{c}}$ & 4592.880 & $\ldots$ \\
\hline FeII 2383 & 4608.942 & $<0.005$ \\
\hline FeII 2587 & 5003.313 & $<0.004$ \\
\hline FeII 2600 & 5029.470 & $<0.004$ \\
\hline MgII 2796 & 5408.936 & $0.049 \pm 0.005$ \\
\hline Mg II 2803 & 5422.822 & $0.024 \pm 0.005$ \\
\hline Mg I 2853 & 5518.440 & $<0.004$ \\
\hline S20 & $\mathrm{Q} 0002+051$ & $z_{\mathrm{abs}}=0.95603$ \\
\hline FeII 2344 & 4585.351 & $<0.005$ \\
\hline FeII 2374 & 4644.515 & $<0.007$ \\
\hline FeII 2383 & 4660.757 & $<0.009$ \\
\hline FeII 2587 & 5059.562 & $<0.008$ \\
\hline FeII 2600 & 5086.014 & $<0.005$ \\
\hline Mg II 2796 & 5469.746 & $0.052 \pm 0.007$ \\
\hline Mg II 2803 & 5483.788 & $0.025 \pm 0.007$ \\
\hline MgI 2853 & 5580.480 & $<0.005$ \\
\hline $\mathrm{S} 21$ & $\mathrm{Q} 1329+412$ & $z_{\mathrm{abs}}=0.97387$ \\
\hline FeII 2344 & 4627.167 & $<0.025$ \\
\hline FeII 2374 & 4686.871 & $<0.032$ \\
\hline FeII 2383 & 4703.261 & $<0.027$ \\
\hline FeII 2587 & 5105.703 & $<0.026$ \\
\hline FeII 2600 & 5132.395 & $<0.028$ \\
\hline Mg II 2796 & 5519.627 & $0.181 \pm 0.035$ \\
\hline Mg II 2803 & 5533.797 & $0.128 \pm 0.050$ \\
\hline Mg I 2853 & 5631.371 & $<0.024$ \\
\hline
\end{tabular}


TABLE 3-Continued

\begin{tabular}{|c|c|c|}
\hline Ion/Tran & $\begin{array}{c}\lambda_{\mathrm{abss}^{\mathrm{a}}} \\
{[\AA \AA]}\end{array}$ & $\begin{array}{c}\mathrm{EW}_{\mathrm{r}} \\
{[\AA]}\end{array}$ \\
\hline S22 & $\mathrm{Q} 1329+412$ & $z_{\mathrm{abs}}=0.99836$ \\
\hline FeII 2344 & 4684.579 & $0.044 \pm 0.016$ \\
\hline FeII 2374 & 4745.024 & $<0.011$ \\
\hline FeII 2383 & 4761.617 & $0.061 \pm 0.013$ \\
\hline FeII 2587 & 5169.053 & $<0.011$ \\
\hline FeII 2600 & 5196.076 & $0.058 \pm 0.017$ \\
\hline Mg II 2796 & 5588.112 & $0.142 \pm 0.010$ \\
\hline Mg II 2803 & 5602.459 & $0.094 \pm 0.015$ \\
\hline $\mathrm{S} 23$ & $\mathrm{Q} 1634+706$ & $z_{\mathrm{abs}}=1.04144$ \\
\hline FeII 2344 & 4785.579 & $<0.008$ \\
\hline FeII 2374 & 4847.327 & $<0.006$ \\
\hline FeII 2383 & 4864.279 & $<0.005$ \\
\hline FeII 2600 & 5308.105 & $<0.038$ \\
\hline Mg II 2796 & 5708.593 & $0.097 \pm 0.008$ \\
\hline Mg II 2803 & 5723.249 & $0.046 \pm 0.009$ \\
\hline Mg I 2853 & 5824.163 & $<0.003$ \\
\hline $\mathrm{S} 24$ & $\mathrm{Q} 1213-003$ & $z_{\mathrm{abs}}=1.12770$ \\
\hline FeII 2374 & 5052.136 & $<0.007$ \\
\hline FeII 2383 & 5069.804 & $<0.014$ \\
\hline FeII 2587 & 5503.610 & $<0.008$ \\
\hline FeII 2600 & 5532.383 & $<0.010$ \\
\hline Mg II 2796 & 5949.793 & $0.036 \pm 0.006$ \\
\hline Mg II 2803 & 5965.067 & $0.019 \pm 0.006$ \\
\hline Mg I 2853 & 6070.246 & $<0.005$ \\
\hline $\mathrm{S} 25$ & Q0958+551 & $z_{\mathrm{abs}}=1.21132$ \\
\hline FeII 2587 & 5719.901 & $<0.011$ \\
\hline FeII 2600 & 5749.804 & $<0.006$ \\
\hline Mg II 2796 & 6183.618 & $0.060 \pm 0.007$ \\
\hline Mg II 2803 & 6199.493 & $0.044 \pm 0.010$ \\
\hline $\operatorname{Mg}$ I 2853 & 6308.805 & $<0.010$ \\
\hline
\end{tabular}


TABLE 3-Continued

\begin{tabular}{|c|c|c|}
\hline Ion/Tran & $\begin{array}{c}\lambda_{\mathrm{abss}^{\mathrm{a}}} \\
{[\AA]}\end{array}$ & $\begin{array}{c}\mathrm{EW}_{\mathrm{r}} \\
{[\AA]}\end{array}$ \\
\hline S26 & Q0450 - 132 & $z_{\mathrm{abs}}=1.22948$ \\
\hline AliII 1855 & 4135.017 & $0.042 \pm 0.009$ \\
\hline AlıII 1863 & 4153.092 & $0.010 \pm 0.005$ \\
\hline FeII 2344 & 5226.367 & $0.015 \pm 0.014$ \\
\hline FeII 2383 & 5312.315 & $0.037 \pm 0.010$ \\
\hline FeII 2587 & 5766.872 & $<0.007$ \\
\hline FeII 2600 & 5797.020 & $0.039 \pm 0.017$ \\
\hline Mg II 2796 & 6234.397 & $0.135 \pm 0.010$ \\
\hline Mg II 2803 & 6250.402 & $0.105 \pm 0.010$ \\
\hline $\operatorname{Mg}$ I 2853 & 6360.612 & $<0.007$ \\
\hline $\mathrm{S} 27$ & Q0450 - 132 & $z_{\mathrm{abs}}=1.23244$ \\
\hline FeII 2344 & 5233.317 & $<0.008$ \\
\hline FeII 2374 & 5300.842 & $<0.008$ \\
\hline FeII 2383 & 5319.380 & $0.011 \pm 0.007$ \\
\hline FeII $2587^{\mathrm{d}}$ & 5774.541 & $0.008 \pm 0.002$ \\
\hline Mg II 2796 & 6242.688 & $0.101 \pm 0.009$ \\
\hline Mg II 2803 & 6258.715 & $0.070 \pm 0.008$ \\
\hline $\operatorname{Mg}$ I $2853^{\mathrm{d}}$ & 6369.071 & $0.005 \pm 0.002$ \\
\hline $\mathrm{S} 28$ & Q0958 + 551 & $z_{\mathrm{abs}}=1.27238$ \\
\hline FeII 2374 & 5395.671 & $<0.007$ \\
\hline FeII $2383^{\mathrm{d}}$ & 5414.540 & $0.009 \pm 0.002$ \\
\hline FeII 2587 & 5877.844 & $<0.007$ \\
\hline FeII $2600^{\mathrm{d}, \mathrm{f}}$ & 5908.573 & $0.017 \pm 0.004$ \\
\hline Mg II 2796 & 6354.366 & $0.081 \pm 0.007$ \\
\hline Mg II 2803 & 6370.679 & $0.056 \pm 0.008$ \\
\hline $\operatorname{Mg}$ I $2853^{\mathrm{d}}$ & 6483.010 & $0.007 \pm 0.002$ \\
\hline S29 & $\mathrm{Q} 0117+212$ & $z_{\mathrm{abs}}=1.32500$ \\
\hline AlıII 1863 & 4331.013 & $0.0162 \pm 0.004$ \\
\hline FeII $2344^{\mathrm{d}}$ & 5450.307 & $0.006 \pm 0.002$ \\
\hline FeII 2374 & 5520.632 & $<0.017$ \\
\hline FeII 2383 & 5539.938 & $0.030 \pm 0.010$ \\
\hline FeII 2587 & 6013.972 & $0.010 \pm 0.005$ \\
\hline FeII 2600 & 6045.412 & $0.026 \pm 0.009$ \\
\hline Mg II 2796 & 6501.530 & $0.291 \pm 0.009$ \\
\hline Mg II 2803 & 6518.221 & $0.180 \pm 0.011$ \\
\hline $\operatorname{Mg}$ I $2853^{\mathrm{d}}$ & 6633.153 & $0.005 \pm 0.002$ \\
\hline
\end{tabular}


TABle 3-Continued

\begin{tabular}{ccc}
\hline \hline Ion/Tran & $\begin{array}{c}\lambda_{\text {abs }}{ }^{\mathrm{a}} \\
{[\AA]}\end{array}$ & $\begin{array}{c}\mathrm{EW}_{\mathrm{r}} \\
{[\AA]}\end{array}$ \\
\hline \multicolumn{1}{c}{ S30 } & Q0117+212 & $z_{\text {abs }}=1.34297$ \\
\hline AlIII 1855 & 4345.600 & $0.031 \pm 0.003$ \\
AlII 1863 & 4364.461 & $0.022 \pm 0.003$ \\
FeII 2344 & 5492.421 & $0.018 \pm 0.005$ \\
FeII 2374 & 5563.289 & $<0.014$ \\
FeII 2383 & 5582.745 & $0.029 \pm 0.004$ \\
FeII 2587 & 6060.441 & $0.012 \pm 0.005$ \\
Mg II 2796 & 6501.530 & $\ldots$ \\
MgII 2803 & 6568.586 & $0.153 \pm 0.008$ \\
MgI 2853 & 6684.406 & $<0.010$ \\
\hline
\end{tabular}

${ }^{\mathrm{a}}$ When multiple components are present, $\lambda_{\mathrm{abs}}$ is $\lambda_{\mathrm{r}}\left(1+z_{\mathrm{abs}}\right)$.

${ }^{\mathrm{b}}$ Compromised by bad pixel (see $\$ 3.2$ ).

${ }^{\mathrm{c}}$ Confused by blending (see $\$ 3.2$ )

${ }^{\mathrm{d}}$ Not a $5 \sigma$ detection, but at least a $>3 \sigma$ detection.

${ }^{\mathrm{e}}$ Compromised by pen mark on the CCD.

${ }^{\mathrm{f}}$ Not captured by the CCD. 
TABLE 4

Limits FOR Undetected TRANSitions

\begin{tabular}{|c|c|c|c|c|}
\hline Ion/Tran & $N_{\text {cov }}$ & $N_{3 \sigma}$ & $\begin{array}{c}W_{\mathrm{r}}^{\min }(3 \sigma) \\
{[\AA]}\end{array}$ & $\begin{array}{c}\left\langle W_{\mathrm{r}}(3 \sigma)\right\rangle \\
{[\AA \AA]}\end{array}$ \\
\hline Alı 2263 & 17 & 0 & 0.003 & 0.008 \\
\hline AlI 2367 & 21 & 0 & 0.003 & 0.008 \\
\hline Ali 3083 & 21 & 0 & 0.003 & 0.006 \\
\hline Ali 3945 & 4 & 0 & 0.005 & 0.007 \\
\hline AlıII 1855 & 3 & 2 & 0.004 & 0.010 \\
\hline AlıII 1863 & 4 & 3 & 0.004 & 0.007 \\
\hline BeII 3131 & 22 & 0 & 0.003 & 0.007 \\
\hline BeII 3132 & 22 & 0 & 0.003 & 0.007 \\
\hline CaI 4228 & 1 & 0 & 0.008 & 0.008 \\
\hline CaII 3935 & 2 & 0 & 0.005 & 0.007 \\
\hline CaII 3970 & 4 & 0 & 0.005 & 0.006 \\
\hline CoII 1941 & 8 & 0 & 0.003 & 0.035 \\
\hline CoII 2012 & 12 & 0 & 0.003 & 0.015 \\
\hline CoII 2026 & 12 & 0 & 0.003 & 0.014 \\
\hline CoII 2059 & 13 & 0 & 0.003 & 0.014 \\
\hline CrII 2056 & 13 & 0 & 0.003 & 0.014 \\
\hline CrII 2062 & 13 & 0 & 0.003 & 0.015 \\
\hline Cr II 2066 & 14 & 0 & 0.003 & 0.014 \\
\hline FeI 2167 & 16 & 0 & 0.003 & 0.010 \\
\hline FeI 2463 & 25 & 0 & 0.003 & 0.008 \\
\hline FeI 2484 & 24 & 0 & 0.003 & 0.008 \\
\hline FeI 2502 & 27 & 0 & 0.003 & 0.007 \\
\hline Fe I 2524 & 26 & 0 & 0.003 & 0.007 \\
\hline FeI 2720 & 29 & 0 & 0.003 & 0.006 \\
\hline FeI 2968 & 23 & 0 & 0.003 & 0.006 \\
\hline FeI 3022 & 22 & 0 & 0.003 & 0.006 \\
\hline FeI 3721 & 7 & 0 & 0.004 & 0.006 \\
\hline FeII 1902 & 6 & 0 & 0.003 & 0.009 \\
\hline FeII 2250 & 16 & 0 & 0.003 & 0.008 \\
\hline FeII 2261 & 17 & 0 & 0.003 & 0.008 \\
\hline FeII 2344 & 20 & 5 & 0.003 & 0.008 \\
\hline FeII 2368 & 21 & 0 & 0.003 & 0.008 \\
\hline FeII 2374 & 21 & 2 & 0.003 & 0.009 \\
\hline FeII 2383 & 22 & 11 & 0.003 & 0.008 \\
\hline FeII 2587 & 29 & 9 & 0.003 & 0.007 \\
\hline FeII 2600 & 27 & 8 & 0.003 & 0.010 \\
\hline
\end{tabular}


TABLE 4-Continued

\begin{tabular}{ccccc}
\hline \hline Ion/Tran & $N_{\text {cov }}$ & $N_{3 \sigma}$ & $\begin{array}{c}W_{\mathrm{r}}^{\min }(3 \sigma) \\
{[\AA]}\end{array}$ & $\begin{array}{c}\left\langle W_{\mathrm{r}}(3 \sigma)\right\rangle \\
{[\AA]}\end{array}$ \\
\hline Mg I 1828 & 2 & 0 & 0.012 & 0.013 \\
Mg I 2026 & 12 & 0 & 0.003 & 0.014 \\
Mg I 2853 & 29 & 7 & 0.003 & 0.006 \\
Mg II 2796 & 29 & 29 & 0.003 & 0.006 \\
Mg II 2803 & 30 & 30 & 0.003 & 0.006 \\
MnII 2577 & 28 & 0 & 0.003 & 0.007 \\
MnII 2594 & 29 & 0 & 0.003 & 0.007 \\
MnII 2606 & 27 & 0 & 0.003 & 0.007 \\
NaI 3303 & 16 & 0 & 0.003 & 0.006 \\
NaI 3304 & 16 & 0 & 0.003 & 0.006 \\
Ni 2312 & 19 & 0 & 0.003 & 0.008 \\
Ni 2321 & 19 & 0 & 0.003 & 0.010 \\
Si 1807 & 2 & 0 & 0.012 & 0.012 \\
SiI 1846 & 3 & 0 & 0.005 & 0.011 \\
SiI 2515 & 27 & 0 & 0.003 & 0.008 \\
SiII 1808 & 2 & 0 & 0.010 & 0.011 \\
TiII 3067 & 22 & 0 & 0.003 & 0.007 \\
TiII 3074 & 21 & 0 & 0.003 & 0.006 \\
TiII 3230 & 20 & 0 & 0.003 & 0.007 \\
TiII 3243 & 17 & 0 & 0.003 & 0.007 \\
TiII 3385 & 13 & 0 & 0.003 & 0.006 \\
ZnII 2026 & 12 & 0 & 0.003 & 0.015 \\
ZnII 2063 & 13 & 0 & 0.003 & 0.015 \\
\hline
\end{tabular}

